\title{
INTERNET NUMBERS
}

\section{STATUS OF THIS MEMO}

This memo is an official status report on the network numbers used in the Internet community. Distribution of this memo is unlimited.

Introduction

The responsibility for the assignment of IP numbers and ASNs has been assumed by Hostmaster at the DDN Network Information Center (NIC). The Hostmaster staff are indebted to Dr. Jon Postel and Ms. Joyce Reynolds of the Information Sciences Institute at the University of Southern California for their ongoing assistance.

This Network Working Group Request for Comments documents the currently assigned network numbers and gateway autonomous systems. This RFC will be updated periodically, and in any case current information can be obtained from Hostmaster.

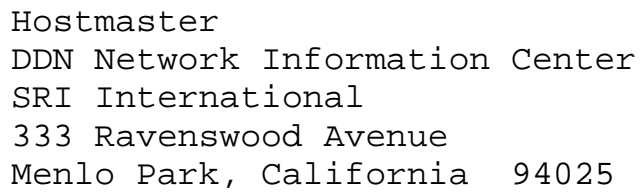


The entries below contain the name and network mailbox of the individuals responsible for each registered network or autonomous system. The bracketed entry, e.g., [nn,iii], at the right hand margin of the page indicates a reference for the listed network or autonomous system, where the number ("nn") cites the document and the letters ("iii") cites the handle of the responsible person. The NIC Handle is a unique identifier that is used in the NIC WHOIS (NICNAME) service. People sometimes change electronic mailboxes. To find out the latest mailbox or phone number of a contact, use the NIC WHOIS/NICNAME server or contact HOSTMASTERQSRI-NIC.ARPA.

The convention used for the documentation of Internet Protocols is to express numbers in decimal and to picture data in "big-endian" order [31]. That is, fields are described left to right, with the most significant octet on the left and the least significant octet on the right.

The order of transmission of the header and data described in this document is resolved to the octet level. Whenever a diagram shows a group of octets, the order of transmission of those octets is the normal order in which they are read in English. For example, in the following diagram the octets are transmitted in the order they are numbered.

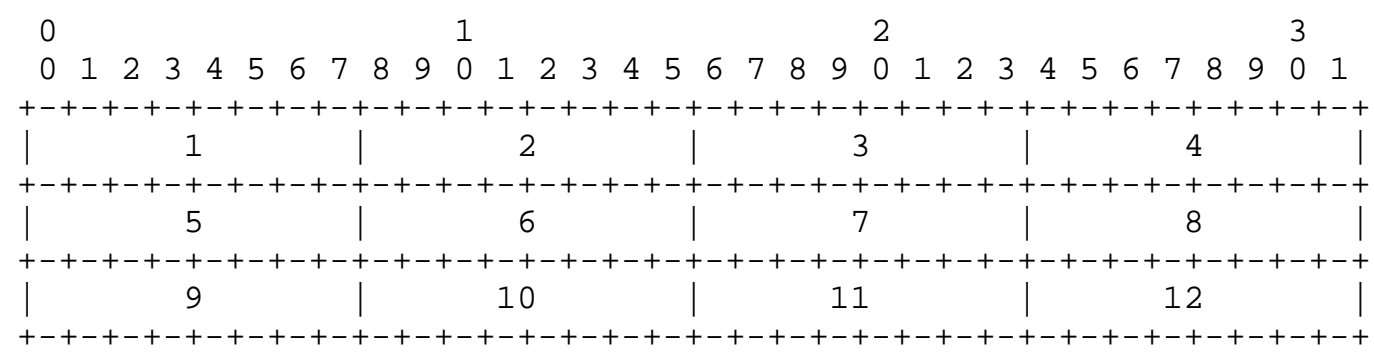

\section{Transmission Order of Bytes}

Whenever an octet represents a numeric quantity the left most bit in the diagram is the high order or most significant bit. That is, the bit labeled 0 is the most significant bit. For example, the following diagram represents the value 170 (decimal).

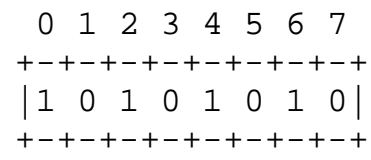

Significance of Bits 
Similarly, whenever a multi-octet field represents a numeric quantity the left most bit of the whole field is the most significant bit. When a multi-octet quantity is transmitted the most significant octet is transmitted first.

\section{NETWORK NUMBERS}

The network numbers listed here are used as internet addresses by the Internet Protocol (IP) [11,21]. The IP uses a 32-bit address field and divides that address into a network part and a "rest" or local address part. The division takes 4 forms or classes.

The first type of address, or class A, has a 7-bit network number and a 24-bit local address. The highest-order bit is set to 0 . This allows 128 class A networks.

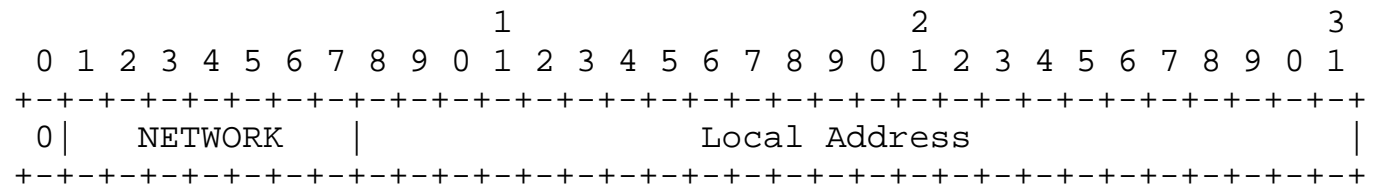

Class A Address

The second type of address, class B, has a 14-bit network number and a 16-bit local address. The two highest-order bits are set to 1-0. This allows 16,384 class B networks.

$\begin{array}{llllllllllllllllll}1 & 2 & & & & \end{array}$

$\begin{array}{llllllllllllllllllllllllllllllll}0 & 1 & 2 & 3 & 4 & 5 & 6 & 7 & 8 & 9 & 0 & 1 & 2 & 3 & 4 & 5 & 6 & 7 & 8 & 9 & 0 & 1 & 2 & 3 & 4 & 5 & 6 & 7 & 8 & 9 & 0 & 1\end{array}$ +-+-+-+-+-+-+-+-+-+-+-+-+-+-+-+-+-+-+-+-+-+-+-+-+-+-+-+-+-+-+-+-+
$\mid 10$

Class B Address

The third type of address, class C, has a 21-bit network number and a 8-bit local address. The three highest-order bits are set to 1-1-0. This allows 2,097,152 class C networks.

1 2

$\begin{array}{llllllllllllllllllllllllllllllll}0 & 1 & 2 & 3 & 4 & 5 & 6 & 7 & 8 & 9 & 0 & 1 & 2 & 3 & 4 & 5 & 6 & 7 & 8 & 9 & 0 & 1 & 2 & 3 & 4 & 5 & 6 & 7 & 8 & 9 & 0 & 1\end{array}$ +-+-+-+-+-+-+-+-+-+-+-+-+-+-+-+-+-+-+-+-+-+-+-+-+-+-+-+-+-+-+-+-+
$\left|\begin{array}{lll}1 & 1 & 0\end{array}\right|$
+-+-+-+-+-+-+-+-+-+-+-+-+-+-+-+-+-+-+-+-+-+-+-+-+-+-+-+-+-+-+-+-+ Class C Address 
The fourth type of address, class D, is used as a multicast address [10]. The four highest-order bits are set to 1-1-1-0.

\section{1}

2

3

$\begin{array}{llllllllllllllllllllllllllllllll}0 & 1 & 2 & 3 & 4 & 5 & 6 & 7 & 8 & 9 & 0 & 1 & 2 & 3 & 4 & 5 & 6 & 7 & 8 & 9 & 0 & 1 & 2 & 3 & 4 & 5 & 6 & 7 & 8 & 9 & 0 & 1\end{array}$

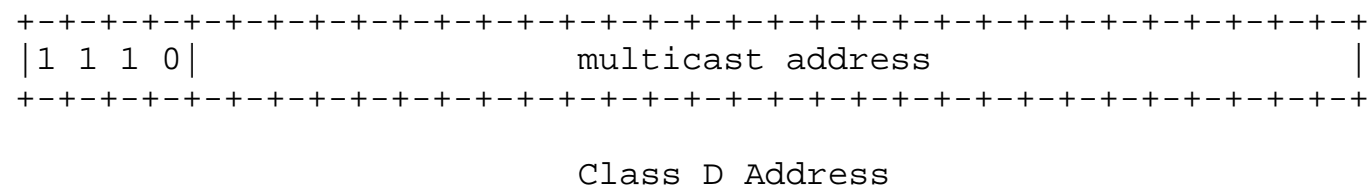

Note: No addresses are allowed with the four highest-order bits set to 1-1-1-1. These addresses, called "class E", are reserved.

One commonly used notation for internet host addresses divides the 32-bit address into four 8-bit fields and specifies the value of each field as a decimal number with the fields separated by periods. This is called the "dotted decimal" notation. For example, the internet address of VENERA.ISI.EDU in dotted decimal is 010.001.000.052, or 10.1 .0 .52 .

The dotted decimal notation will be used in the listing of assigned network numbers. The class A networks will have nnn.rrr.rrr.rrr, the class B networks will have nnn.nnn.rrr.rrr, and the class C networks will have nnn.nnn.nnn.rrr, where nnn represents part or all of a network number and rrr represents part or all of a local address.

There are four catagories of users of Internet Addresses: Research, Defense, Government (Non-Defense), and Commercial. To reflect the allocation of network identifiers among the categories, a onecharacter code is placed to the left of the network number: $\mathrm{R}$ for Research, D for Defense, G for Government, and C for Commercial (see Appendix A for further details on this division of the network identification).

Network numbers are assigned for networks that are connected to the research Internet and operational Internet, and for independent networks that use the IP family protocols (these are usually commercial). These independent networks are marked with an asterisk preceding the number.

The administrators of independent networks must apply separately for permission to interconnect their network with the Internet. Independent networks should not be listed in the working tables of the Internet hosts or gateways.

For various reasons, the assigned numbers of networks are sometimes changed. To ease the transition the old number will be listed for a 


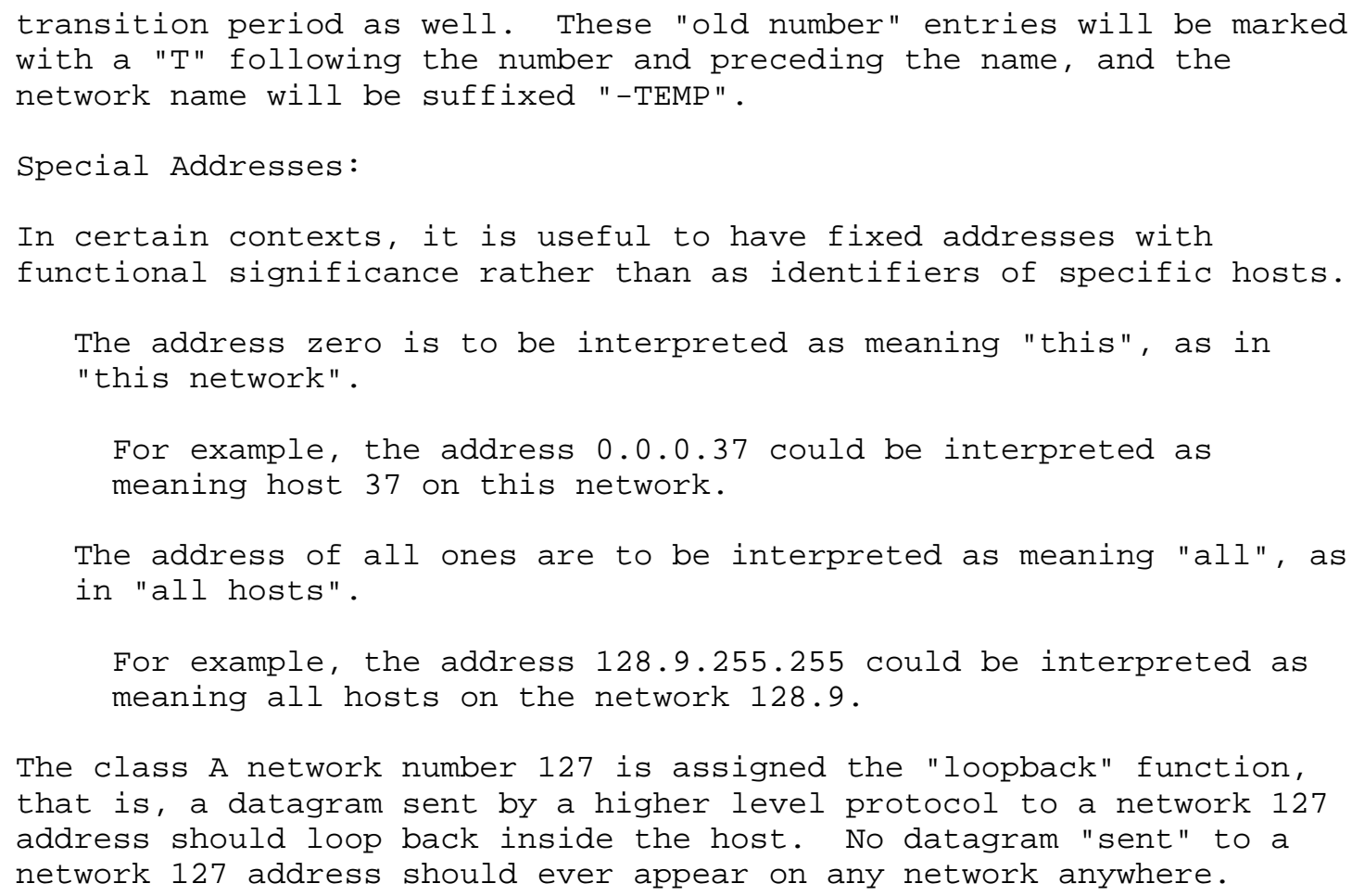


Class A Networks

\begin{tabular}{|c|c|c|c|c|c|}
\hline & Internet Address & Name & Network & & erences \\
\hline & ---------------- & & ------- & & \\
\hline & $000 . \operatorname{rrr} . \operatorname{rrr} \operatorname{srr}$ & & Reserved & & {$[\mathrm{JBP}]$} \\
\hline & 001 .rre.rer.rer-00 & 03.rrr.rre.rrr & Unassigned & & \\
\hline & $004 . r r r . r r r . r r r$ & SATNET & Atlantic Satellite Networ & & \\
\hline & 05.rrr.rrr.rrr & Unassigned & Unassigned & & \\
\hline & $006 . \operatorname{rrr}$.rre.rrr $\mathrm{T}$ & YPG-NET-TEMP & Yuma Proving Grounds & & {$[4, \mathrm{~B}$} \\
\hline & $07 . r r r . r r r . r r r ~ T$ & EDN-TEMP & DCEC EDN & & \\
\hline & 008 .rre.rrr.rer $\mathrm{T}$ & BBN-NET-TEMP & BBN Network & & [ JS \\
\hline & $009 . r r r . r r r . r r r$ & Unassigned & Unassigned & & \\
\hline & 10.rrr.rrr.rrr & ARPANI & ARPANET & & $4, \mathrm{JS} 2$ \\
\hline & $011 . \operatorname{rrr}$.rre.rrr & DODIIS & DOD INTEL INFO SYS & & \\
\hline & $012 \cdot \operatorname{rrr} \cdot \operatorname{rrr} \operatorname{srr}$ & $\mathrm{ATT}$ & ATT, Bell Labs & & \\
\hline & $013 . \operatorname{rrr} \operatorname{srr} \operatorname{srr}$ & XEROX-NET & XEROX Internet & & $39, \mathrm{JN}$ \\
\hline & 014 .rrr.rrr.rrr & $\mathrm{PDN}$ & Public Data Network & & \\
\hline & 015.rrr.rrr.rrr & HP-INTERNET & Hewlett-Packard-Internet & & {$[13,1$} \\
\hline & $016 . \operatorname{rrr}$.rrr.rrr -01 & 17.rrr.rre.rrr & Unassigned & & \\
\hline & 018.rrr.rrr.rrr $\mathrm{T}$ & $\mathrm{MIT}-\mathrm{TEMP}$ & MIT Network & & $31, \mathrm{DD}$ \\
\hline & 019.rrr.rrr.rrr-02 & 20.rrr.rrr.rrr & ssigned & & \\
\hline & $021 . \operatorname{rrr} . \operatorname{rrr} \cdot \operatorname{rrr}$ & $\mathrm{DDN}-\mathrm{R}$ & $\mathrm{VN}$ & & \\
\hline & 022 .rrr.rrr.rrr & DISNET & $\mathrm{T}$ & & \\
\hline & $023 \cdot \operatorname{rrr} \operatorname{srr} \operatorname{trr}$ & $\mathrm{DDN}-\mathrm{TC}$ & TestCell-Network & & \\
\hline & 024.rrr.rrr.rrr & Unassi & signed & & \\
\hline & $025 \cdot \operatorname{rrr} \cdot \operatorname{rrr} \operatorname{rrr}$ & RSRE- & & & \\
\hline & .rre.rrr.rrr & MILNET & $\mathrm{NET}$ & & {$[\mathrm{F}$} \\
\hline & 027 .rre.rer.rer $\mathrm{T}$ & $\mathrm{NOSC}-\mathrm{LCC}$ & NOSC / LCCN & & \\
\hline & $028 . \operatorname{rrr} . \operatorname{rrr} \operatorname{rrr}$ & WIDEB & Wide Band Satellite Net & & {$[\mathrm{C}$} \\
\hline & 029.rrr.rer.rer $\mathrm{T}$ & MILX25 & MILNET X.25 Temp & & \\
\hline & $030 . \operatorname{rrr}$.rre.rrr $\mathrm{T}$ & ARPA & A X.25 Temp & & \\
\hline & .rrr.rrr.rrr & $\mathrm{UCDLA}-\mathrm{NET}$ & A-CATALOG-NET & & {$[\mathrm{C}$} \\
\hline & 2.rrr.rer.rrr & Unassigned & signed & & \\
\hline & .rre.rrr.rrr-03 & 34.rrr.rrr.rrr & signed & & \\
\hline & 035.rrr.rrr.rrr & MERIT & MERIT COMPUTER NETWK & & \\
\hline & 5.rre.rre.rrr $\mathrm{T}$ & SU-NET-T & Stanford University Netwo & & \\
\hline & 037 .rrr.rrr.rrr -03 & 38.rrr.rrr.rrr & Unassigned & & \\
\hline & 039.rrr.rrr.rrr $\mathrm{T}$ & SRINET-TEMP & SRI Local Network & & \\
\hline & $040 . \operatorname{rrr}$.rrr.rrr & Unassigned & Unassigned & & \\
\hline & .rre.rrr.rrr & $\mathrm{BBN}-\mathrm{TEST}-\mathrm{A}$ & $\mathrm{BBN}-\mathrm{GATE}-\mathrm{TEST}-\mathrm{A}$ & & \\
\hline & .rre.rrr.rrr & CAN-INET & Canadian Research Net & & $39, \mathrm{PAP} 4$ \\
\hline & $043 \cdot \operatorname{rrr} \cdot \operatorname{rrr} \operatorname{rrr}$ & Unassigned & Unassigned & & \\
\hline & $044 \cdot \operatorname{rrr} \cdot \operatorname{rrr} \operatorname{srr}$ & AMPRNET & Amateur Radio Experiment & Net & {$[\mathrm{PK} 2$} \\
\hline & $045 . \operatorname{rrr}$.rrr.rrr- & 26.rrr.rrr. & Unassigned & & \\
\hline & 127.rrr.rrr.rrr & & Loopback & & {$[\mathrm{JBF}$} \\
\hline
\end{tabular}


Class B Networks

* Internet Address

- ---------------

R 128.001.rrr.rrr

R 128.002.rrr.rrr

R 128.003.rrr.rrr

R 128.004.rrr.rrr

R 128.005.rrr.rrr

R 128.006.rrr.rrr

R 128.007.rrr.rrr

R 128.008.rrr.rrr

R 128.009.rrr.rrr

R 128.010.rrr.rrr

R 128.011.rrr.rrr

R 128.012.rrr.rrr

D 128.013.rrr.rrr

R 128.014.rrr.rrr

R 128.015.rrr.rrr

R 128.016.rrr.rrr

D 128.017.rrr.rrr

R 128.018.rrr.rrr

D 128.019.rrr.rrr

D 128.020.rrr.rrr

R 128.021.rrr.rrr

R 128.022.rrr.rrr

R 128.023.rrr.rrr

R 128.024.rrr.rrr

D 128.025.rrr.rrr

D 128.026.rrr.rrr

D 128.027.rrr.rrr

D $128.028 . r r r . r r r$

R 128.029.rrr.rrr

R 128.030.rrr.rrr

R 128.031.rrr.rrr

R 128.032.rrr.rrr

R 128.033.rrr.rrr

R 128.034.rrr.rrr

R 128.035.rrr.rrr

R 128.036.rrr.rrr

D 128.037.rrr.rrr

D 128.038.rrr.rrr

R 128.039.rrr.rrr

R 128.040.rrr.rrr

R 128.041.rrr.rrr

R 128.042.rrr.rrr

R 128.043.rrr.rrr
Name

----

BBN-TEST-B

CMU-NET

LBL-CSAM

DCNET

FORDNET

RUTGERS

KRAUTNET

UMDNET

ISI-NET

PURDUE-CS-EN

BBN-CRONUS

SU-NET

MATNET

BBN-SAT-TEST

SINET

UCLNET

MATNET-ALT

SRINET

EDN

BRLNET

$S F-P R-1$

$S F-P R-2$

$\mathrm{BBN}-\mathrm{PR}$

ROCKWELL-PR

BRAGG-PR

$S A C-P R$

DEMO-PR-1

C3-PR-TEMP

MITRE

MIT-NET

MIT-RES

UCB-ETHER

$\mathrm{BBN}-\mathrm{NET}$

NOSC-LCCN

CISLTESTNET1

YALE-NET

YPG-NET

NSWC-NET

NTANET

$\mathrm{UCL}-\mathrm{NET}-\mathrm{A}$

UCL-NET-B

RICE-NET

DRENET
Network

Reserved

BBN-GATE-TEST-B

CMU-Ethernet

LBL-CSAM-RESEARCH

LINKABIT DCNET

FORD DCNET

RUTGERS

KRAUTNET

Univ of Maryland DCNET

USC-ISI Local Network

Purdue CS Ethernet

BBN DOS Project

Stanford University Net

Mobile Access Terminal Net

BBN SATNET Test Net

LLL-S1-NET

University College London

Mobile Access Terminal Alt

SRI Local Network

DCEC EDN

BRLNET

SF-1 Packet Radio Network

SF-2 Packet Radio Network

BBN Packet Radio Network

Rockwell Packet Radio Net

Ft. Bragg Packet Radio Net

SAC Packet Radio Network

Demo-1 Packet Radio Network

Testbed Development PR NET

MITRE Cablenet

MIT Local Network

MIT Research Network

UC Berkeley Ethernet

BBN Network

NOSC / LCCN

Honeywell

YALE NET

Yuma Proving Grounds

NSWC Local Host Net

NDRE-TIU

UCL

UCL

Rice University

Canada REF ARPANET
References

$---------$

[ JBP ]

[RH 6]

[HDW2 ]

[ JS38 ]

[26, DLM1 ]

[26, DLM1 ]

[CLH3 ]

[GB 7 ]

[2 6, DLM1 ]

[CMR ]

$[39, \mathrm{DT} 50]$

[25, PK19]

[LB3]

[ $\mathrm{SHB}$ ]

[SHB]

[RAK12]

$[\mathrm{PK}]$

[ $\mathrm{SHB}$ ]

[ JMR ]

[EC5 ]

[4, MJM2 ]

[ JEM]

[ JEM]

[ JBW1 ]

[EHP ]

[ JEM ]

[VDC1]

[LCS ]

[VDC1]

$[37, \mathrm{TML}]$

[DDC1]

[DDC1]

[RWH5 ]

[ JSG5]

[RH 6]

$[17,18$, JLM2 3 ]

[39, HML1]

[4,BWA ]

[RFH2 ]

[PS27]

[BAW9]

[BAW9]

[39, PGM]

[4, JR1 7] 


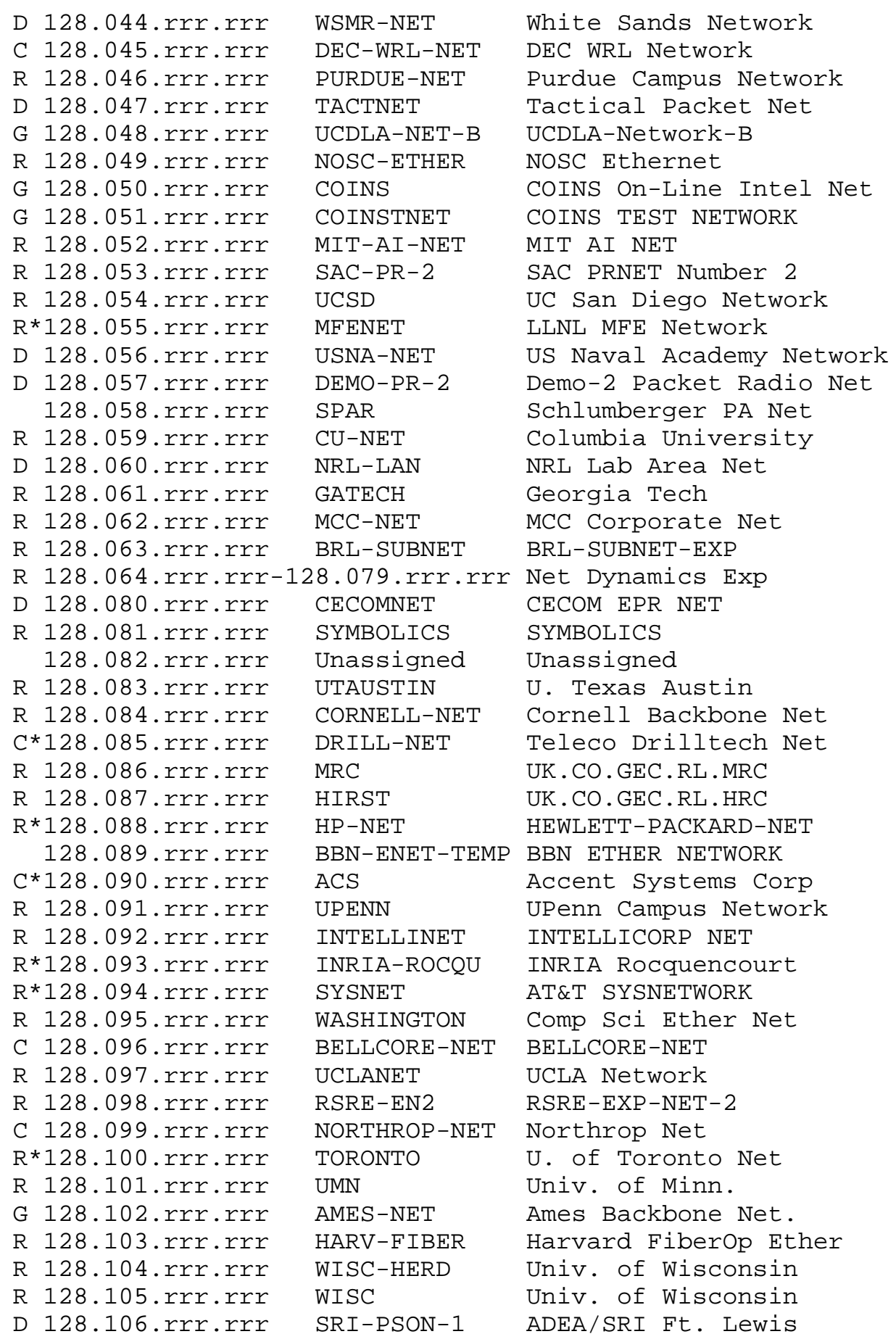

[CAS 1]

[39, RKJ2 ]

[DT50]

$[3, \mathrm{KTP}]$

[4, CL64]

[39, RLB3 ]

[RLS6]

[RLS6]

[39, MDC ]

[VDC1]

[39, GH29]

[36, DRP ]

[TS9]

[LCS ]

[39, SL10]

[39, BC14]

[WF 3 ]

$[39, \mathrm{DD} 11]$

[39, CBD ]

[RBN1]

[ZSU]

[PFS2]

[39, $\mathrm{CH} 2]$

[NIC]

$[39, \mathrm{JBC} 2]$

[39, DK2 ]

[DBJ ]

[RHC3 ]

[RHC3 ]

[AG67]

[39, SGC ]

$[39, \mathrm{ERC} 1]$

[39, IW5 ]

[39, DAVE ]

[MS171]

[EY5]

[39, RA17]

[PK28]

[RBW]

[ JW156]

[39, RSM1 ]

[39, BD55]

[SB12]

[39, MSM1 ]

[39, SB28]

[39, EJN1]

[39, JB188]

[ERK3] 
D 128.107.rrr.rrr

D 128.108.rrr.rrr

R 128.109.rrr.rrr

R 128.110.rrr.rrr

R 128.111.rrr.rrr

R 128.112.rrr.rrr

R 128.113.rrr.rrr

R 128.114.rrr.rrr

R 128.115.rrr.rrr

R 128.116.rrr.rrr

R 128.117.rrr.rrr

R 128.118.rrr.rrr

R 128.119.rrr.rrr

R 128.120.rrr.rrr

R 128.121.rrr.rrr

R 128.122.rrr.rrr

$R * 128.123 . r r r . r r r$

R 128.124.rrr.rrr

R 128.125.rrr.rrr

R 128.126.rrr.rrr

C*128.127.rrr.rrr

R 128.128.rrr.rrr

C*128.129.rrr.rrr

$R * 128.130 . r r r . r r r$

$R * 128.131 . r r r . r r r$

$G * 128.132 . r r r . r r r$

$G * 128.133 . r r r . r r r$

R 128.134.rrr.rrr

R 128.135.rrr.rrr

R 128.136.rrr.rrr

C*128.137.rrr.rrr

R 128.138.rrr.rrr

R 128.139.rrr.rrr

R 128.140.rrr.rrr

$R * 128.141 . r r r . r r r$

$R * 128.142 . r r r . r r r$

$R * 128.143 . r r r . r r r$

$R * 128.144 . r r r . r r r$

R 128.145.rrr.rrr

R 128.146.rrr.rrr

R 128.147.rrr.rrr

R 128.148.rrr.rrr

G 128.149.rrr.rrr

G 128.150.rrr.rrr

R 128.151.rrr.rrr

C 128.152.rrr.rrr

R 128.153.rrr.rrr

G 128.154.rrr.rrr
LEWIS-PRNET1

LEWIS-PRNET2

TUCC-MCNC

$\mathrm{UTAH}-\mathrm{NET}$

UCSB

PRINCETON

RP INET

UCSC

LLL-LABNET

USAN

UCAR

PENN-STATE

UMASS-CS

UCDAVIS

JVNC-NET

NYU-NET

NMSU

T NTA-TEMP

USCNET

SDC-PRC

FTP-SOFTWARE

WHOINET

CGI

TUNET-T

TUNET-F

RADC-LONS

AFSC-LONS

SDN

$\mathrm{U}-\mathrm{CHICAGO}$

TEK-ALLNET

GENNET1

COLORADO

ILAN

EMORY-INET

CERN-ETHER

CERN-TOKEN

VIRGINIA

ARC-CALGARY

NYSERNET

OHIO-STATE

$\mathrm{U}-\mathrm{PGH}-\mathrm{NET}$

BROWN-UNIV

JPL-NET

NSF-LAN

UR-NET

HAC-ENET

CLARKSON

GSFC-NET
ADEA/SRI Ft. Lewis

ADEA/SRI Ft. Lewis

TUCC-MCNC NC Net

UTAH-CAMPUS-NET

U of CA, Santa Barbara

Princeton University

RPI-LOCALNET

U.C. Santa Cruz Net

LLNL Open Labnet

UNIV SATELLITE NET

UNIV CORP ATM RSCH

Penn State Network

UMass COINS Dept LAN

U.C. Davis Network

John von Neumann Ctr Net

NYU Campus Network

$N$ M State Univ

NTARE BF-TO-PDP11

USC Campus Network

SDC Paoli R\&D Center

FTP Software Net

WHOI Campus Net

Carnegie Group

TU Wien Terminal Net

TU Wien File Net

RADC-LONS Net

AFSC-LONS Net

System Dev Net

UNIVERS I TYOFCHICAGO

Teknowledge-Net

Genentech Corp Net

U Colorado Boulder

Israel Academic Net

Emory Internet

DD Main Ethernet

DD Main IBM Token Ring

Univ. of Virginia

Alta Research Calgary

NYSERNET

Ohio state Univ

Univ. Pittsburgh Net

Brown University Net

JPL Central Net

NSF-LAN

Univ. of Rochester

Hughes Aircraft VLSI Net

Clarkson University

GSFC Central Net
[ERK3 ]

[ERK3]

[ JRR14 ]

[ JL15]

[PKH1]

[LRR1]

[MS 9]

$[39$, JHH 8 ]

[BANDY]

[39, BLI ]

[39, BLI ]

[SJS11]

[39, GW4 0 ]

[39, RH5 ]

[SH37 ]

[BJR2 ]

[39, MSP 1]

[TM10 ]

$[39, \mathrm{MAB} 4]$

[39, MS22 ]

[ JLR4 ]

[ARM5 ]

[RA62 ]

[39, GP 56 ]

[39, GP 56 ]

[39, GG43 ]

$[39, \mathrm{GG} 43]$

$[5,6, \mathrm{HC} 2]$

[39, MC17]

[39, TE16]

[39, SM96]

[39, RAJ8 ]

[39, DB35 ]

[39, SA29]

[39, BMS2 ]

[39, BMS2 ]

$[39$, JAJ17 ]

[DK6 6]

[MS 9]

[RSD2]

[SM6]

[MR2 9]

[MSM1]

[FW17]

[TM57]

[PH4 5]

[ JCH17]

[MSM1] 


\begin{tabular}{|c|c|c|c|}
\hline G 128.155.rrr.rrr & LARC-NET & LARC Central Net & [MSM1 ] \\
\hline G 128.156.rrr.rrr & LERC-NET & LERC Central Net & [MSM1 ] \\
\hline $128.157 . \operatorname{rrr} . \operatorname{rrr}$ & $\mathrm{JSC}-\mathrm{NET}$ & JSC Central Net & [MSM1 ] \\
\hline $128.158 . \operatorname{rrr} \operatorname{rrr}$ & MSFC-NET & MSFC Central Net & [MSM1 ] \\
\hline $128.159 . \operatorname{rrr} \operatorname{rrr}$ & $\mathrm{KSC}-\mathrm{NET}$ & KSC Central Net & [MSM1 ] \\
\hline $128.160 . \operatorname{rrr} . r r r$ & NSTL-NET & NSTL Central Net & [MSM1 ] \\
\hline $128.161 . \operatorname{rrr} . \operatorname{rrr}$ & NSN-NET & NASA Science Net & [MSM1 ] \\
\hline $128.162 \cdot \operatorname{rrr} \cdot \operatorname{rrr}$ & CRAY-NET & Cray Research & [DB14] \\
\hline $128.163 . \operatorname{rrr} \operatorname{rrr}$ & UKY & Univ of Kentucky & [GB 43$]$ \\
\hline $128.164 . \operatorname{rrr} . \operatorname{rrr}$ & GWU-GATE & George Washington $\mathrm{U}$. & [TT35 ] \\
\hline G 128.165.rrr.rrr & LANL-INET & LANL Inter-Network & [ JC11] \\
\hline$D * 128.166 . \operatorname{rrr} . \operatorname{rrr}$ & $\mathrm{BAC}-\mathrm{NET}$ & Boeing Aerospace Corp Net & [JJ48 ] \\
\hline R 128.167.rrr.rrr & SURA & SURAnet & [ JH92 ] \\
\hline C $128.168 . \operatorname{rrr}$.rrr & GOLDHILL & Gold-Hill-Computers & [ GM3 4 ] \\
\hline R 128.169.rrr.rrr & UTK & Univ Tenn-Knoxville & [ JDC20 ] \\
\hline R 128.170.rrr.rrr & SDC-CAM & SDC Camarillo R\&D Net & [DSR ] \\
\hline$R * 128.171$.rrr.rrr & HAWAI I & Univ. of Hawaii & [BC32] \\
\hline R 128.172.rrr.rrr & VCU-LAN & VCU-LAN & {$[\mathrm{JN} 40]$} \\
\hline R 128.173.rrr.rrr & $\mathrm{VA}-\mathrm{TECH}$ & Virgina Tech Net & {$[\mathrm{PB} 40]$} \\
\hline R 128.174.rrr.rrr & UIUC-CAMPUS-B & UIUC Campus Network & [PP 14$]$ \\
\hline R 128.175.rrr.rrr & UDELNET & U. of Delaware Network & [DJG2 ] \\
\hline$R * 128.176 . r r r . r r r$ & DMSWWU-ETHER & DMSWWU ETHERNET & [GR26] \\
\hline C*128.177.rrr.rrr & $\mathrm{BLI}-\mathrm{NET}$ & Britton Lee Network & {$[\mathrm{EPA}]$} \\
\hline$R * 128.178 . \operatorname{rrr} \operatorname{rrr}$ & EPF-ETHER1 & Ecublens Campus Net & {$[\mathrm{YXD}]$} \\
\hline $\mathrm{R} * 128.179 . \operatorname{rrr} . \operatorname{rrr}$ & EPF-ETHER2 & Cedres Campus Net & {$[\mathrm{YXD}]$} \\
\hline R 128.180.rrr.rrr & LEHIGH & Lehigh University & {$[39, \operatorname{MM1} 49]$} \\
\hline C*128.181.rrr.rrr & TEKTRONIX & Tektronix Engineering & [ JB218] \\
\hline R 128.182.rrr.rrr & PSCNET & PSC Affiliates Net & [ JTE2 ] \\
\hline R 128.183.rrr.rrr & GSFC & GSEC NASA & [ JB113] \\
\hline $\mathrm{R} * 128.184 . \operatorname{rrr} \operatorname{srr}$ & DEAKINET & Deakinet Univ Net & [ JM303 ] \\
\hline C 128.185.rrr.rrr & PROTEON-NET & Proteon Network & [ JS28 ] \\
\hline R 128.186.rrr.rrr & FSU & Florida State Univ & [ $\mathrm{KMH} 8$ ] \\
\hline$R * 128.187 . \operatorname{rrr} \operatorname{srr}$ & BYU-NET & Brigham Young Net & [ $\mathrm{KCM} 2]$ \\
\hline$R \star 128.188 . r r r . r r r$ & M2CNET & Mass VLSI/CAD Net & [SD1 ] \\
\hline$R * 128.189 . \operatorname{rrr} \operatorname{srr}$ & $\mathrm{BCNET}$ & British Columbia Net & {$[\mathrm{DO} 26]$} \\
\hline G 128.190.rrr.rrr & BELVOIR-G/W & BRADEC Subnet & [DH30] \\
\hline C*128.191.rrr.rrr & NECIS-NET & NEC Info Systems Net & {$[\mathrm{DP} 71]$} \\
\hline R 128.192.rrr.rrr & UGA & UGNET & [EHH4] \\
\hline R 128.193.rrr.rrr & ORST & Oregon State Univ Net & [BA26] \\
\hline R 128.194.rrr.rrr & TAMU-NET & Texas A\&M Univ & [WCE2 ] \\
\hline R 128.195.rrr.rrr & UCIICS-NET & UCI ICS Network & [RAJ3 ] \\
\hline R 128.196.rrr.rrr & UNIV-ARIZ & U of ARIZ Research Net & [ALG4 ] \\
\hline R 128.197.rrr.rrr & $\mathrm{BU}-\mathrm{NET}$ & $\mathrm{BU}-\mathrm{NET}$ & {$[\mathrm{BS} 24]$} \\
\hline R 128.198.rrr.rrr & CU-COLOSPGS & CU-Colorado-Spgs-Net & {$[39, \mathrm{RDG} 12]$} \\
\hline$R * 128.199 . r r r . r r r$ & $\mathrm{STC}$ & STC PLC Company Net & [AM54] \\
\hline $\mathrm{R}$ 128.200.rrr.rrr & $\mathrm{UCI}-\mathrm{NET}$ & UCI Campus Network & [DW96] \\
\hline R 128.201.rrr.rrr & REUNIR & Reseau des universites & [RN25 ] \\
\hline D 128.202 .rrr.rrr & CSOCNET & 2 SW SPACENET LAN & [JJD12 ] \\
\hline
\end{tabular}




\begin{tabular}{|c|c|c|c|}
\hline$R * 128.203 . \operatorname{rrr} . r r r$ & $\mathrm{UB}-\mathrm{INC}$ & Ungermann-Bass Inc & {$[\mathrm{DXC}]$} \\
\hline R 128.204.rrr.rrr & ALBNYNET & U at Albany Net & [BEC1] \\
\hline $\mathrm{R}$ 128.205.rrr.rrr & UBUFFALONET & UNIVOFBUFFALONET & {$[\mathrm{CFD} 4]$} \\
\hline $128.206 . \operatorname{rrr} . r r r$ & Unassigned & Unassigned & [NIC ] \\
\hline$C * 128.207 . \operatorname{rrr} . \operatorname{rrr}$ & BOEING-PSN & Boeing-Puget Sound & {$[39, \mathrm{JSY} 2]$} \\
\hline R 128.208.rrr.rrr & WASH-NSF & WASHINGTON-NSF & {$[39, \mathrm{SH} 47]$} \\
\hline C 128.209.rrr.rrr & NYNEXSTNET & NYNEX Sci and Tech & {$[\mathrm{MC} 65]$} \\
\hline R 128.210.rrr.rrr & PURDUE-CCNET & Purdue Computing Ctr & {$[39, \mathrm{JS} 81]$} \\
\hline $\mathrm{R}$ 128.211.rrr.rrr & PURDUE-CS-CYP & CYPRESS-HUB-PURDUE & {$[\mathrm{DEC} 1]$} \\
\hline$C \star 128.212 . \operatorname{rrr} . r r r$ & ISCNET & ISC Corporate Network & {$[39, \mathrm{DM} 27]$} \\
\hline R 128.213.rrr.rrr & RPICSNET & RPI CSNETWORK & {$[39, \mathrm{MS} 9]$} \\
\hline R 128.214.rrr.rrr & FUNET & Finnish Univ Network & {$[39, \mathrm{JH} 141]$} \\
\hline$C \star 128.215 . r r r . r r r$ & INTEL-NET & INTEL Engineering Network & {$[12, \mathrm{HC} 24]$} \\
\hline $\mathrm{R} 128.216 . \operatorname{rrr} \operatorname{srr}$ & CC-PRNET & CENTCOM Packet Radio Net & {$[39, \mathrm{GIH}]$} \\
\hline $\mathrm{G} * 128.217 . \operatorname{rrr} . \operatorname{rrr}$ & NASA-KSC-OIS & NASA-KSC-OIS & {$[39, \mathrm{GG} 43]$} \\
\hline R 128.218.rrr.rrr & $\mathrm{UCSF}-\mathrm{NET}$ & Univ of Calif, San Fran & {$[39, \mathrm{TF} 6]$} \\
\hline R 128.219.rrr.rrr & ORNL-NETB1 & ORNL Local Area Network & {$[24$, THD $]$} \\
\hline R 128.220.rrr.rrr & JHU & Johns Hopkins Univ & {$[39, \mathrm{MH} 98]$} \\
\hline R 128.221.rrr.rrr & DGPN1 & Data General Priv Net 1 & {$[39, \operatorname{PSS} 1]$} \\
\hline C 128.222.rrr.rrr & DGPN2 & Data General Priv Net 2 & {$[39, \operatorname{PSS} 1]$} \\
\hline R 128.223.rrr.rrr & UONET & Univ of Oregon Network & {$[39, \mathrm{DS} 85]$} \\
\hline$C \star 128.224 . r r r . r r r$ & EP ILOGUE & Epilogue Technology & {$[\mathrm{KA} 4]$} \\
\hline$C * 128.225 . \operatorname{rrr} . \operatorname{rrr}$ & BOEING-EN & Boeing-East Network & {$[39$, JSY3 $]$} \\
\hline R 128.226.rrr.rrr & BINGHAMTON & UNIVATBINGHAMTON & {$[39, \operatorname{RM} 120]$} \\
\hline R 128.227.rrr.rrr & UFNET & Univ of Florida Net & {$[39, \mathrm{AW} 48]$} \\
\hline R 128.228.rrr.rrr & CUNY & City Univ of New York & {$[39, \operatorname{SMP} 2]$} \\
\hline R 128.229.rrr.rrr & ADSNET & Advanced Decision Sys Net & {$[39, \mathrm{MB} 26]$} \\
\hline R 128.230.rrr.rrr & SYR-UNIV-NET & Syracuse Univ Network & {$[39, \mathrm{JW} 47]$} \\
\hline G 128.231.rrr.rrr & $\mathrm{NIH}-\mathrm{NET}$ & Natl Institutes of Health & {$[12, \operatorname{RF} 57]$} \\
\hline$R * 128.232$.rrr.rrr & $\mathrm{CL}-\mathrm{CAM}-\mathrm{AC}-\mathrm{UK}$ & Univ of Cambridge Comp Lab & {$[39, \mathrm{MAJ} 1]$} \\
\hline$R * 128.233 . \operatorname{rrr} . \operatorname{rrr}$ & USASK & Univ of Saskatchewan Net & {$[39, \operatorname{LRC} 7]$} \\
\hline$R * 128.234 . r r r . r r r$ & COS-NET & Cos Network & {$[39, \operatorname{AP} 25]$} \\
\hline R 128.235.rrr.rrr & NJIT & NJIT Network & {$[39, \mathrm{BM} 79]$} \\
\hline D $128.236 . \operatorname{rrr} . r r r$ & USAFA-NET & US Air Force Academy Net & {$[39, \mathrm{GEOFF}]$} \\
\hline R 128.237.rrr.rrr & $\mathrm{CMU}-\mathrm{SEI}-\mathrm{NET}$ & SEI Ethernet & {$[39, \mathrm{PDB} 5]$} \\
\hline R 128.238.rrr.rrr & POLY-U-NET & Polytechnic Univ Net & [39, AMM1 4] \\
\hline R 128.239.rrr.rrr & WM-NET & William and Mary Net & [39, SF 34 ] \\
\hline R 128.240.rrr.rrr & NCL & Newcastle Campus Net & {$[39, \mathrm{AL} 46]$} \\
\hline R 128.241.rrr.rrr & SESQUINET & SESQUINET & {$[\mathrm{GTA}]$} \\
\hline R 128.242.rrr.rrr & MIDNET & Midwest Regional Network & [MM147] \\
\hline$R * 128.243 . r r r . r r r$ & $\mathrm{NOTT}-\mathrm{AC}-\mathrm{UK}$ & Univ of Nottingham Net & {$[39, W A 16]$} \\
\hline D $128.244 . r r r . r r r$ & APL-NET & Applied Physics Lab Net & {$[39, \mathrm{SAK} 3]$} \\
\hline R 128.245.rrr.rrr & $\mathrm{SRA}-\mathrm{CT}-\mathrm{NET}$ & SRA-CONNECTICUT-NET & $5,16, \mathrm{JSS} 4]$ \\
\hline$C \star 128.246 . \operatorname{rrr} . \operatorname{rrr}$ & CGCH-WIRZ & WIRZ Scientific Net & {$[12, \mathrm{HN} 3]$} \\
\hline C 128.247.rrr.rrr & TI & Texas Instruments & [DF71] \\
\hline R 128.248.rrr.rrr & $\mathrm{UIC}-\mathrm{NET}$ & Univ of Illinois-Chicago & {$[39$, EZ3 $]$} \\
\hline R 128.249.rrr.rrr & $\mathrm{TMC}-\mathrm{NET}$ & Texas Medical Center Net & {$[39, \mathrm{SB} 98]$} \\
\hline$R * 128.250 . \operatorname{rrr} . r r r$ & UNIMELB & University of Melbourne & {$[39, \operatorname{CC} 89]$} \\
\hline
\end{tabular}




\begin{tabular}{|c|c|c|c|}
\hline$C * 128.251 . \operatorname{rrr} . \operatorname{rrr}$ & ROCKW-TELEDA & Rockwell-Telecom & {$[39, \mathrm{JCW} 12]$} \\
\hline $\mathrm{R} 128.252 . \operatorname{rrr} \operatorname{srr}$ & WASHINGTON-U & Washington Univ Net & {$[21, \mathrm{DGH} 13]$} \\
\hline $\mathrm{R}$ 128.253.rrr.rrr & $\mathrm{CCS}-\mathrm{NET}$ & Cornell Univ Computer Net & {$[30, \mathrm{DC} 126]$} \\
\hline $\mathrm{R} * 128.254 . \operatorname{rrr} . \operatorname{rrr}$ & FMC-NOD & $\mathrm{FMC}-\mathrm{NOD}$ & {$[39, \mathrm{WCW} 7]$} \\
\hline R 128.255.rrr.rrr & UIOWA & Univ of Iowa & [LT28] \\
\hline $129.000 . \operatorname{rrr} \operatorname{srr}$ & & Reserved & {$[\mathrm{NIC}]$} \\
\hline R 129.001.rrr.rrr & BGSU & Bowling Green State Univ & {$[30, \mathrm{SH} 71]$} \\
\hline R 129.002.rrr.rrr & UMD-BOGON-NET & UMD student Network & {$[39, \mathrm{LAM} 1]$} \\
\hline$R * 129.003 . \operatorname{rrr} . \operatorname{rrr}$ & SUNY-OSWEGO-NET & State Univ NY - Oswego & {$[39, \mathrm{PRT} 2]$} \\
\hline C 129.004.rrr.rrr & TRW & TRW Information Network & {$[39, \mathrm{GGB} 2]$} \\
\hline$R * 129.005 . \operatorname{rrr} . \operatorname{rrr}$ & HGCNET & HARTFORDGRADCTRNET & {$[38, \mathrm{AG} 61]$} \\
\hline G $129.006 . \operatorname{rrr}$.rrr & NBS & NBS Network & {$[39, \mathrm{CWH} 3]$} \\
\hline R 129.007.rrr.rrr & $\mathrm{UH}-\mathrm{NET}$ & Univ. of Houston Network & {$[39, \mathrm{JH} 155]$} \\
\hline$R * 129.008 . \operatorname{rrr} . \operatorname{rrr}$ & CSUFRESNO & CSUFresno CSci Net & {$[39, \operatorname{RP} 88]$} \\
\hline$C * 129.009 . \operatorname{rrr} . \operatorname{rrr}$ & CHRYSLER-NET & CHRYSLER-INTERNET & {$[30, \operatorname{RER} 20]$} \\
\hline$R * 129.010 . r r r . r r r$ & NORTHEASTERN & Northeastern Network & {$[39, \mathrm{CJ} 38]$} \\
\hline $\mathrm{R} * 129.011 . \operatorname{rrr} \operatorname{rrr}$ & LEEDS & Leeds University Network & {$[39, \mathrm{AJC}]$} \\
\hline$R * 129.012 . \operatorname{rrr} . \operatorname{rrr}$ & $\mathrm{UKC}$ & UKC Campus Net & {$[39, \operatorname{SL} 55]$} \\
\hline$R * 129.013 . \operatorname{rrr} \operatorname{srr}$ & LINK & Karlsruhe Network & {$[39, \operatorname{MR} 78]$} \\
\hline$C * 129.014 . \operatorname{rrr} . \operatorname{rrr}$ & SBINY & Salomon Brothers Inc. & {$[39, \mathrm{BC} 72]$} \\
\hline R 129.015.rrr.rrr & UOKNOR & Univ of Okla, Norman & [ JW136] \\
\hline$R * 129.016 . r r r . r r r$ & $\mathrm{CTH}-\mathrm{NET}$ & Chalmers University & [GL41] \\
\hline $\mathrm{R} * 129.017 . \operatorname{rrr} . \operatorname{rrr}$ & SSED-NET & Honeywell-SSED-NET & [DM147] \\
\hline$C \star 129.018 . \operatorname{rrr} . \operatorname{rrr}$ & $\mathrm{NEXT}-\mathrm{NET}$ & NeXT Inc. Network & {$[39, \mathrm{PFK}]$} \\
\hline R 129.019.rrr.rrr & WESTNET & Western Regional Net & {$[39, \mathrm{DCMW}]$} \\
\hline $\mathrm{R} * 129.020 . \operatorname{rrr} . \operatorname{rrr}$ & VERDUR & Universite de Rennes & [RN25 ] \\
\hline$R * 129.021 . r r r . r r r$ & RIT & Rochester Inst of Tech & {$[39, \mathrm{CF} 35]$} \\
\hline$R * 129.022 . \operatorname{rrr} . \operatorname{rrr}$ & CWRUNET & CWRU Campus Network & {$[39, \mathrm{JAG} 3]$} \\
\hline $\mathrm{R} 129.023 . \operatorname{rrr}$.rrr & SDIO-INTERNET & SDIO Wide Area Internet & {$[39, \mathrm{KDZ}]$} \\
\hline $\mathrm{R} 129.024 . \operatorname{rrr} \operatorname{srr}$ & UNMNET & Univ. of New Mexico Network & $\mathrm{k} \quad[39, \mathrm{KDZ}]$ \\
\hline R 129.025.rrr.rrr & DREXEL & DREXEL UNIVERSITY & {$[39, \operatorname{RR} 97]$} \\
\hline$R * 129.026 . r r r . r r r$ & GMD-DE & GMD Net & {$[39, \mathrm{PM} 72]$} \\
\hline$R * 129.027 . \operatorname{rrr} . \operatorname{rrr}$ & WEDGE-NET & Wedge Computer Net & {$[\mathrm{DTH}]$} \\
\hline$C \star 129.028 . \operatorname{rrr} . r r r$ & ETA-LAN & ETA-LAN st. Paul & {$[2, \mathrm{DMK} 16]$} \\
\hline D $129.029 . \operatorname{rrr}$.rrr & WESTPOINTNET & U.S. Army West Point & {$[39, \mathrm{BAT} 4]$} \\
\hline C $129.030 . \operatorname{rrr}$.rrr & HONEYWELL & HONEYWELL INC NETWORK & {$[39, \mathrm{DB} 97]$} \\
\hline$R * 129.031 . \operatorname{rrr} . \operatorname{rrr}$ & ICNET & IC Campus Net & {$[39, \mathrm{LM} 88]$} \\
\hline R 129.032.rrr.rrr & TEMP LE & Temple Univ Network & 39, TES16] \\
\hline $\mathrm{R} 129.033$. rrr.rrr-1 & 129.042 .rrr.rrr & IBM Research Network & [MT1] \\
\hline R 129.043.rrr.rrr & NCI-FCRF & Frederick Cancer Net & {$[39, \mathrm{WLB} 5]$} \\
\hline$C * 129.044 . \operatorname{rrr} . \operatorname{rrr}$ & NYTEL1095NET & NYTEL1095NET & {$[39, \operatorname{HT} 12]$} \\
\hline$C * 129.045 . \operatorname{rrr} . \operatorname{rrr}$ & NYTELNOCNET1 & NYTELNOCNET1 & {$[39, \mathrm{JO5} 4]$} \\
\hline C $129.046 . \operatorname{rrr}$.rrr & QUALNET & QUALCOMM Ethernet & {$[39, \mathrm{TM} 37]$} \\
\hline$C * 129.047 . \operatorname{rrr} . \operatorname{rrr}$ & SYTEK-INC & Sytek Corporation & [AB90] \\
\hline D $129.048 . r r r . r r r$ & WPAFB-CDS-GW & WPAFB-CDS-GATEWAY & {$[39, \mathrm{CMC} 6]$} \\
\hline $.049 . \operatorname{rrr} . \operatorname{rrr}-1$ & $191.254 . \operatorname{rrr} . \operatorname{rrr}$ & Unassigned & {$[\mathrm{NIC}]$} \\
\hline $191.255 . \operatorname{rrr} . \operatorname{rrr}$ & & Reserved & {$[\mathrm{JBP}]$} \\
\hline
\end{tabular}


Class C Networks

\begin{tabular}{|c|c|c|c|c|}
\hline \multirow[t]{3}{*}{ * } & \multirow{2}{*}{ Internet Address } & Name & Network & References \\
\hline & & ---- & ------- & ---------- \\
\hline & $192.000 .000 . \operatorname{rrr}$ & & Reserved & {$[\mathrm{JBP}]$} \\
\hline \multicolumn{2}{|r|}{ R 192.000.001.rrr } & $\mathrm{BBN}-\mathrm{TEST}-\mathrm{C}$ & $\mathrm{BBN}-\mathrm{GATE}-\mathrm{TEST}-\mathrm{C}$ & [ RH 6 ] \\
\hline \multicolumn{2}{|r|}{$R * 192.000 .002 . \operatorname{rrr}$} & TEST & TEST & {$[\mathrm{JBP}]$} \\
\hline \multicolumn{3}{|c|}{$192.000 .003 . r r r-192.000 .255 . r r r$} & Unassigned & {$[\mathrm{NIC}]$} \\
\hline \multicolumn{3}{|c|}{ R 192.001.000.rrr-192.001.004.rrr } & BBN local networks & {$[\mathrm{SGC}]$} \\
\hline $\mathrm{R}$ & $192.001 .005 . r r r$ & $\mathrm{BBN}-\mathrm{ENET} 2$ & $\mathrm{BBN}-\mathrm{ENET} 2$ & {$[\mathrm{SGC}]$} \\
\hline \multicolumn{3}{|c|}{$192.001 .006 . \operatorname{rrr}$} & BBN local network & {$[\mathrm{SGC}]$} \\
\hline $\mathrm{R}$ & \multirow{2}{*}{\multicolumn{2}{|c|}{$\begin{array}{l}192.001 .00 \% . r r r \\
192.001 .008 . r r r\end{array}$}} & $\mathrm{BBN}-\mathrm{ENET}$ & {$[\mathrm{SGC}]$} \\
\hline $\mathrm{R}$ & & & BBN local network & {$[\mathrm{SGC}]$} \\
\hline $\mathrm{R}$ & \multicolumn{2}{|l|}{$192.001 .009 . r r r$} & BBN-ENET3 & {$[\mathrm{SGC}]$} \\
\hline $\mathrm{R}$ & \multicolumn{2}{|l|}{$192.001 .010 . \operatorname{rrr}$} & $\mathrm{BBN}-\mathrm{NETR}$ & {$[\mathrm{SGC}]$} \\
\hline $\mathrm{R}$ & \multicolumn{2}{|c|}{$192.001 .011 . r r r \quad B B N-S P C-E N E T$} & $\mathrm{BBN}-\mathrm{SPC}-\mathrm{ENET}$ & {$[\mathrm{SGC}]$} \\
\hline $\mathrm{R}$ & \multicolumn{2}{|c|}{$192.001 .012 . \operatorname{rrr}-192.003 .255 . \operatorname{rrr}$} & BBN local networks & {$[\mathrm{SGC}]$} \\
\hline \multicolumn{3}{|c|}{$R * 192.004 .000 . r r r-192.004 .255 . \operatorname{rrr}$} & BELLCORE-NET & {$[39, \mathrm{PK} 28]$} \\
\hline $\mathrm{R}$ & $192.005 .001 . r r r$ & CISLHYPERNET & Honeywell & [ JLM23 ] \\
\hline \multicolumn{2}{|r|}{$R * 192.005 .002 . \operatorname{rrr}$} & $\mathrm{UF}-\mathrm{NET}-\mathrm{A}$ & UF-CIS Dept Ether & [AW48 ] \\
\hline $\mathrm{C}$ & $192.005 .003 . \operatorname{rrr}$ & $\mathrm{HP}-\mathrm{DESIGN}-\mathrm{AID}$ & S HP Design Aids & [AG67] \\
\hline \multicolumn{2}{|r|}{$192.005 .004 . \operatorname{rrr}$} & $\mathrm{HP}-\mathrm{TCG}-\mathrm{UNIX}$ & Hewlett Packard TCG Unix & {$[\mathrm{AG} 67]$} \\
\hline $\mathrm{R}$ & $192.005 .005 . \operatorname{rrr}$ & DEC-MRNET & DEC Marlboro Ethernet & {$[39, \mathrm{JM} 60]$} \\
\hline $\mathrm{R}$ & $192.005 .006 . \operatorname{rrr}$ & DEC-MRRAD & DEC Marlboro Developmt & {$[39, \mathrm{JM} 60]$} \\
\hline $\mathrm{R}$ & $192.005 .007 . r r r$ & $\mathrm{CIT}-\mathrm{CS}-\mathrm{NET}$ & Caltech-CS-Net & {$[41, \mathrm{DSW}]$} \\
\hline $\mathrm{R}$ & $192.005 .008 . \operatorname{rrr}$ & MACOMNET & MACOM Network & [SB90] \\
\hline $\mathrm{R}$ & $192.005 .009 . \operatorname{rrr}$ & AERONET & Aerospace Labnet & {$[1, \mathrm{LCN}]$} \\
\hline $\mathrm{R}$ & $192.005 .010 . \operatorname{rrr}$ & ECLNET & USC-ECL-CAMPUS-NET & [MAB 4 ] \\
\hline $\mathrm{R}$ & $192.005 .011 . \operatorname{rrr}$ & CSS-RING & SEISMIC-RESEARCH-NET & [RR2 ] \\
\hline $\mathrm{R}$ & $192.005 .012 . \operatorname{rrr}$ & $\mathrm{UTAH}-\mathrm{NET}-\mathrm{C}$ & UTAH-COMPUTER-SCIENCE-NET & [GW22 ] \\
\hline $\mathrm{R}$ & $192.005 .013 . \operatorname{rrr}$ & GSWDNET & Compion Network & {$[39$, FAS ] } \\
\hline $\mathrm{R}$ & $192.005 .014 . \operatorname{rrr}$ & RAND-NET & RAND Network & {$[39, \mathrm{JDG}]$} \\
\hline $\mathrm{R}$ & $192.005 .015 . \operatorname{rrr} \mathrm{T}$ & NYU-NET-TEMP & NYU Network & {$[\mathrm{EF} 5]$} \\
\hline $\mathrm{R}$ & $192.005 .016 . \operatorname{rrr}$ & LANLLAND & Los Alamos Dev LAN & {$[39, \mathrm{JC} 11]$} \\
\hline $\mathrm{R}$ & $192.005 .017 . \operatorname{rrr}$ & NRL-NET & Naval Research Lab & {$[\mathrm{AP}]$} \\
\hline $\mathrm{R}$ & $192.005 .018 . \operatorname{rrr}$ & IPTO-NET & ARPA-IPTO Office Net & [ JS283] \\
\hline $\mathrm{R}$ & $192.005 .019 . \operatorname{rrr}$ & UCIICS & UCI-ICS Res Net & [MTR ] \\
\hline $\mathrm{R}$ & $192.005 .020 . \operatorname{rrr}$ & CISLTTYNET & Honeywell & [ JLM23 ] \\
\hline D & $192.005 .021 . \operatorname{rrr}$ & BRLNET1 & BRLNET1 & {$[4, \mathrm{MJM} 2]$} \\
\hline $\mathrm{D}$ & $192.005 .022 . \operatorname{rrr}$ & BRLNET2 & BRLNET2 & {$[4, \mathrm{MJM} 2]$} \\
\hline $\mathrm{D}$ & $192.005 .023 . \operatorname{rrr}$ & BRLNET3 & BRLNET3 & {$[4, \mathrm{MJM} 2]$} \\
\hline $\mathrm{D}$ & $192.005 .024 . \operatorname{rrr}$ & BRLNET 4 & BRLNET 4 & {$[4, \mathrm{MJM} 2]$} \\
\hline $\mathrm{D}$ & $192.005 .025 . \operatorname{rrr}$ & BRLNET5 & BRLNET5 & {$[4, \mathrm{MJM} 2]$} \\
\hline $\mathrm{D}$ & $192.005 .026 . \operatorname{rrr}$ & $\mathrm{NSRDCOA}-\mathrm{NET}$ & NSRDC Office Auto Net & [RWT2 ] \\
\hline $\mathrm{D}$ & $192.005 .027 . \operatorname{rrr}$ & DTNSRDC-NET & DTNSRDC-NET & [RWT2 ] \\
\hline $\mathrm{R}$ & $192.005 .028 . \operatorname{rrr}$ & RSRE-NULL & RSRE-NULL & [ RNM1 ] \\
\hline $\mathrm{R}$ & $192.005 .029 . \operatorname{rrr}$ & $\mathrm{RSRE}-\mathrm{ACC}$ & $\mathrm{RSRE}-\mathrm{ACC}$ & [ RNM1 ] \\
\hline $\mathrm{R}$ & $192.005 .030 . \operatorname{rrr}$ & $\mathrm{RSRE}-\mathrm{PR}$ & $\mathrm{RSRE}-\mathrm{PR}$ & [ RNM1 ] \\
\hline
\end{tabular}




\begin{tabular}{|c|c|c|c|c|}
\hline & $\star 192.005 .031 . \operatorname{rrr}$ & SIEMENS-NET & Siemens Research Network & [PN23] \\
\hline & $192.005 .032 . \operatorname{rrr}$ & CISLTESTNET2 & Honeywell & {$[17,18$, JLM23 $]$} \\
\hline $\mathrm{R}$ & $192.005 .033 . \operatorname{rrr}$ & CISLTESTNET3 & Honeywell & {$[17,18$, JLM23 $]$} \\
\hline $\mathrm{R}$ & $192.005 .034 . \operatorname{rrr}$ & CISLTESTNET4 & Honeywell & {$[17,18$, JLM23 $]$} \\
\hline $\mathrm{R}$ & $192.005 .035 . \operatorname{rrr}$ & RIACS & USRA & {$[39, \mathrm{WPJ}]$} \\
\hline $\mathrm{R}$ & $92.005 .036 . r r r$ & CORNELL-CS & CORNELL CS Research & {$[39, \mathrm{DK} 2]$} \\
\hline $\mathrm{R}$ & $92.005 .037 . \operatorname{rrr}$ & $\mathrm{UR}-\mathrm{CS}-\mathrm{NET}$ & $\mathrm{U}$ of $\mathrm{R} \mathrm{CS} 3 \mathrm{Mb}$ Net & {$[39, \mathrm{LB} 16]$} \\
\hline $\mathrm{R}$ & $92.005 .038 . \operatorname{rrr}$ & SRI-C3ETHER & SRI-AITAD C3ETHERNET & {$[39, \mathrm{VDC} 1]$} \\
\hline $\mathrm{R}$ & $92.005 .039 . \operatorname{rrr}$ & UDEL-EECIS & Udel EECIS LAN & {$[39, \mathrm{DJG} 2]$} \\
\hline $\mathrm{R}$ & $92.005 .040 . \operatorname{rrr}$ & PUCC-NET-A & PURDUE Comp Cntr Net & [ JRS 8 ] \\
\hline D & $92.005 .041 . r r r$ & WISLAN & WIS Research LAN & {$[39, \mathrm{JRM} 1]$} \\
\hline $\mathrm{D}$ & $92.005 .042 . \operatorname{rrr}$ & HYPER-1ISG & AFDSC Hypernet & [MCA1] \\
\hline $\mathrm{R}$ & $92.005 .043 . r r r$ & CUCSNET & Columbia CS Net & {$[39, \mathrm{BC} 14]$} \\
\hline $\mathrm{R}$ & $192.005 .044 . \operatorname{rrr}$ & FARBER-PC-NET & Farber PC Network & {$[\mathrm{DJF}]$} \\
\hline $\mathrm{R}$ & $92.005 .045 . \operatorname{rrr}$ & AIDS-NET & AI\&DS Network & {$[39, \mathrm{KFD}]$} \\
\hline $\mathrm{R}$ & $192.005 .046 . \operatorname{rrr}$ & NTA-RING & NDRE-RING & [PS27] \\
\hline $\mathrm{R}$ & $92.005 .047 . \operatorname{rrr}$ & NSRDC & NSRDC & [RWT2 ] \\
\hline $\mathrm{R}$ & $92.005 .048 . \operatorname{rrr}$ & PURDUE-CS-NET & Purdue CS ProNET & {$[\mathrm{DT} 50]$} \\
\hline & $192.005 .049 . \operatorname{rrr}$ & Unassigned & Unassigned & {$[\mathrm{NIC}]$} \\
\hline $\mathrm{R}$ & $192.005 .050 . \operatorname{rrr}$ & $\mathrm{CTH}-\mathrm{CS}-\mathrm{NET}$ & Chalmers CSN Net & {$[39, \mathrm{UB} 3]$} \\
\hline $\mathrm{R}$ & $192.005 .051 . \operatorname{rrr}$ & THEORYNET & Cornell Theory Center & {$[39, \mathrm{AB} 13]$} \\
\hline $\mathrm{R}$ & $192.005 .052 . \operatorname{rrr}$ & NLM-ETHER & NLM-LHNCBC-ETHERNET & {$[\mathrm{JA} 1]$} \\
\hline $\mathrm{R}$ & $192.005 .053 . \operatorname{rrr}$ & UR-CS-ETHER & $\mathrm{U}$ of $\mathrm{R}$ CS $10 \mathrm{Mb}$ Net & {$[39, \mathrm{LB} 16]$} \\
\hline $\mathrm{R}$ & $192.005 .054 . \operatorname{rrr}$ & $\mathrm{AERO}-\mathrm{A} 6$ & Aerospace & {$[1, \mathrm{LCN}]$} \\
\hline $\mathrm{R}$ & $92.005 .055 . \operatorname{rrr}$ & UCLA-CECS & UCLA-CECS Network & {$[39, \mathrm{RBW}]$} \\
\hline C & $192.005 .056 . \operatorname{rrr}$ & TARTAN-NET & Tartan Labs & [ED38 ] \\
\hline $\mathrm{R}$ & $192.005 .057 . \operatorname{rrr}$ & UDEL-CC & UDEL Comp Center & {$[39, \operatorname{RR} 18]$} \\
\hline $\mathrm{R}$ & $192.005 .058 . \operatorname{rrr}$ & CSNET-PDN & CSNET X.25 Network & {$[22, \mathrm{RDR} 4]$} \\
\hline & *192.005.059.rrr & INRIA-SM90 & Inria GIP SM-90 & [MS171] \\
\hline & $\star 192.005 .060 . \operatorname{rrr}$ & SM90-X1 & Inria SM-90 exp. 1 & [MS171] \\
\hline & *192.005.061.rrr & SM90-X2 & Inria SM-90 exp. 2 & [MS171] \\
\hline & *192.005.062.rrr & LITP-SM90 & LITP SM-90 & [MS171] \\
\hline & $192.005 .063 . \operatorname{rrr}$ & ENCORE & Encore-Marlboro & {$[$ IRN $]$} \\
\hline & $192.005 .064 . \operatorname{rrr}$ & AMES-NAS-NET & NASA ARC NAS LAN & {$[39, \mathrm{MF} 31]$} \\
\hline & $192.005 .065 . \operatorname{rrr}$ & NPRDC-Ether & NPRDC TRCF Ethernet & {$[\mathrm{LRB}]$} \\
\hline $\mathrm{R}$ & $192.005 .066 . \operatorname{rrr}$ & HARV-NET & Harvard Comp Sci Net & [SB28] \\
\hline & $192.005 .067 . \operatorname{rrr}$ & CECOM-ETHER & CECOM ADDCOMPE ETHER & {$[39, \mathrm{GIH}]$} \\
\hline & $192.005 .068 . \operatorname{rrr}$ & AERO-130 & AEROSPACE-130 & {$[\mathrm{LCN}]$} \\
\hline $\mathrm{R}$ & $192.005 .069 . \operatorname{rrr}$ & UIUC-NET & Univ of IL at Urbana & {$[39, \mathrm{AKC}]$} \\
\hline G & $192.005 .070 . \operatorname{rrr}$ & CELAN & COINS Exper. LAN & [MMM2 5 ] \\
\hline & $192.005 .071 . \operatorname{rrr}$ & SAC-ETHER & SAC C3 Ethernet & {$[39, \mathrm{VDC} 1]$} \\
\hline & *192.005.072.rrr & U CHICAGO & U Chicago & {$[\mathrm{MC} 17]$} \\
\hline & $192.005 .073 . \operatorname{rrr}$ & UOFCHICAGO & U Chicago & [MC1 7] \\
\hline & *192.005.074.rrr-19 & $92.005 .087 . \operatorname{rrr}$ & U Chicago & [MC17] \\
\hline & $192.005 .088 . \operatorname{rrr}$ & YALE-EE-NET & YALE-EE-NET & {$[39, A G 22]$} \\
\hline $\mathrm{R}$ & $192.005 .089 . \operatorname{rrr}$ & HARV-APPOLLO & Harvard University & {$[2, \mathrm{SB} 28]$} \\
\hline $\mathrm{R}$ & $192.005 .090 . \operatorname{rrr}$ & HARV-ETHER & Harvard CS Ethernet & {$[\mathrm{SB} 28]$} \\
\hline & $192.005 .091 . \operatorname{rrr}$ & PURDUE-ECN1 & Purdue ECN & {$[10,20, \mathrm{GG} 11]$} \\
\hline
\end{tabular}




\begin{tabular}{|c|c|c|c|}
\hline R 192.005.092.rrr & BRAGG-ETHER & SRI Bragg Ether & {$[39, \mathrm{GIH}]$} \\
\hline R 192.005.093.rrr & SRI-DEMO & SRI Ether Demo & {$[39, \mathrm{GIH}]$} \\
\hline$R \star 192.005 .094 . \operatorname{rrr}$ & SDCRDCF $-10 \mathrm{MB}$ & SDC R\&D primary net & {$[39, \mathrm{DJV} 1]$} \\
\hline$R * 192.005 .095 . \operatorname{rrr}$ & $S D C R D C F-3 M B$ & SDC R\&D old net & {$[39, \mathrm{DJV} 1]$} \\
\hline$R * 192.005 .096 . \operatorname{rrr}$ & $\mathrm{UBC}-\mathrm{CS}-\mathrm{NET}$ & UBC Comp Sci Net & {$[39, \mathrm{~PB} 67]$} \\
\hline$R * 192.005 .097 . \operatorname{rrr}$ & $\mathrm{UCLA}-\mathrm{CS}-\mathrm{LNI}$ & UCLA CS LNI Network & {$[\mathrm{RBW}]$} \\
\hline$R * 192.005 .098 . \operatorname{rrr}$ & UCLA-P IC & UCLA PIC Network & {$[39, \mathrm{RBW}]$} \\
\hline R 192.005.099.rrr & SPACENET & S-1 Workstation Net. & {$[39, \operatorname{TW} 51]$} \\
\hline$R * 192.005 .100 . \operatorname{rrr}$ & $\mathrm{HCSC}-\mathrm{NET}$ & Honeywell CSC Net & {$[39, \mathrm{TRG} 4]$} \\
\hline R 192.005.101.rrr & PUCC-NET-B & Purdue Gateway Network & [ JRS 8 ] \\
\hline R 192.005.102.rrr & PUCC-RHF-NET & PUCC RHF Based Net & [ JRS 8 ] \\
\hline$C \star 192.005 .103 . \operatorname{rrr}$ & TYM-NTD-NET & Tymnet NTD Ethernet & {$[\mathrm{SMF} 5]$} \\
\hline R 192.005.104.rrr & THINK-INET & Thinking Machines & {$[39, \mathrm{BJN} 1]$} \\
\hline R 192.005.105.rrr & CCA-POND & CCA Ethernet1 (POND) & {$[42, \mathrm{AL} 6]$} \\
\hline$C * 192.005 .106 . \operatorname{rrr}$ & BITSTREAM & Bitstream Type Foundry & {$[39, \mathrm{PGA} 1]$} \\
\hline$R * 192.005 .107 . \operatorname{rrr}$ & PASC-ETHER & IBM PASC Ethernet & {$[39, \mathrm{GAL} 5]$} \\
\hline$R * 192.005 .108 . \operatorname{rrr}$ & $\mathrm{PASC}-\mathrm{BB}$ & IBM PASC Broadband & {$[20$, GAL 5$]$} \\
\hline$R * 192.005 .109 . \operatorname{rrr}$ & $\mathrm{CWR}-\mathrm{JCC}-\mathrm{T}$ & ARJCC TOPS-20 NET & {$[39$, JAG3 $]$} \\
\hline$R * 192.005 .110 . \operatorname{rrr}$ & CWR-JCC-L & ARJCC LOCAL NET & {$[39, \mathrm{JAG} 3]$} \\
\hline *192.005.111.rrr & CWR-QUAD & Campus QUAD NET & {$[39$, JAG3 $]$} \\
\hline$R * 192.005 .112 . \operatorname{rrr}$ & CWR-CAISR & CAISR LOCAL NET & {$[39, \mathrm{JAG} 3]$} \\
\hline$R * 192.005 .113 . \operatorname{rrr}$ & CWR-CES & CES LOCAL NET & [ JAG3 ] \\
\hline$C * 192.005 .114 . \operatorname{rrr}$ & I2-RING-1 & INTERMETRICS PRONET & {$[39, \mathrm{NH} 2]$} \\
\hline$C \star 192.005 .115 . \operatorname{rrr}$ & I2-ETHER-1 & INTERMETRICS ETHER & {$[39, \mathrm{NH} 2]$} \\
\hline R 192.005.116.rrr & BRAGGNET-1 & BRAGG / ADDCOMPE & {$[39, \mathrm{BG} 25]$} \\
\hline R 192.005.117.rrr & BRAGGNET-2 & BRAGG / ADDCOMPE & {$[39, \mathrm{BG} 25]$} \\
\hline R 192.005.118.rrr & BRAGGNET-3 & BRAGG / ADDCOMPE & {$[39, \mathrm{BG} 25]$} \\
\hline R 192.005.119.rrr & BRAGGNET-4 & BRAGG / ADDCOMPE & {$[39, \mathrm{BG} 25]$} \\
\hline R 192.005.120.rrr & BRAGGNET-5 & BRAGG / ADDCOMPE & {$[39, B G 25]$} \\
\hline R 192.005.121.rrr & BRAGGNET- 6 & BRAGG / ADDCOMPE & {$[39, \mathrm{BG} 25]$} \\
\hline R 192.005.122.rrr & BRAGGNET-7 & BRAGG / ADDCOMPE & {$[39, \mathrm{BG} 25]$} \\
\hline R 192.005.123.rrr & BRAGGNET-8 & BRAGG / ADDCOMPE & {$[39, \mathrm{BG} 25]$} \\
\hline R 192.005.124.rrr & BRAGGNET-9 & BRAGG / ADDCOMPE & {$[39, \mathrm{BG} 25]$} \\
\hline R 192.005.125.rrr & BRAGGNET-10 & BRAGG / ADDCOMPE & {$[39, \mathrm{BG} 25]$} \\
\hline R 192.005.126.rrr & BRAGGNET-11 & BRAGG / ADDCOMPE & {$[39, \mathrm{BG} 25]$} \\
\hline R 192.005.127.rrr & BRAGGNET-12 & BRAGG / ADDCOMPE & {$[39, \mathrm{BG} 25]$} \\
\hline R 192.005.128.rrr & BRAGGNET-13 & BRAGG / ADDCOMPE & {$[39, \mathrm{BG} 25]$} \\
\hline R 192.005.129.rrr & BRAGGNET-14 & BRAGG / ADDCOMPE & {$[39, \mathrm{BG} 25]$} \\
\hline R 192.005.130.rrr & BRAGGNET-15 & BRAGG / ADDCOMPE & {$[39, \mathrm{BG} 25]$} \\
\hline R 192.005.131.rrr & BRAGGNET-1 6 & BRAGG / ADDCOMPE & {$[39, \mathrm{BG} 25]$} \\
\hline R 192.005.132.rrr & BRAGGNET-17 & BRAGG / ADDCOMPE & {$[39, B G 25]$} \\
\hline$R * 192.005 .133 . \operatorname{rrr}$ & PERCEPT-AI & Perceptronics & {$[\mathrm{KC} 8]$} \\
\hline$C \star 192.005 .134 . \operatorname{rrr}$ & I2-ETHER-2 & Intermetrics & {$[39, \mathrm{NH} 2]$} \\
\hline R 192.005.135.rrr & LL-SPEECH-NET & LL Speech Net & {$[39, \mathrm{RH} 60]$} \\
\hline R 192.005.136.rrr & LL43-LEX-BACK & Lincoln G43-LEX-BACK & {$[39, \mathrm{BC} 65]$} \\
\hline R 192.005.137.rrr & LL43-LEX-SUNA & Lincoln G43-LEX-SUNA & {$[39, \mathrm{BC} 65]$} \\
\hline R 192.005.138.rrr & LL43-LEX-SUNB & Lincoln G43-LEX-SUNB & {$[39, \mathrm{BC} 65]$} \\
\hline R 192.005.139.rrr & LL43-LEX-APO & Lincoln G43-LEX-APO & {$[39, \mathrm{BC} 65]$} \\
\hline
\end{tabular}




\begin{tabular}{|c|c|c|}
\hline R 192.005.140.rrr & LL43-TB-BACK & Lincoln G43-TB-BACK \\
\hline R 192.005.141.rrr & LL43-TB-APO & Lincoln G43-TB-APO \\
\hline$R * 192.005 .142 . \operatorname{rrr}$ & CCVR & CCVR Network \\
\hline R 192.005.143.rrr & NWU & NORTHWESTERN \\
\hline R 192.005.144.rrr & $\mathrm{CRE}-\mathrm{NET}$ & CANADA-CRC-ETHERNET \\
\hline R 192.005.145.rrr & ECRC-SL & ECRC-SL Net \\
\hline R 192.005.146.rrr & $\mathrm{CPW}-\mathrm{PSC}$ & Pittsburgh SC Center \\
\hline R 192.005.147.rrr & ALV-ETHER & MMDAALVVAX \\
\hline R 192.005.148.rrr & DISE & Dist Sys Eval Envir \\
\hline R 192.005.149.rrr & RDL-ETHER & $\mathrm{RDL}$ \\
\hline$G * 192.005 .150 . \operatorname{rrr}$ & $\mathrm{SP}-\mathrm{ACE}-\mathrm{NET}$ & Sperry space Sys Net \\
\hline R 192.005.151.rrr & PENN-STATE-1 & Penn State Network \\
\hline R 192.005.152.rrr & PENN-STATE-2 & Penn State Network \\
\hline R 192.005.153.rrr & PENN-STATE-3 & Penn State Network \\
\hline R 192.005.154.rrr & PENN-STATE-4 & Penn State Network \\
\hline R 192.005.155.rrr & PENN-STATE-5 & Penn State Network \\
\hline R 192.005.156.rrr & PENN-STATE-6 & Penn State Network \\
\hline R 192.005.157.rrr & PENN-STATE-7 & Penn State Network \\
\hline R 192.005.158.rrr & PENN-STATE-8 & Penn State Network \\
\hline R 192.005.159.rrr & PENN-STATE-9 & Penn State Network \\
\hline R 192.005.160.rrr & PENN-STATE-10 & Penn State Network \\
\hline R 192.005.161.rrr & PENN-STATE-11 & Penn State Network \\
\hline R 192.005.162.rrr & PENN-STATE-12 & Penn State Network \\
\hline$C \star 192.005 .163 . \operatorname{rrr}$ & I2-SPDNET-1 & I2 SPD Ethernet \\
\hline C $192.005 .164 . \operatorname{rrr}$ & GTEECN & GTE Eng Net \\
\hline R 192.005.165.rrr & $\mathrm{SDC}-\mathrm{CAM}-1$ & SDC Camarillo R\&D Net \\
\hline$R * 192.005 .166 . \operatorname{rrr}$ & CRC-WDC-NET & CRC Washington DC \\
\hline R 192.005.167.rrr & $\mathrm{MCC}-\mathrm{AI}-\mathrm{NET}$ & MCC AI Subnet \\
\hline R 192.005.168.rrr & $\mathrm{MCC}-\mathrm{CAD} 2-\mathrm{NET}$ & MCC CAD2 Subnet \\
\hline R 192.005.169.rrr & $\mathrm{MCC}-\mathrm{PKG}-\mathrm{NET}$ & MCC PKG Subnet \\
\hline G 192.005.170.rrr & ANLNET1 & Argonne Network \\
\hline G 192.005.171.rrr & ANLNET2 & Argonne Network \\
\hline G $192.005 .172 . \operatorname{rrr}$ & ANLNET3 & Argonne Network \\
\hline G 192.005.173.rrr & ANLNET 4 & Argonne Network \\
\hline G 192.005.174.rrr & ANLNET5 & Argonne Network \\
\hline G 192.005.175.rrr & ANLNET 6 & Argonne Network \\
\hline G 192.005.176.rrr & ANLNET7 & Argonne Network \\
\hline G 192.005.177.rrr & ANLNET 8 & Argonne Network \\
\hline G 192.005.178.rrr & ANLNET 9 & Argonne Network \\
\hline G 192.005.179.rrr & ANLNET10 & Argonne Network \\
\hline G 192.005.180.rrr & ANLNET11 & Argonne Network \\
\hline G 192.005.181.rrr & ANLNET12 & Argonne Network \\
\hline G 192.005.182.rrr & ANLNET13 & Argonne Network \\
\hline G 192.005.183.rrr & ANLNET14 & Argonne Network \\
\hline G 192.005.184.rrr & ANLNET15 & Argonne Network \\
\hline G 192.005.185.rrr & ANLNET1 6 & Argonne Network \\
\hline G 192.005.186.rrr & ANLNET17 & Argonne Network \\
\hline G 192.005.187.rrr & ANLNET18 & Argonne Network \\
\hline
\end{tabular}

R 192.005.140.rrr

R $192.005 .144 . r r$

R 192.005.145.rrr

R 192.005.146.rrr

R 192.005.147.rrr

$192.005 .148 . r r$

R 192.005.151.rrr

R 192.005.152.rrr

192.005.153.rr

R 192.005.157.r

R 192.005.158.rrr

R 192.005.159.rrr

R 192.005.160.rr

R 192.005.161.rrr

$192.005 .162 . r r r$

$R * 192.005 .166 . r r r$

R 192.005.167.rrr

R 192.005.168.rrr

$192.005 .169 . r r$

192.005.170.rr

G 192.005.176.rrr

G 192.005.177.rrr

G 192.005.178.rrr

G 192.005.179.rrr

G 192.005.180.rrr

G 192.005.181.rrr

G 192.005.182.rrr

G 192.005.185.rrr

G 192.005.187.rrr

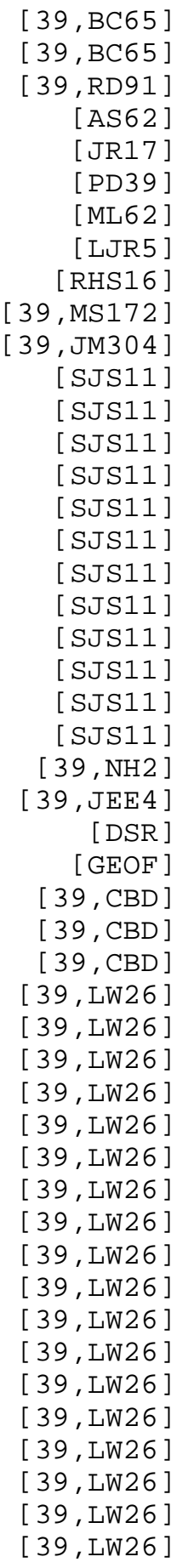

[Page 16] 


\begin{tabular}{|c|c|c|c|}
\hline G 192.005.188.rrr & ANLNET1 9 & Argonne Network & {$[39, \mathrm{LW} 26]$} \\
\hline G 192.005.189.rrr & ANLNET20 & Argonne Network & {$[39, \mathrm{LW} 26]$} \\
\hline G 192.005.190.rrr & ANLNET21 & Argonne Network & {$[39, \mathrm{LW} 26]$} \\
\hline $192.005 .191 . \operatorname{rrr}$ & ANLNET22 & Argonne Network & {$[39, \mathrm{LW} 26]$} \\
\hline $192.005 .192 . \operatorname{rrr}$ & ANLNET23 & Argonne Network & {$[39, \mathrm{LW} 26]$} \\
\hline G 192.005.193.rrr & ANLNET2 4 & Argonne Network & {$[39$, LW2 6] } \\
\hline G 192.005.194.rrr & ANLNET2 5 & Argonne Network & {$[39, \mathrm{LW} 26]$} \\
\hline G 192.005.195.rrr & ANLNET2 6 & Argonne Network & {$[39, \mathrm{LW} 26]$} \\
\hline G 192.005.196.rrr & ANLNET27 & Argonne Network & {$[39, \mathrm{LW} 26]$} \\
\hline G 192.005.197.rrr & ANLNET28 & Argonne Network & {$[39$, LW2 6] } \\
\hline G 192.005.198.rrr & ANLNET29 & Argonne Network & {$[39, \mathrm{LW} 26]$} \\
\hline G 192.005.199.rrr & ANLNET30 & Argonne Network & {$[39, \mathrm{LW} 26]$} \\
\hline G 192.005.200.rrr & ANLNET31 & Argonne Network & {$[39, \mathrm{LW} 26]$} \\
\hline G 192.005.201.rrr & ANLNET32 & Argonne Network & {$[39, \mathrm{LW} 26]$} \\
\hline R 192.005.202.rrr & FMC-CEL & FMC-CEL Host Net & {$[39, \mathrm{KW} 2]$} \\
\hline$R * 192.005 .203 . r r r$ & OKSTATE-CS & Okla. St. CS Network & {$[39, \mathrm{MV} 24]$} \\
\hline R 192.005.204.rrr & SKL-ENET & Canada_SKL_ethernet & {$[\mathrm{JR} 17]$} \\
\hline$R * 192.005 .205 . \operatorname{rrr}$ & ARC-CALGARY & Alta Research Calgary & [DK66] \\
\hline R 192.005.206.rrr & BU-MATHNET & BU-MATHNET & [BS24] \\
\hline R 192.005.207.rrr & BU-CHEMNET & BU-CHEMNET & [BS24] \\
\hline R 192.005.208.rrr & BU-CLANNET & BU-CLANNET & [BS24] \\
\hline D $192.005 .209 . \operatorname{rrr}$ & SSDF-CDCNET & CDC-DDN-DEVELOPMENT & [RE22 ] \\
\hline G 192.005.210.rrr & ECSNET & Embedded Comp Sys Net & {$[\mathrm{CAL} 7]$} \\
\hline R 192.005.211.rrr & INTEL-IWARP & Intel iWarp Net & {$[39, \mathrm{BT} 5]$} \\
\hline R 192.005.212.rrr T & EMORY-INET 4 & Emory Internet 4 & [SA29] \\
\hline R 192.005.213.rrr & HARRIS & Harris-GSSNet & [DAT 4] \\
\hline C 192.005.214.rrr & DECUACNET & Decuac Network & {$[39, \mathrm{FMA} 1]$} \\
\hline R 192.005.215.rrr & MASONNET & GMU Network & {$[39$, TH15] } \\
\hline$R * 192.005 .216 . \operatorname{rrr}$ & $\mathrm{NTT}-\mathrm{NET}$ & NTT Research Lab Net & {$[39, \mathrm{YS} 10]$} \\
\hline R 192.005.217.rrr & YALE-ZOO-NET & Yale Apollo Ed Net & [HML1] \\
\hline R 192.005.218.rrr & ARINC-GW-NET & Yale Apollo Ed Net & {$[\mathrm{YN}]$} \\
\hline R 192.005.219.rrr & CLEMSON & Clemson Univ Comp Center & [DB28 ] \\
\hline C $192.005 .220 . \operatorname{rrr}$ & SCCNET & SPACECOM IP Network & {$[39, \mathrm{MJO} 4]$} \\
\hline$C \star 192.005 .221 . \operatorname{rrr}$ & CSC-LONS & CSC-LONS Network & {$[39, \mathrm{GG} 43]$} \\
\hline$C \star 192.005 .222 . \operatorname{rrr}$ & CSC-OIS & CSC-OIS Network & {$[39, \mathrm{GG} 43]$} \\
\hline$R * 192.005 .223 . \operatorname{rrr}$ & HWELL-RE & HWELL-RESD-ENGRG & {$[39, \mathrm{PP} 36]$} \\
\hline$D * 192.005 .224 . \operatorname{rrr}$ & $\mathrm{HAIC}-\mathrm{NET}$ & Hughes AI Center Net & {$[39, \mathrm{DMK} 18]$} \\
\hline$C \star 192.005 .225 . \operatorname{rrr}-19$ & $92.005 .236 . \operatorname{rrr}$ & GE CALMA BLOCK & {$[39, \mathrm{TR} 38]$} \\
\hline$C \star 192.005 .237 . \operatorname{rrr}$ & PRIME-AI & Prime AI CAD/CAM & {$[22, \mathrm{NSE}]$} \\
\hline$C \star 192.005 .238 . \operatorname{rrr}$ & PALLAD IAN-1 & Palladian-IN1 & [CSTACY] \\
\hline$C \star 192.005 .239 . \operatorname{rrr}$ & PALLADIAN-2 & Palladian-RING & [CSTACY] \\
\hline$C * 192.005 .240 . \operatorname{rrr}$ & PALLADIAN-3 & Palladian-IN2 & [CSTACY] \\
\hline R 192.005.241.rrr & USC-CYPRESS & USC Cypress Network & {$[9, \mathrm{DE} 6]$} \\
\hline$C \star 192.005 .242 . \operatorname{rrr}$ & $\mathrm{MOT}-\mathrm{ASIC}$ & Motorola Chandler LAN & [ GW4 9] \\
\hline$C \star 192.005 .243 . \operatorname{rrr}$ & MOT-MESA & Motorola Mesa LAN & [GW4 9] \\
\hline$C \star 192.005 .244 . \operatorname{rrr}$ & MOT-DOVER & Motorola Dover LAN & [GW4 9 ] \\
\hline$C * 192.005 .245 . \operatorname{rrr}$ & MOT-PRICE & Motorola Prince Road LAN & [GW49 ] \\
\hline$C \star 192.005 .246 . \operatorname{rrr}$ & $\mathrm{MOT}-\mathrm{PICO}$ & Motorola Pico LAN & [GW4 9] \\
\hline
\end{tabular}




\begin{tabular}{|c|c|c|c|}
\hline$C * 192.005 .247 . \operatorname{rrr}$ & $\mathrm{MOT}-52 \mathrm{ND}$ & Motorola Semi MIS LAN & [ GW4 9 ] \\
\hline$C * 192.005 .248 . \operatorname{rrr}$ & MOT-AUSTIN & Motorola Austin LAN & [GW49] \\
\hline$C \star 192.005 .249 . \operatorname{rrr}$ & MOT-OAKHILL & Motorola Oakhill LAN & [GW49 ] \\
\hline$C * 192.005 .250 . \operatorname{rrr}$ & MOT-TELAVIV & Motorola Tel Aviv LAN & [GW4 9] \\
\hline$C * 192.005 .251 . \operatorname{rrr}$ & MOT-GENEVA & Motorola Geneva LAN & [GW4 9] \\
\hline$C \star 192.005 .252 . \operatorname{rrr}$ & MOT-TOKYO & Motorola Tokyo LAN & [ GW4 9 ] \\
\hline$\star 192.005 .253 . \operatorname{rrr}$ & MOT-HONGKONG & Motorola Hongkong LAN & [ GW4 9] \\
\hline$R * 192.005 .254 . \operatorname{rrr}$ & ANSA & ANSA Project & {$[39, \mathrm{DO} 27]$} \\
\hline $192.005 .255 . \operatorname{rrr}$ & Unassigned & Unassigned & {$[\mathrm{NIC}]$} \\
\hline$C * 192.006 .000 . \operatorname{rrr}-1$ & $192.006 .255 . \operatorname{rrr}$ & Hewlett Packard & {$[A G 67]$} \\
\hline$C \star 192.007 .000 . \operatorname{rrr}-1$ & $192.007 .255 . \operatorname{rrr}$ & Computer Consoles, Inc. & [RA11] \\
\hline$C \star 192.008 .000 . \operatorname{rrr}-1$ & $192.008 .255 . \operatorname{rrr}$ & Spartacus Incorporated & [F JK2 ] \\
\hline$C * 192.009 .000 . \operatorname{rrr}-1$ & $192.009 .255 . \operatorname{rrr}$ & SUN Microsystems, Inc. & [BN4] \\
\hline$C \star 192.010 .000 . r r r-1$ & $192.010 .040 . \operatorname{rrr}$ & Symbolics, Inc. & [ $\mathrm{CH} 2]$ \\
\hline R 192.010.041.rrr $\mathrm{T}$ & I SCRC-ETHERNET & SCRC ETHERNET & {$[39, \mathrm{CH} 2]$} \\
\hline$C \star 192.010 .042 . \operatorname{rrr}-1$ & $192.010 .255 . \operatorname{rrr}$ & Symbolics, Inc. & {$[\mathrm{CH} 2]$} \\
\hline$C * 192.011 .000 . \operatorname{rrr}-1$ & $192.011 .255 . \operatorname{rrr}$ & ATT, Bell Labs & [MH 82 ] \\
\hline R 192.012.000.rrr & YALE-SUN-NET & YALE-SUN-NET & [ LFO ] \\
\hline $192.012 .001 . \operatorname{rrr}$ & Unassigned & Unassigned & {$[\mathrm{NIC}]$} \\
\hline $192.012 .002 . \operatorname{rrr}$ & Unassigned & Unassigned & {$[\mathrm{NIC}]$} \\
\hline$C \star 192.012 .003 . r r r$ & FLAIR & Fairchild AI Lab Net & {$[39, \operatorname{AMS1}]$} \\
\hline$C \star 192.012 .004 . \operatorname{rrr}$ & $\mathrm{SCG}-\mathrm{NET}$ & Hughes SCG Net & {$[40, \mathrm{MKP} 2]$} \\
\hline R 192.012.005.rrr & AIC-LISPMS & SRI-AIC-LispMachNet & {$[39, \mathrm{PM} 4]$} \\
\hline R 192.012.006.rrr & $\mathrm{NPS}-\mathrm{C} 2$ & $\mathrm{NPS}-\mathrm{C} 2$ & {$[39$, AW9 $]$} \\
\hline R 192.012.007.rrr & I NYU-CS-ETHER & NYU CompSci Ethernet & {$[39, \mathrm{LOU}]$} \\
\hline D $192.012 .008 . r r r$ & PICANET 1 & Picatinny Arsenal LAN1 & {$[39, \operatorname{RFD} 1]$} \\
\hline R 192.012.009.rrr T & I CADRE-NET & Decision Systems Lab & {$[\mathrm{SM} 6]$} \\
\hline R 192.012.010.rrr & CORNELL-ENG & Cornell-Engineering & {$[39, \mathrm{DK} 2]$} \\
\hline R 192.012.011.rrr & $\mathrm{MIT}-\mathrm{TEST}$ & MIT Gateway TEST NET & {$[39, \mathrm{NC} 3]$} \\
\hline G 192.012.012.rrr & NBS & NBS Network & [ JCN2 ] \\
\hline R 192.012.013.rrr & JHU-NET1 & JHU-NET1 & {$[39, \mathrm{MO} 14]$} \\
\hline R 192.012.014.rrr & JHU-NET2 & JHU-NET2 & {$[39, \mathrm{MO} 14]$} \\
\hline R 192.012.015.rrr & BROOKNET & BNL Brooknet III & {$[39, \mathrm{GC}]$} \\
\hline R 192.012.016.rrr & PRMNET & SRI-SURAN-EN & {$[39, \mathrm{BP} 17]$} \\
\hline G 192.012.017.rrr & LLL-TIS-NET & LLL-TIS-NET & {$[39,40, \mathrm{NAL}]$} \\
\hline R 192.012.018.rrr & CIT-CS-10NET & Caltech $10 \mathrm{Meg}$ EtherNet & {$[41, \mathrm{AD} 22]$} \\
\hline R 192.012.019.rrr & $\mathrm{CIT}-\mathrm{NET}$ & Caltech Campus Net & {$[41, \mathrm{AD} 22]$} \\
\hline R 192.012.020.rrr & CIT-SUN-NET & Caltech Sun Net & {$[41, \mathrm{AD} 22]$} \\
\hline R 192.012.021.rrr & CIT-PHYSCOMP & Caltech Phys Comp Net & {$[41, \mathrm{AD} 22]$} \\
\hline R 192.012.022.rrr & UTCSRES & UTCS Net Research & {$[39, \mathrm{JBC} 2]$} \\
\hline R 192.012.023.rrr & UTCSTTY & UTCS TTY Kludgenet & {$[39, \mathrm{JBC} 2]$} \\
\hline R 192.012.025.rrr & CSS-GRAMINAE & CSS Workstation Net & {$[19, \mathrm{RR} 2]$} \\
\hline R 192.012.026.rrr & NOSC-NETR & Net-R Testbed at BBN & {$[34, \mathrm{CP} 10]$} \\
\hline R 192.012.027.rrr & UR-LASER & UR Laser Energetics & {$[39, \mathrm{WL} 31]$} \\
\hline$R * 192.012 .028 . \operatorname{rrr}$ & RIACS-X-NET & RIACS-Experimental-Net & [DG28] \\
\hline D 192.012.029.rrr & RF-EVANS & ADDCOMPE DC3 LAN1 & {$[39, \mathrm{MB} 31]$} \\
\hline D $192.012 .030 . \operatorname{rrr}$ & $\mathrm{RF}-\mathrm{HEX}-\mathrm{A}$ & ADDCOMPE DC3 LAN2 & {$[39, \mathrm{MB} 31]$} \\
\hline D $192.012 .031 . \operatorname{rrr}$ & USNA-ENET & USNA Engineering Net & {$[39, \operatorname{TS} 9]$} \\
\hline
\end{tabular}




\begin{tabular}{|c|c|c|}
\hline$R * 192.012 .032 . \operatorname{rrr}$ & CMU-VINEYARD & CMU File Cluster Net \\
\hline R 192.012.033.rrr & SRI-CSL-NET & SRI-CSL $10 \mathrm{MB}$ Ethernet \\
\hline$C \star 192.012 .034 . \operatorname{rrr}-1 \mathrm{~s}$ & $92.012 .043 . \operatorname{rrr}$ & Schlumberger PA Net \\
\hline $\mathrm{R} 192.012 .044 . \operatorname{rrr} \mathrm{T}$ & NRTC-NET & Northrop Research Net \\
\hline $192.012 .045 . \operatorname{rrr}$ & $\mathrm{ACC}-\mathrm{SB}-\mathrm{IMP}-\mathrm{NET}$ & I ACC Santa Barbara IMP \\
\hline $192.012 .046 . \operatorname{rrr}$ & $\mathrm{ACC}-\mathrm{SB}-\mathrm{ETHER}$ & ACC Santa Barbara Ethernet \\
\hline R 192.012.047.rrr & UMN-UCC-NET & Univ. of Minnesota \\
\hline G 192.012.048.rrr & AMES-ED-EXPNET & [ Code ED Exp. Net. \\
\hline $192.012 .049 . \operatorname{rrr}$ & AMES-ED-NET & Code ED IP Net \\
\hline $192.012 .050 . \operatorname{rrr}$ & AMES-DB-NET & Ames DBridge Net \\
\hline R 192.012.051.rrr & THINK-CHAOS & TMC Chaos \\
\hline$R * 192.012 .052 . \operatorname{rrr}$ & NEURO-NET & NEURO-NET \\
\hline$R * 192.012 .053 . \operatorname{rrr}$ & $\mathrm{PU}-\mathrm{LCA}$ & Princeton U. LCA \\
\hline R 192.012.054.rrr & AERO-A3 & Aerospace \\
\hline $192.012 .055 . \operatorname{rrr}$ & HAZ-LPR-BETA & Hazeltine LPR Net \\
\hline R 192.012.056.rrr & $\mathrm{UTAH}-\mathrm{AP}-\mathrm{NET}$ & Utah-Appolo-Ring-Net \\
\hline R 192.012.057.rrr & $\mathrm{MCC}-\mathrm{CAD}-\mathrm{NET}$ & MCC CAD Subnet \\
\hline R 192.012.058.rrr & $\mathrm{MCC}-\mathrm{PP}-\mathrm{NET}$ & MCC AI Subnet \\
\hline R 192.012.059.rrr & $\mathrm{MCC}-\mathrm{DB}-\mathrm{NET}$ & MCC DB Subnet \\
\hline $192.012 .060 . \operatorname{rrr}$ & $\mathrm{MCC}-\mathrm{HI}-\mathrm{NET}$ & MCC HI Subnet \\
\hline R 192.012.061.rrr & $\mathrm{MCC}-\mathrm{SW}-\mathrm{NET}$ & MCC SW Subnet \\
\hline R 192.012.062.rrr & DREA-ENET & DREA Lispm \& Vaxen \\
\hline R 192.012.063.rrr & CYPRESS & CYPRESS Serial Net \\
\hline $192.012 .064 . \operatorname{rrr}$ & LOGNET & Logistics Net GW \\
\hline $192.012 .065 . \operatorname{rrr}$ & HELNET1 & HELNET1 \\
\hline D 192.012.066.rrr & HELNET2 & HELNET2 \\
\hline $192.012 .067 . \operatorname{rrr}$ & HELNET3 & HELNET3 \\
\hline G $192.012 .068 . \operatorname{rrr}$ & ORNL-MSRNET & ORNL Local Area Net \\
\hline R 192.012.069.rrr & $\mathrm{UA}-\mathrm{CS}-\mathrm{NET}$ & UNIV. OF ARIZ-CS DEPT \\
\hline $192.012 .070 . \operatorname{rrr}$ & NPRDC-IPD & NPRDC-IPD REMOTE ETHERNET \\
\hline R 192.012.071.rrr & NPRDC-ISG & NPRDC-ISG REMOTE ETHERNET \\
\hline R 192.012.072.rrr & ULCC & $\mathrm{UK} \cdot \mathrm{AC} \cdot \mathrm{ULCC}$ \\
\hline R 192.012.073.rrr & BTRL & UK.CO.BT-RESEARCH-LABS \\
\hline$R * 192.012 .074 . \operatorname{rrr}$ & APPLE-ETHER & APPLE COMPUTER ETHER \\
\hline$R * 192.012 .075 . \operatorname{rrr}$ & PASC-RING & IBM PASC TOKEN RING \\
\hline$R * 192.012 .076 . \operatorname{rrr}$ & $\mathrm{UQ}-\mathrm{NET}$ & UNIV. OF QLD NETWORK \\
\hline$C \star 192.012 .077 . \operatorname{rrr}$ & PRIME & PRIME COMPUTER, INC. \\
\hline $\mathrm{C} * 192.012 .078 . \operatorname{rrr}$ & GENNET & GENENTECH NET \\
\hline$C * 192.012 .079 . \operatorname{rrr}$ & SLI & SOFTWARE LEVERAGE INC. \\
\hline R 192.012.080.rrr & CAEN & $\mathrm{UMICH}-\mathrm{CAEN}$ \\
\hline R 192.012.081.rrr & YALE-RING-NET & YALE RESEARCH RING \\
\hline C 192.012.082.rrr & $\mathrm{CU}-\mathrm{CC}-\mathrm{NET}$ & Columbia CC Net \\
\hline G 192.012.083.rrr & UCDLA-EXNET & UCDLA EXPERIMENTAL NET \\
\hline G 192.012.084.rrr & UCDLA-PCNET & UCDLA PERSONAL NET \\
\hline G 192.012.085.rrr & UCDLA-OPNET & UCDLA OPTICAL DISK \\
\hline G $192.012 .086 . \operatorname{rrr}$ & UCDLA-RADNET & UCDLA PACKET RADIO \\
\hline G 192.012.087.rrr & UCDLA-CSLNET & UCDLA STATE LIBRARY \\
\hline$R * 192.012 .088 . \operatorname{rrr}$ & RUTGERS-NWK & RUTGERS, NEWARK \\
\hline
\end{tabular}

[39, MK68]

[TONY]

[39, SL10]

[39, RSM1 ]

[AB20 ]

[AB20 ]

[RG12]

[39, MSM1 ]

[39, MSM1 ]

[39, MSM1 ]

$[39, \mathrm{BJN} 1]$

$[39, \mathrm{JXB}]$

$[39, \mathrm{CYH}]$

[AWS 3 ]

[39, KO11]

[ JL15 ]

$[39, \mathrm{CBD}]$

$[39, \mathrm{CBD}]$

$[39, \mathrm{CBD}]$

[39, CBD ]

$[39, \mathrm{CBD}]$

$[39$, GLH5 $]$

[CAK]

[4, JR15 ]

[39, MJM2 ]

[39, MJM2 ]

[MJM2 ]

$[4, \mathrm{THD}]$

[39, BM4 0 ]

[LRB ]

[LRB ]

[ $\mathrm{RHC} 3$ ]

[RHC3 ]

[39, TM86]

[GAL5]

[39, AKH5 ]

[FS37]

[39, SM96]

[MG58]

[HWB ]

[HML1]

[39, BC14]

[CL 64]

[CL64]

[CL64]

[CL64]

[CL 64 ]

[DB150] 
R 192.012.089.rrr

R 192.012.090.rrr

$192.012 .091 . r r r$

R 192.012.092.rrr $192.012 .093 . r r r$ $192.012 .094 . r r r$ $192.012 .095 . \operatorname{rrr}$ $192.012 .096 . r r r$ $192.012 .097 . r r r$ $192.012 .098 . r r r$ $192.012 .099 . \operatorname{rrr}$ $192.012 .100 . r r r$ $R * 192.012 .101 . r r r$ G 192.012.102.rrr R 192.012.103.rrr R 192.012.104.rrr R 192.012.105.rrr R 192.012.106.rrr R 192.012.107.rrr R 192.012.108.rrr $R \star 192.012 .109$ rrr G 192.012.119.rrr D 192.012.120.rrr $R * 192.012 .121 . r r r$ $R * 192.012 .122 . r r r$ G 192.012.123.rrr D 192.012.124.rrr D 192.012.125.rrr D 192.012.126.rrr R 192.012.127.rrr R 192.012.128.rrr C*192.012.129.rrr R 192.012.130.rrr R 192.012.131.rrr R 192.012.132.rrr R 192.012.133.rrr R 192.012.134.rrr R 192.012.135.rrr R 192.012.136.rrr R 192.012.137.rrr R 192.012.138.rrr D 192.012.139.rrr $R * 192.012 .140 . r r r$ R 192.012.141.rrr $C \star 192.012 .142 . r r r$ $C \star 192.012 .143 . r r r$ R 192.012.144.rrr
SBCS-CSDEPT-1 SB Computer Science SBCS-CSDEPT-2 SB Computer Science RPICSNETO RPICS-LOCALNET-O

RPICSNET1 RPICS-LOCALNET-1

Unassigned Unassigned

Unassigned

Unassigned

Unassigned

Unassigned

Unassigned

Unassigned

Unassigned

OSU-CGRG

AMES-NAS-HY

CSU-USCETHER

CSUNRELETHER

CSU-ASYNC

CSU-LANCE

CSU-ATMOS

CSU-UCC-ETHER

$192.012 .118 . \operatorname{rrr}$ ICST

MITRE-B-NET

FSUCS

FSUCS2

AMES-CCE-NET

ETL-LAN

CRDEC-NET 1

CRDEC-NET2

LL-MI-NET

AITAC-ADMIN

SYM-CAN

SDC-SM

SAC-ADMIN

LLL-MON

LLL-TUE

LLL-WED

LLL-THU

LLL-FRI

LLL-SAT

LLL-SUN

JTELS-BEN-GW

INFERENCE

CSS-ETHER

SENTRY

VSHIC-NET

ECRCNET
Unassigned

Unassigned

Unassigned

Unassigned

Unassigned

Unassigned

Unassigned

OSU Computer Graphics

AMES NAS HY NET

Colorado State Univ Nets

Colorado State Univ Nets

Colorado State Univ Nets

Colorado State Univ Nets

Colorado State Univ Nets

Colorado State Univ Nets

Colorado State Univ Nets

ICST Network

MITRE BEDFORD ETHER

FSU COMPUTER SCIENCE 1

FSU COMPUTER SCIENCE 2

AMES CCF NETWORK

ETL LOCAL AREA NET

CRDEC-NET1

CRDEC-NET2

LL-Machine Intell.

SRI-AITAC ADMIN NET

Symbolics/Canada

SDC Santa Monica

SRI-SAC ADMIN NET

LLL Open Labnet-1

LLL Open Labnet-2

LLL Open Labnet-3

LLL Open Labnet-4

LLL Open Labnet-5

LLL Open Labnet- 6

LLL Open Labnet-7

JUMPS Teleprocessing

INFERENCE

CSS Workstation Net 2

Sentry Adv. Prod. Net

Sentry VSHIC Test

ECRC Internet

RCA-CADNET
[ JS268 ]

[ JS268]

[MS 9]

[MS 9]

[NIC]

[NIC]

[NIC]

[NIC]

[NIC]

[NIC]

[NIC]

[NIC]

[39, KS62 ]

[MF 31]

[RB218]

[RB218]

[RB218]

[RB218]

[RB218]

[RB218]

[RB218]

[39, JCN2 ]

[BSW]

[TB4]

[TB4]

[39, MSM1 ]

[39, WWS ]

[39, JY11]

[39, JY11]

$[39, \mathrm{GAA}]$

[39, $\mathrm{VDC} 1]$

[MMH5 ]

[CAS ]

$[39, \mathrm{KMC} 3]$

[39, BANDY ]

[39, BANDY]

[39, BANDY]

[39, BANDY]

[39, BANDY]

[39, BANDY]

[39, BANDY]

[RR2 6]

[DGT 6]

[RA11]

[LL56 ]

[LL56]

[39, PD39]

[39, RG92 ]

Romano \& Stahl

[Page 20] 


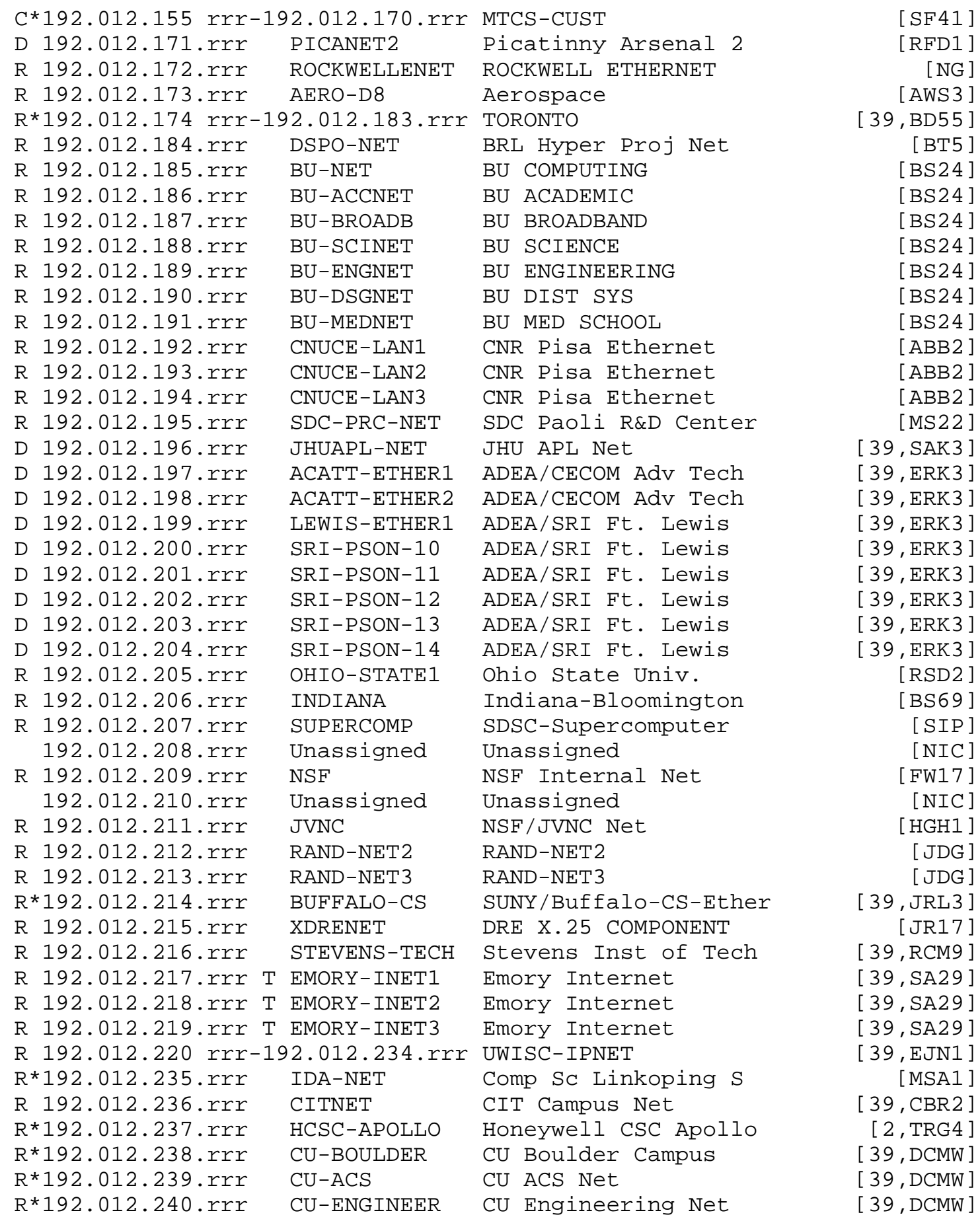




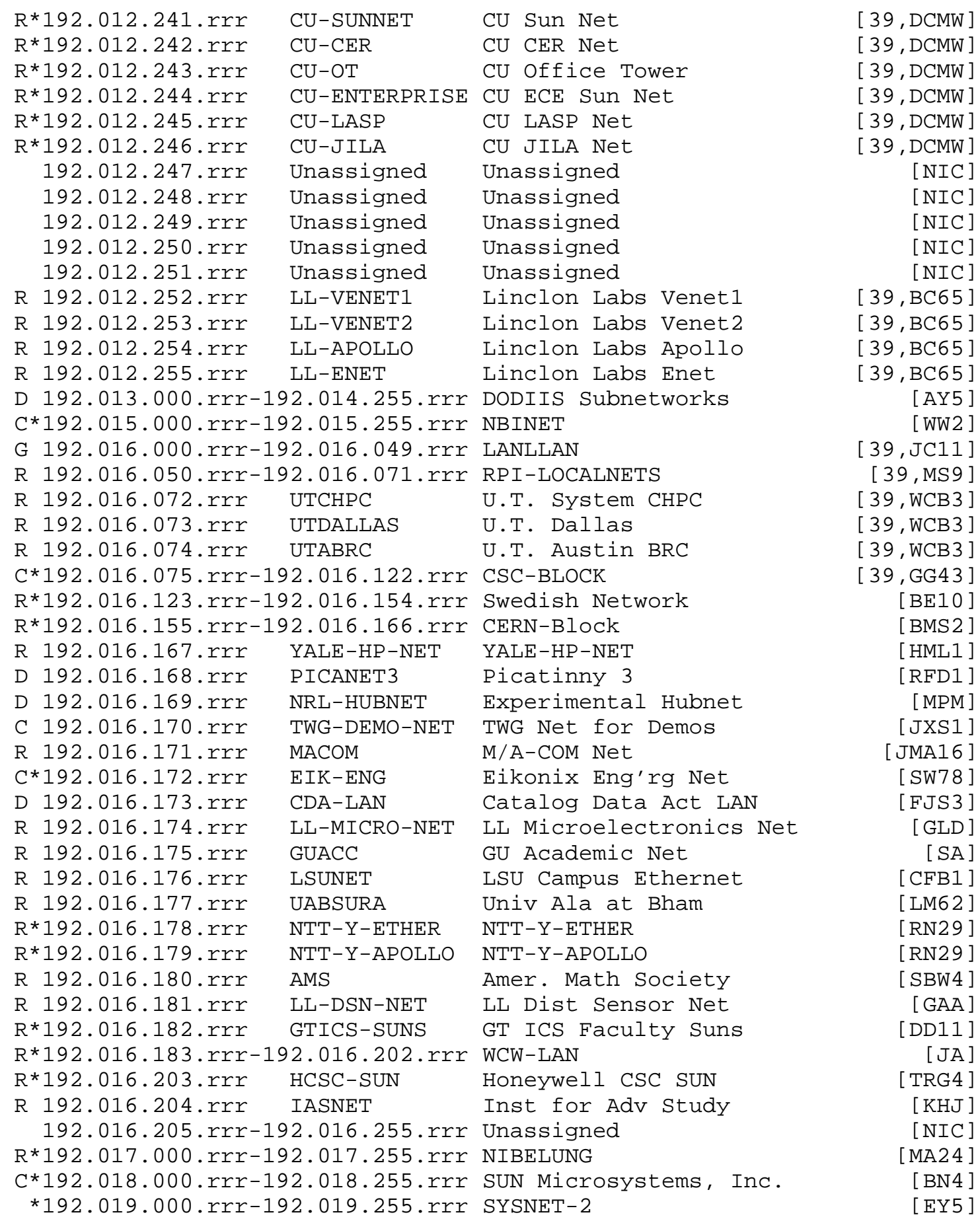




\begin{tabular}{|c|c|c|c|c|}
\hline \multicolumn{4}{|c|}{$\mathrm{C} \star 192.020 .000 . \operatorname{rrr}-192.020 .255 . \operatorname{rrr} \mathrm{ATT}-\mathrm{MD}-\mathrm{NET}$} & {$[39, \mathrm{MH} 82]$} \\
\hline & $* 192.021 .000 . \operatorname{rrr}-$ & $92.021 .255 . \operatorname{rrr}$ & FORMATIVE & {$[\mathrm{SAB} 17]$} \\
\hline & $192.022 .000 . r r r-$ & $2.022 .255 . \operatorname{rrr}$ & APPLICON & [AS90] \\
\hline & $192.023 .000 . r r r-$ & $2.023 .255 . \operatorname{rrr}$ & FACTNET & [ JCB 4 2 ] \\
\hline & $192.024 .000 . r r r-$ & $2.024 .255 . \operatorname{rrr}$ & CHROMATICS & [RB219] \\
\hline & $192.025 .000 . \operatorname{rrr}-$ & $2.025 .255 . \operatorname{rrr}$ & Hewlett Packard & [SI 8 ] \\
\hline & $192.026 .000 . \operatorname{rrr}$ & ACSAD & ACSAD Network & [SLH19] \\
\hline & $192.026 .001 . \operatorname{rrr}$ & $\mathrm{MCC}-\mathrm{DB} 1-\mathrm{NET}$ & MCC DB1 Network & [CBD ] \\
\hline & $192.026 .002 . \operatorname{rrr}$ & $\mathrm{MCC}-\mathrm{DB} 2-\mathrm{NET}$ & MCC DB2 Network & {$[\mathrm{CBD}]$} \\
\hline & $192.026 .003 . \operatorname{rrr}$ & $\mathrm{MCC}-\mathrm{DB} 3-\mathrm{NET}$ & MCC DB3 Network & $\mathrm{BD}]$ \\
\hline & $192.026 .004 . \operatorname{rrr}$ & $\mathrm{MCC}-\mathrm{DB} 4-\mathrm{NET}$ & MCC DB4 Network & $\mathrm{BD}]$ \\
\hline & $192.026 .005 . \operatorname{rrr}$ & $\mathrm{MCC}-\mathrm{DB} 5-\mathrm{NET}$ & MCC DB5 Network & {$[\mathrm{CBD}]$} \\
\hline $\mathrm{R}$ & $192.026 .006 . \operatorname{rrr}$ & $\mathrm{MCC}-\mathrm{DB} 6-\mathrm{NET}$ & MCC DB6 Network & $\mathrm{BD}]$ \\
\hline $\mathrm{R}$ & $192.026 .007 . \operatorname{rrr}$ & SPAWAR & SPARWAR Systems Command & [ JK7 ] \\
\hline & $192.026 .008 . \operatorname{rrr}$ & $\mathrm{SAIC}-\mathrm{CPVB}$ & SAIC-CPVB & [MW4 9] \\
\hline & *192.026.009.rrr & ICOT & ICOT Local Network & [ST13] \\
\hline & $192.026 .010 . \operatorname{rrr}$ & GALLAUDET & GALLAUDET UNIVERSITY & {$[\mathrm{KBC}]$} \\
\hline & $192.026 .011 . \operatorname{rrr}$ & NRL-HUBNET1 & Experimental Hubnet 1 & IPM ] \\
\hline & $192.026 .012 . \operatorname{rrr}$ & NRL-HUBNET2 & Experimental Hubnet 2 & {$[\mathrm{MPM}]$} \\
\hline & $192.026 .013 . \operatorname{rrr}$ & NRL-HUBNET3 & Experimental Hubnet 3 & {$[\mathrm{MPM}]$} \\
\hline & $192.026 .014 . \operatorname{rrr}$ & NRL-HUBNET 4 & Experimental Hubnet 4 & {$[\mathrm{MPM}]$} \\
\hline & $192.026 .015 . \operatorname{rrr}$ & NRL-HUBNET 5 & Experimental Hubnet 5 & {$[\mathrm{MPM}]$} \\
\hline & $192.026 .016 . \operatorname{rrr}$ & NRL-HUBNET 6 & Experimental Hubnet 6 & {$[\mathrm{MPM}]$} \\
\hline & $192.026 .017 . \operatorname{rrr}$ & NRL-HUBNET 7 & Experimental Hubnet 7 & {$[\mathrm{MPM}]$} \\
\hline & $192.026 .018 . \operatorname{rrr}$ & NRL-HUBNET 8 & Experimental Hubnet 8 & {$[\mathrm{MPM}]$} \\
\hline & $192.026 .019 . \operatorname{rrr}$ & NRL-HUBNET 9 & Experimental Hubnet 9 & {$[\mathrm{MPM}]$} \\
\hline & $192.026 .020 . \operatorname{rrr}$ & Unassigned & Unassigned & {$[\mathrm{NIC}]$} \\
\hline & $192.026 .021 . \operatorname{rrr}$ & $S D C-P R C-S W$ & SDC/PAOLI SOFT TECH & [MS 22 ] \\
\hline & $192.026 .022 . \operatorname{rrr}$ & $S D C-P R C-L B S$ & SDC/PAOLI ARTIF INT & [MS22] \\
\hline & $192.026 .023 . \operatorname{rrr}$ & $S D C-P R C-S A$ & SDC/PAOLI SYS ARCH & [MS 22 ] \\
\hline & $192.026 .024 . \operatorname{rrr}$ & $S D C-P R C-C R$ & SDC/PAOLI COMP RES & [MS 22 ] \\
\hline & $192.026 .025 . \operatorname{rrr}$ & LUCID & Lucid Network & [BM68 ] \\
\hline & $192.026 .026 . \operatorname{rrr}$ & NRL-FIBER & NRL Fiber Optic Net & [WF 3 ] \\
\hline & $192.026 .027 . \operatorname{rrr}$ & ROCKEFELLER & ROCKEFELLER UNIV & {$[39, \mathrm{MK} 38]$} \\
\hline & *192.026.028.rrr - & $92.026 .047 . \operatorname{rrr}$ & EPFL & {$[Y X D]$} \\
\hline & *192.026.048.rrr & DART-ETHER & Dartmouth Ethernet & {$[\mathrm{SC} 59]$} \\
\hline & $192.026 .049 . \operatorname{rrr}$ & DUNET & U of Denver Network & {$[39, \mathrm{WE} 12]$} \\
\hline & *192.026.050.rrr- & $92.026 .082 . \operatorname{rrr}$ & Silicon Graphics Inc & [RB221] \\
\hline & $192.026 .083 . \operatorname{rrr}$ & $\mathrm{CSM}-\mathrm{NET}$ & Colorado School of Mines & [KL31] \\
\hline & $192.026 .084 . \operatorname{rrr}$ & NPRDC-FTC & NPRDC-FTC Remote Ethernet & [ LRB ] \\
\hline & $192.026 .085 . \operatorname{rrr}$ & NUSAN & NU Supercomp Access Net & [EEW6] \\
\hline & $192.026 .086 . \operatorname{rrr}$ & PHYSICS-SAC & NU Physics & [EEW6] \\
\hline & $192.026 .087 . \operatorname{rrr}$ & $\mathrm{MS}-\mathrm{SAC}$ & NU Material Science SAC & [EEW6] \\
\hline & $192.026 .088 . \operatorname{rrr}$ & YALE-ENG-NET & YALE-ENG-NET & [ LFO ] \\
\hline & $192.026 .089 . \operatorname{rrr}$ & JTELS-BEN1-GW & JTELS-BEN1-GW & [RR26] \\
\hline & $\star 192.026 .090 . \operatorname{rrr}$ & SYNTELNET-A & Syntelligence IPNET-A & [RAR22 ] \\
\hline & $+192.026 .091 . \operatorname{rrr}$ & $\mathrm{KDD}$ & KDD Research Net & [TA24] \\
\hline & *192.026.092.rrr & WRIGHT & Wright State University & [ JLS4 5] \\
\hline
\end{tabular}




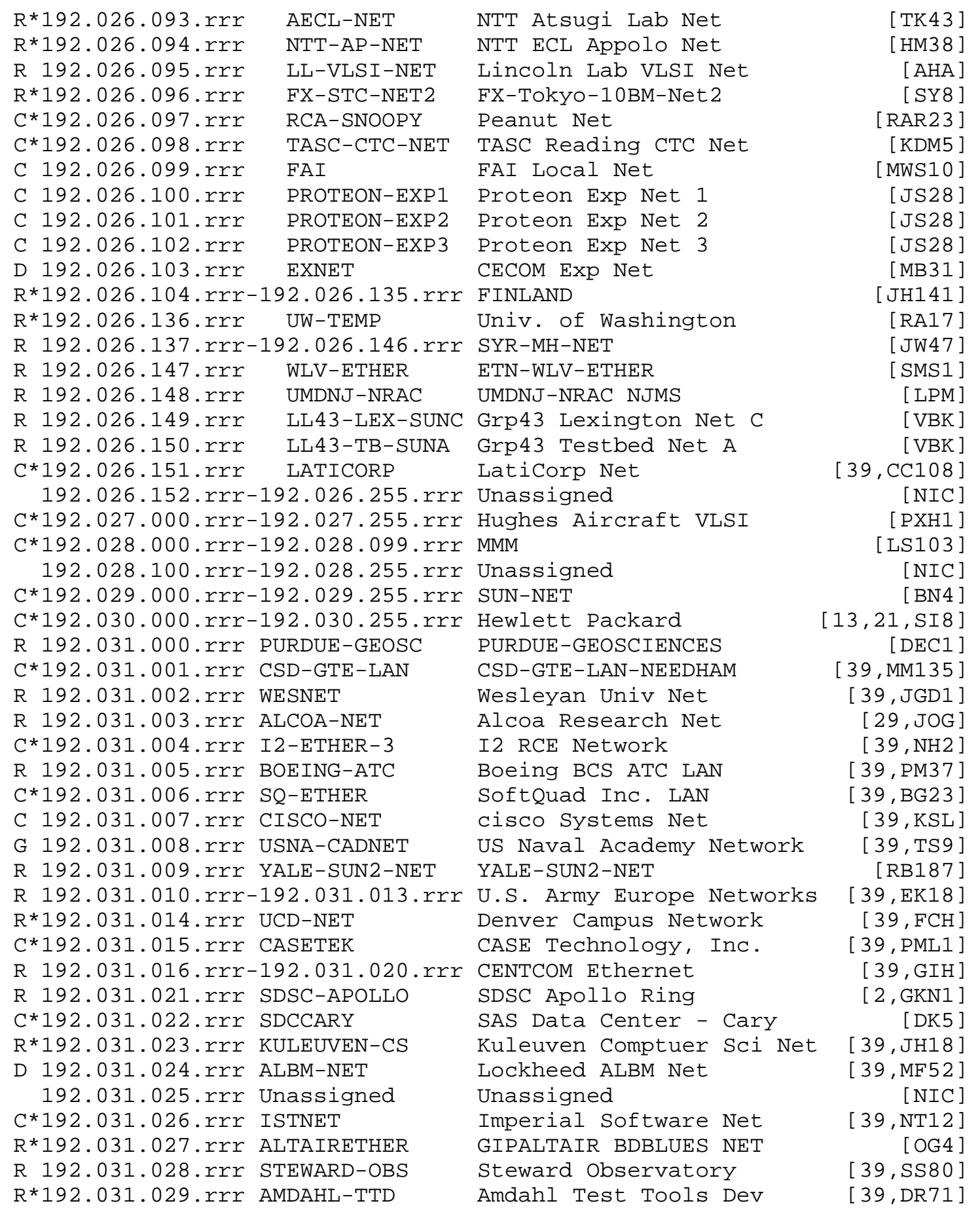




\begin{tabular}{|c|c|c|c|}
\hline R 192.031.030.rrr & $A D S-D C$ & ADS Washington & {$[39, \mathrm{JTN}]$} \\
\hline$C \star 192.031 .031 . \operatorname{rrr}$ & AXION-NET & BT Axion Network & {$[39, \mathrm{NT} 13]$} \\
\hline$C \star 192.031 .032 . \operatorname{rrr}$ & $-192.031 .036 \cdot \operatorname{rrr}$ & NSKK Local Area Network & {$[39, \mathrm{AK} 36]$} \\
\hline$C \star 192.031 .037 . \operatorname{rrr}$ & SDAPOLL & SAS Data Center - Cary & [DK5 ] \\
\hline$C \star 192.031 .038 . \operatorname{rrr}$ & TIATSP INE & TI Attleboro Spine & [WDR7 ] \\
\hline$R * 192.031 .039 . \operatorname{rrr}$ & BAYLOR & Baylor Univ Network & {$[39, \mathrm{BL} 31]$} \\
\hline R 192.031.040.rrr & YALE-SUN3-NET & YALE-SUN3-NET & [RB187] \\
\hline R 192.031.041.rrr & YALE-RT-NET & YALE-RT-NET & {$[\mathrm{RB} 187]$} \\
\hline R 192.031.042.rrr & YALE-RT2-NET & YALE-RT2-NET & [RB187] \\
\hline $192.031 .043 . \operatorname{rrr}$ & CNSNET & Caltech - CNS Biology Net & {$[39, \mathrm{DC} 99]$} \\
\hline C $192.031 .044 . \operatorname{rrr}$ & $\mathrm{MRC}-\mathrm{NET}$ & McLean Research Center & [WLG7 ] \\
\hline R 192.031.045.rrr & WILLIAMS & Williams College & {$[39$, RW101] } \\
\hline $192.031 .046 . \operatorname{rrr}$ & Unassigned & Unassigned & {$[\mathrm{NIC}]$} \\
\hline R 192.031.047.rrr & $-192.031 .061 \cdot \operatorname{rrr}$ & Bay Area Regional Network & {$[\mathrm{AB} 71]$} \\
\hline$R * 192.031 .062 . \operatorname{rrr}$ & SRI-CAM & SRI Cambridge UK & {$[39$, AGS 5$]$} \\
\hline R 192.031.063.rrr & SCUBED-BBONE & SCUBED-BBONE-NET & {$[39, \mathrm{TH} 60]$} \\
\hline R 192.031.064.rrr & S3-RESEARCH & SCUBED-RESEARCH-NET & {$[39, \mathrm{TH} 60]$} \\
\hline R 192.031.065.rrr & S3-FIBER-NET & SCUBED-FIBER-NET & {$[39, \mathrm{TH} 60]$} \\
\hline R 192.031.066.rrr & S3-ABQNET & SCUBED-ABQNET & {$[39, \mathrm{TH} 60]$} \\
\hline R 192.031.067.rrr & S3-SLIP-NET & SCUBED-SLIP-NET & {$[39, \mathrm{TH} 60]$} \\
\hline R 192.031.068.rrr & S3-THIN-NET & SCUBED-THIN-NET & {$[39, \mathrm{TH} 60]$} \\
\hline R 192.031.069.rrr & S3-BBONE2-NET & SCUBED-BBONE2-NET & {$[39, \mathrm{TH} 60]$} \\
\hline R 192.031.070.rrr & S3-ETHER2-NET & SCUBED-ETHER2-NET & {$[39, \mathrm{TH} 60]$} \\
\hline R 192.031.071.rrr & S3-ETHER3-NET & SCUBED-ETHER3-NET & {$[39, \mathrm{TH} 60]$} \\
\hline R 192.031.072.rrr & S3-ETHER4-NET & SCUBED-ETHER4-NET & {$[39, \mathrm{TH} 60]$} \\
\hline$C \star 192.031 .073 . \operatorname{rrr}$ & MTEL-APOLLO & M/A-COM MTEL Apollo Net & {$[39, \mathrm{JF} 77]$} \\
\hline $.031 .074 . \operatorname{rrr}$ & GSSD-APOLLO & M/A-COM GSSD Apollo Net & {$[39, \mathrm{PC} 55]$} \\
\hline D $192.031 .075 . \operatorname{rrr}$ & $\mathrm{HQDA}-\mathrm{AI}$ & Pentagon Army AI Net & {$[39, \mathrm{DH} 23]$} \\
\hline D $192.031 .076 . \operatorname{rrr}$ & CSTLNET & Combat Sys Tech Lab & {$[\mathrm{MP} 20]$} \\
\hline$C * 192.031 .077 . \operatorname{rrr}$ & MAPNET & Mervine \& Pallesen Net & {$[23, \mathrm{BH} 80]$} \\
\hline$C * 192.031 .078 . \operatorname{rrr}$ & WELLSNET-A1 & Wells Fargo IPNET-A1 & {$[39, \mathrm{JN} 47]$} \\
\hline$C \star 192.031 .079 . \operatorname{rrr}$ & WACHOVIANET-A1 & Wachovia IPNET-A1 & {$[39, \mathrm{PMH} 3]$} \\
\hline R 192.031.080.rrr & KSUNET & KSU Campus Network & {$[\mathrm{BAV}]$} \\
\hline R 192.031.081.rrr & HUSKERNET & UNL Campus Network & [MM1 4 7] \\
\hline D $192.031 .082 . \operatorname{rrr}$ & HQEIS & HQ AFSC EIS & {$[39, \mathrm{SMK} 2]$} \\
\hline $.031 .083 . \operatorname{rrr}$ & OSUNET & OSU Campus Network & [PW37] \\
\hline$C \star 192.031 .084 . \operatorname{rrr}$ & CUBI & Cubicomp Corporation Net & {$[2, \mathrm{SFJ}]$} \\
\hline C $192.031 .085 . \operatorname{rrr}$ & CLINET & Computational Logic Net & {$[39$, WAH11] } \\
\hline R 192.031.086.rrr & RAZORNET & UAF Campus Network & [DLM3 4] \\
\hline R 192.031.087.rrr & HARC-NET & Houston Area Rsch Ctr Net & [DN22 ] \\
\hline R 192.031.088.rrr & BCMTECH-NET & BCM Technologies Network & {$[39, \mathrm{SB} 98]$} \\
\hline R 192.031.089.rrr & MIAMI & University of Miami & {$[39, \operatorname{HWP} 2]$} \\
\hline$R * 192.031 .090 . \operatorname{rrr}$ & MORAVIAN & Moravian College & [ JPS17] \\
\hline $192.031 .091 . \operatorname{rrr}$ & Unassigned & Unassigned & {$[\mathrm{NIC}]$} \\
\hline R 192.031.092.rrr & CIT-CONTROL & Caltech Control Lab & {$[39, \mathrm{JD} 27]$} \\
\hline R 192.031.093.rrr & CIT-SRLNET & Caltech SRL Network & {$[39, \mathrm{CJL} 2]$} \\
\hline R 192.031.094.rrr & KEHNET & Comp Science and Eng Ethe & net $[\mathrm{PLH} 8]$ \\
\hline$R * 192.031 .095 . \operatorname{rrr}$ & UCCNET & UC Corporate, Admin Net & {$[39, \mathrm{AC} 42]$} \\
\hline
\end{tabular}


G 192.031.096.rrr ORNL-OSTINET

R 192.031.097.rrr KSU-NET

D 192.031.098.rrr PBAS-BEN2-GW

R 192.031.099.rrr ISUNET

D 192.031.100.rrr GUNTER-LAN

$\mathrm{R} \star 192.031 .101 . \operatorname{rrr} \mathrm{TSU}-\mathrm{NET}$

$\mathrm{R}$ 192.031.102.rrr M2C-NET

R 192.031.103.rrr P-TO-P-NET

R 192.031.104.rrr PSCSURA

$R * 192.031 .105 . r r r$ UCC-PRO-UCB

D 192.031.106.rrr NSWSES-NAVY

R 192.031.107.rrr JAYHAWKNET

$\mathrm{R} * 192.031 .108 . r r r$ UCFCSNET

$\mathrm{R} * 192.031 .109 . r r r$ FREDONIA

C*192.031.110.rrr ADCAPOLL

$R * 192.031 .111 . r r r$ AIRMICS

$R * 192.031 .112 . r r r$ TRINCOLL

C 192.031.113.rrr MONET

$C \star 192.031 .114 . r r r$ DRINET

C*192.031.115.rrr FIRENET-AI

$R \star 192.031 .116 . r r r-192.031 .124 . r r r$

$R \star 192.031 .125 . r r r-192.031 .144 . r r r$

R 192.031.145.rrr SIGNET

R 192.031.146.rrr UCR

D 192.031.147.rrr NUWESNET

C*192.031.148.rrr AIGNET-AI

$\mathrm{C} * 192.031 .149 . r r r$ WACNET-AI

$C \star 192.031 .150 . r r r$ STPNET-AI

C*192.031.151.rrr COGNITIONNET

C 192.031.152.rrr ROSENET

R 192.031.153.rrr SALKNET

R 192.031.154.rrr UNMHC-DEV

R 192.031.155.rrr GEOLOGY-NWU

$R * 192.031 .156 . r r r$ CANISIUS-CS

R 192.031.157.rrr RTNET

D 192.031.158.rrr DAITC

R 192.031.159.rrr NYTGCYLAB

D 192.031.160.rrr NUWES-C-NET

R 192.031.161.rrr UCB-UCSC-NET

G $192.031 .162 . \operatorname{rrr}$ DOL-NET

$192.031 .163-223.255 .254 . r r r$

$223.255 .255 . r r r$
OSTI Local Area Network

Kansas State University

PBAS-BEN2-GATEWAY

ISU Campus Network

GUNTER-LAN

Texas Southern Univ Net

Mass Microelectronics Ct Net

CSNET Point to Point Network

PSCSURA

UC Corporate, Admin Net

PORT-HUENEME-CBC

KU Campus Networks

UCF CS Dept. Network

SUC-FREDONIA

Austin Data Ctr APOLLO RING [RC113]

AIRMICS Research Net

Trinity-Hartford

Univ-of-Mo-Net

DRI Engineering Net

Fireman's Fund IPNET-AI

University of Tokyo Net

DUT Network

Small IP Gateway Net

UC Riverside

NUWES-KEYPORT-LAN

AIG IPNET-AI

1st Wachovia IPNET-AI

St. Paul IPNET-AI

CI-Headquarters

Rosetta Network

Salk Institute Net

$\mathrm{U}$ of NM Hypercube Dvlp Net

Northwestern Geology

[DFH2 ]

$[38, \mathrm{MA} 54]$

[BEC5]

$[21, \mathrm{~KB} 60$ ]

$[39, \mathrm{CO} 16]$

$[39$, JM2 92$]$

[39, FD18]

$[39, \mathrm{PGM}]$

[39, WS 73]

[RM125]

[39, RK51]

[39, РMH3 ]

$[39, \operatorname{RLP} 30]$

$[39$, DW93 ]

$[39, \mathrm{SC} 54]$

$[39, \mathrm{JOO}]$

$[39, \mathrm{KDZ}]$

$[39$, EEW6 ]

Canisius Comp Science Net [39,MS101]

C3P Ether Cube

$[39, \mathrm{SC} 81]$

Defense Appl Info Tech Ctr [39, CG24]

NYTGCYLAB

NUWES-KEYPORT-LAN

UCB-UCSC 56K Backup

Department of Labor Net

Unassigned

Reserved
$[39, \operatorname{SS} 110]$

[RM125 ]

[CF 4$]$

$[39, \mathrm{DD} 47]$

[NIC]

[ JBP ] 
Other Reserved Internet Addresses

* Internet Address Name Network

- ---------------- ---- ------$224.000 .000 .000-239.255 .255 .255$ Multicast $240.000 .000 .000-255.255 .255 .255$ Reserved
References

$---------$

$[11, \mathrm{JBP}]$

[ JBP ] 


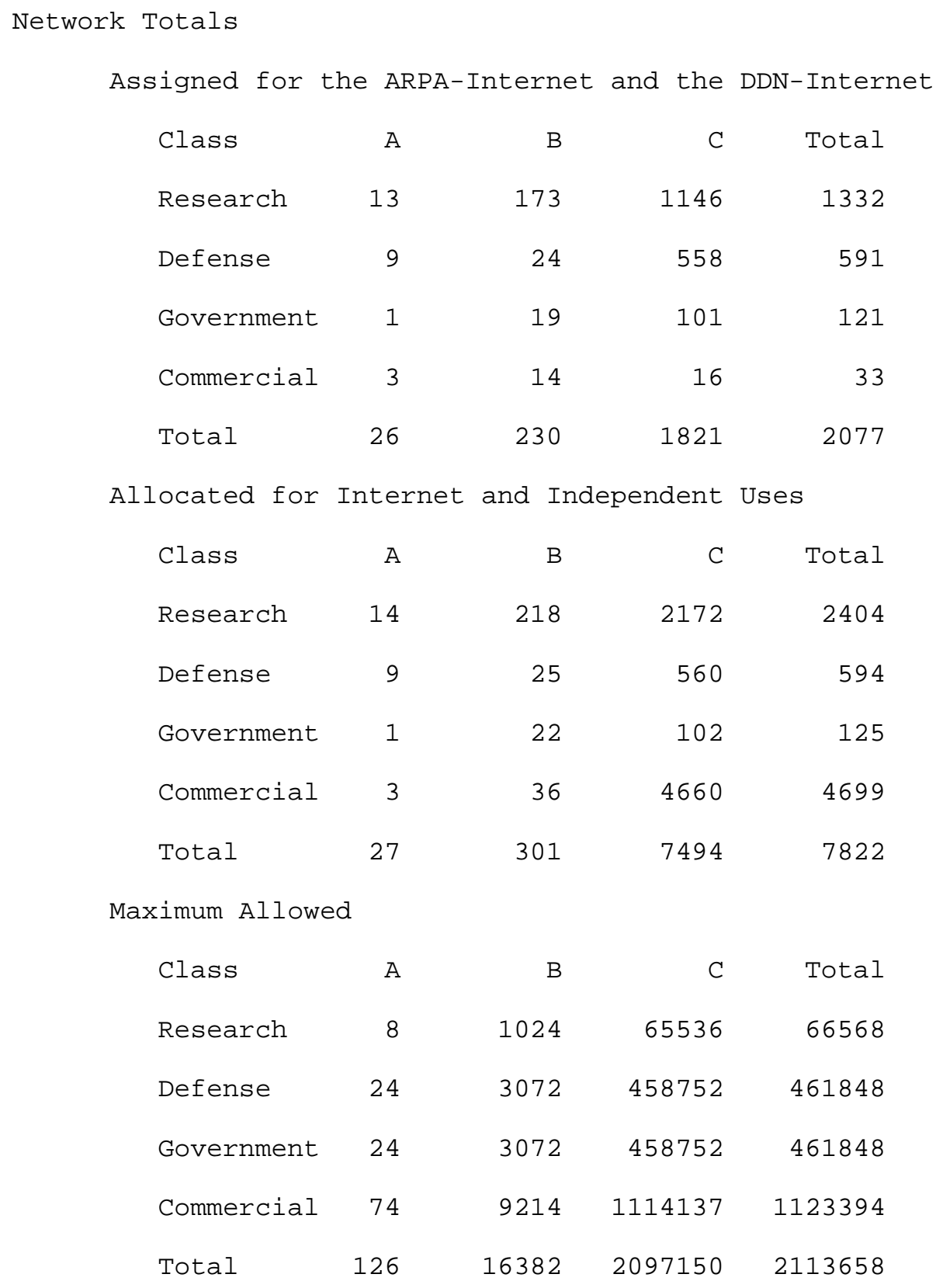




\section{AUTONOMOUS SYSTEM NUMBERS}

The Exterior Gateway Protocol (EGP) [33,35] specifies that groups of gateways may form autonomous systems. The EGP provides a 16-bit field for identifying such systems. The values of this field are registered here.

Autonomous system Numbers:

\begin{tabular}{|c|c|}
\hline Decimal & Name \\
\hline 0 & Reserved \\
\hline 1 & The BBN Core Gateways \\
\hline 2 & $\mathrm{DCN}-\mathrm{AS}$ \\
\hline 3 & The MIT Gateways \\
\hline 4 & ISI-AS \\
\hline 5 & Symbolics \\
\hline 6 & HIS-Multics \\
\hline 7 & $\mathrm{UK}-\mathrm{MOD}$ \\
\hline 8 & $\mathrm{RICE}-\mathrm{AS}$ \\
\hline 9 & CMU-ROUTER \\
\hline 10 & CSNET-PDN-AS \\
\hline 11 & HARVARD \\
\hline 12 & NYU-DOMAIN \\
\hline 13 & $\mathrm{BRL}-\mathrm{AS}$ \\
\hline 14 & COLUMBIA-GW \\
\hline 15 & NET DYNAMICS EXP \\
\hline 16 & LBL \\
\hline 17 & PURDUE-CS \\
\hline 18 & UTEXAS \\
\hline 19 & CSS-DOMAIN \\
\hline 20 & $\mathrm{UR}$ \\
\hline 21 & RAND \\
\hline 22 & NOSC \\
\hline 23 & RIACS-AS \\
\hline 24 & AMES-NAS-GW \\
\hline 25 & $\mathrm{UCB}$ \\
\hline 26 & CORNELL \\
\hline 27 & UMDNET \\
\hline 28 & DFVLR-SYS \\
\hline 29 & YALE-AS \\
\hline 30 & SRI-AICNET \\
\hline 31 & $\mathrm{CIT}-\mathrm{CS}$ \\
\hline 32 & STANFORD \\
\hline 33 & $\mathrm{DEC}-\mathrm{WRL}-\mathrm{A} S$ \\
\hline 34 & UDEL-EECIS \\
\hline 35 & MICATON \\
\hline
\end{tabular}

\begin{tabular}{|c|}
\hline \multirow[t]{2}{*}{ References } \\
\hline \\
\hline [ JBP ] \\
\hline [MB ] \\
\hline [DLM1 ] \\
\hline [ LM8 ] \\
\hline [ JKR1 ] \\
\hline [ $\mathrm{CH} 2]$ \\
\hline [ JLM2 3 ] \\
\hline [ RNM1 ] \\
\hline [PGM] \\
\hline [MA ] \\
\hline [RDR4] \\
\hline [SB28] \\
\hline [EF5] \\
\hline [RBN1] \\
\hline [BC14] \\
\hline [ ZSU ] \\
\hline [WG ] \\
\hline [DT50] \\
\hline [ JBC2 ] \\
\hline [RR2 ] \\
\hline [ LB16] \\
\hline [ JDG ] \\
\hline [RLB3 ] \\
\hline [DG28 ] \\
\hline [MF 31] \\
\hline [MK1 7 ] \\
\hline [BN9] \\
\hline [MP 12] \\
\hline [GB 7 ] \\
\hline [ JG 46 ] \\
\hline [PM4 ] \\
\hline [AD22 ] \\
\hline [PA5] \\
\hline [RKJ2] \\
\hline [NMM] \\
\hline [WDL ] \\
\hline
\end{tabular}




\begin{tabular}{|c|c|}
\hline 36 & EGP-TESTOR \\
\hline 37 & NSWC \\
\hline 38 & UIUC \\
\hline 39 & NRL-ITD \\
\hline 40 & MIT-TEST \\
\hline 41 & AMES \\
\hline 42 & THINK-AS \\
\hline 43 & $\mathrm{BNL}-\mathrm{AS}$ \\
\hline 44 & S1-DOMAIN \\
\hline 45 & LLL-TIS-AS \\
\hline 46 & RUTGERS \\
\hline 47 & USC-OBERON \\
\hline 48 & $\mathrm{NRL}-\mathrm{AS}$ \\
\hline 49 & ICST-AS \\
\hline 50 & ORNL-MSRNET \\
\hline 51 & USAREUR-EM-AS \\
\hline 52 & UCLA \\
\hline 53 & NORTHROP -AS \\
\hline 54 & $\mathrm{COA}-\mathrm{FIN}-\mathrm{NET}$ \\
\hline 55 & UPENN-CIS \\
\hline 56 & OPTIMIS-P \\
\hline 57 & $\mathrm{UMN}-\mathrm{REI}-\mathrm{UC}$ \\
\hline 58 & $\mathrm{DREA}-\mathrm{AS}$ \\
\hline 59 & WISC-MADISON-AS \\
\hline 60 & DARPA-BFLY \\
\hline 61 & DEC-MARLBORO-AS \\
\hline 62 & TEKVAXC \\
\hline 63 & $\mathrm{LL}-\mathrm{MI}$ \\
\hline 64 & MITRE-B-AS \\
\hline 65 & LOGNET-AS \\
\hline 66 & ETL-AI \\
\hline 67 & $S D C-P R C-A S$ \\
\hline 68 & LANL-INET-AS \\
\hline 69 & WHARTON-AS \\
\hline 70 & $\mathrm{NLM}-\mathrm{GW}$ \\
\hline 71 & HP-INTERNET-AS \\
\hline 72 & SPAR-AS \\
\hline 73 & WASHINGTON-AS \\
\hline 74 & XDRENET-AS \\
\hline 75 & ANL-AS \\
\hline 76 & $S D C-C A M-A S$ \\
\hline 77 & JHUAP L-AS \\
\hline 78 & $\mathrm{SSDF}-\mathrm{CDC}-\mathrm{GW}$ \\
\hline 79 & $\mathrm{DSPO}-\mathrm{HC}-\mathrm{AS}$ \\
\hline 80 & $\mathrm{GE}-\mathrm{CRD}$ \\
\hline 81 & TUCC-MCNC \\
\hline 82 & TWG-DEMO-AS \\
\hline 83 & PICANET-AS \\
\hline
\end{tabular}

[BP 17]

[MXP 1]

[AKC]

[AP ]

[NC3 ]

[MSM1]

[BJN1]

[GC]

[LWR]

[NAL ]

[RM8 ]

[DRS 4]

[WF 3]

[ JCN2]

[THD ]

[WXD ]

[BXL ]

[RSM1]

[RR2 6]

[ IW5]

[ JXL ]

[HWB]

[GLH5]

[EJN1]

[MB ]

[WM3 ]

[TE2]

[RTL]

[BSW]

[ JR15]

[MMM3 ]

[MXS2 ]

[ JC11]

[GBR]

[JA1]

[RM142]

[RXB]

[RA17]

[JR1 7]

[LW2 6]

[DSR]

[SAK3 ]

[RE22]

[BT5]

[ JC106]

[ JXR]

[ JXS1]

[RFD1] 


\begin{tabular}{|c|c|}
\hline 84 & DTNSRDC-AS1 \\
\hline 85 & AERO-NET \\
\hline 86 & SURANET-AS \\
\hline 87 & INDIANA-AS \\
\hline 88 & PRINCETON-AS \\
\hline 89 & NUSC-CSTLNET-AS \\
\hline 90 & SUN-AS \\
\hline 91 & $\mathrm{RPI}-\mathrm{AS}$ \\
\hline 92 & CLARKSON-AS \\
\hline 93 & FORD-AS \\
\hline 94 & BELVOIR-NET \\
\hline 95 & NUSCLSB1 \\
\hline 96 & JTELS-BEN1-AS \\
\hline 97 & JVNC-AS \\
\hline 98 & ROCKEFELLER-AS \\
\hline 99 & INTEL-IWARP \\
\hline 100 & FMC-CEL \\
\hline 101 & WASH-NSF-AS \\
\hline 102 & $\mathrm{NSF}-\mathrm{HQ}-\mathrm{AS}$ \\
\hline 103 & NWU-A. \\
\hline 104 & COLORADO-AS \\
\hline 105 & GSWD-VMS-AS \\
\hline 106 & ETN-WLV-AS \\
\hline 107 & ECSNET-AS \\
\hline 108 & XEROX-AS \\
\hline 109 & CISCOSYSTEMS \\
\hline 110 & $\mathrm{CCA}-\mathrm{A} \mathrm{S}$ \\
\hline 111 & BOSTONU-AS \\
\hline 112 & CMU-SEI-AS \\
\hline 113 & SCCNET-AS \\
\hline 114 & SESQUINET-AS \\
\hline 115 & $\mathrm{PBAS}-\mathrm{BEN} 2-\mathrm{GW}-\mathrm{AS}$ \\
\hline 116 & BELLCORE-AS \\
\hline 117 & ALBM-NET-AS \\
\hline 118 & NSWSES-NAVY-AS \\
\hline 119 & $\mathrm{AMS}-\mathrm{AS}$ \\
\hline $\begin{array}{c}120-655 \\
65535\end{array}$ & 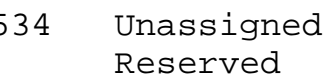 \\
\hline
\end{tabular}

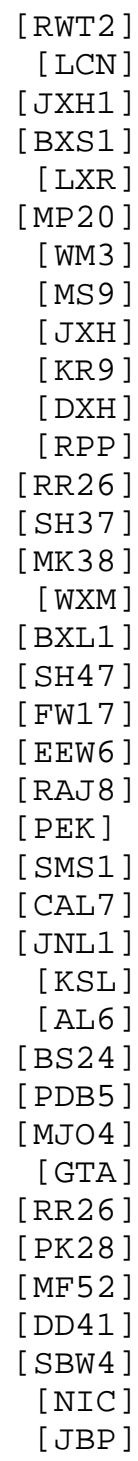




\section{DOCUMENTS}

[1] Aerospace, Internal Report, ATM-83(3920-01)-3, 1982.

[2] Apollo Computer, Inc., "Managing TCP/IP-Based Communication Products", Order No. 008543, Chelmsford, MA, 01824, March 1986.

[3] BBN Proposal No. P83-COM-40, "Packet Switched Overlay to Tactical Multichannel/Satellite systems".

[4] BBN, "Specifications for the Interconnection of a Host and an IMP", Report 1822, Bolt Beranek and Newman, Cambridge, Massachusetts, revised, December 1981.

[5] Chon, K., et al., "SDN: A Computer Network for Korean Research Community", Proc. of the Pacific Computer Communications Symposium, October 1985, pp. 567-570, Seoul, Korea.

[6] Chon, K., et al., "System Development Network", Proc. of TENCON, April 1984, pp. 133-135, Singapore.

[7] Clark, D., "Revision of DSP Specification", Local Network Note 9, Laboratory for Computer Science, MIT, June 1977.

[8] Cohen, D., "On Holy Wars and a Plea for Peace", IEEE Computer Magazine, October 1981.

[9] Comer, D., and T. Narten, "The Cypress Multifunction Packet Switch", Technical Report CSD-TR-575, Computer Science Dept., Purdue University, West LaFayette, IN.

[10] Croft, W. J., "Unix Networking at Purdue", USENIX Conference, 1980 .

[11] Deering, S. E., "Host Extensions for IP Multicasting", RFC-988, Stanford University, December 1985.

[12] Feinler, E., editor, "DDN Protocol Handbook", Network Information Center, SRI International, December 1985.

[13] Feinler, E., editor, "Internet Protocol Transition Workbook", Network Information Center, SRI International, March 1982.

[14] Feinler, E. and J. Postel, eds., "ARPANET Protocol Handbook", NIC 7104, for the Defense Communications Agency by SRI

International, Menlo Park, California, Revised January 1978. 
[15] Harris Corporation, "Harris Ethernet Data Link Reference Manual", Publication No. 0868010-002, Harris Corporation, Computer Systems Divsion, 2101 West Cypress Creek Road, Ft. Lauderdale, FL 33309-1892.

[16] Harris Corporation, "Harris TCP/IP Manager's Guide", Publication No. 0868011-100, Harris Corporation, Computer Systems Divsion, 2101 West Cypress Creek Road, Ft. Lauderdale, FL 33309-1892.

[17] Honeywell CISL, Internal Document, "AFSDSC Hyperchannel RPQ Project Plan".

[18] Honeywell CISL, Internal Document, "Multics MR11 PFS".

[19] Hwang, K., W. J. Croft and G. H. Goble, "A Unix-Based Local Computer Network with Load Balancing", IEEE Computer, April 1982 .

[20] IBM Corporation, "Technical Reference Manual for the IBM PC Network", 6322505, IBM, Boca Raton, Florida, 1984.

[21] IEEE Project 802 Local Area Network Standard, "IEEE Standard 802.3 CSMA/CD Access Method and Physical Layer Specifications", Approved IEEE 802.3-1985 ISO/DIS 8802/3, July 1983.

[22] Korb, J. T., "A Standard for the Transmission of IP Datagrams Over Public Data Networks", RFC-877, Purdue University, September 1983 .

[23] Leach, et al., "The Architecture of an Integrated Local Network", IEEE Journal on Selected Areas in Communications, Vol SAC-1, No. 5, November 1983.

[24] Leffler, Samuel J., et al., "4.2 BSD Network Implementation Notes", July, 1983, University of California, Berkeley.

[25] Macgregor, W., and D. Tappan, "The CRONUS Virtual Local Network", RFC-824, Bolt Beranek and Newman, August 1982.

[26] Mills, D., "Network Time Protocol", RFC-958, M/A-COM Linkabit, September 1985 .

[27] Postel, J., ed., "Internet Protocol - DARPA Internet Program Protocol specification", RFC-791, Information Sciences Institute, September 1981 . 
[28] Prime, "Medusa, The Prime Ethernet", PRIME/WS/AI/86/2, July 1986, Framingham, MA.

[29] Proteon, "Linkway Software: Operating System, Release 7.0", SPD 040-013 and "Linkway Software: IP Packet Forwarder", SPD 040-016. Proteon, Inc., 4 Tech Circle, Natick, MA 01760.

[30] Proteon, "P4200 Gateway User's Guide", 42-040-012. Proteon, Inc., 4 Tech Circle, Natick, MA 01760.

[31] Reed, D., "Protocols for the LCS Network", Local Network Note 3, Laboratory for Computer Science, MIT, November 1976.

[32] Reynolds, J. and J. Postel, "Official Internet Protocols", RFC-1011, Information Sciences Institute, May 1987.

[33] Rosen, E., "Exterior Gateway Protocol" RFC-827, Bolt Beranek and Newman, October 1982 .

[34] Saltzer, J. H., "Design of a Ten-megabit/sec Token Ring Network", MIT Laboratory for Computer Science Technical Report.

[35] Seamonson, L. J., and E. C. Rosen, "STUB" Exterior Gateway Protocol", RFC-888, BBN Communications Corporation, January 1984 .

[36] Shuttleworth, B., "A Documentary of MFENet, a National Computer Network", UCRL-52317, Lawrence Livermore Labs, Livermore, California, June 1977.

[37] Skelton, A., S. Holmgren, and D. Wood, "The MITRE Cablenet Project", IEN-96, April 1979.

[38] Sun Microsystems, "Networking on the Sun Workstation", Part No: 800-1324-03, Revision B of 17 February 1986. Sun Microsystems, Inc., 2550 Garcia Avenue, Mountain View, CA 94043.

[39] "The Ethernet, A Local Area Network: Data Link Layer and Physical Layer Specification", AA-K759B-TK, Digital Equipment Corporation, Maynard, MA. Also as: "The Ethernet - A Local Area Network", Version 1.0, Digital Equipment Corporation, Intel Corporation, Xerox Corporation, September 1980. And: "The Ethernet, A Local Area Network: Data Link Layer and Physical Layer Specifications", Digital, Intel and Xerox, November 1982. And: XEROX, "The Ethernet, A Local Area Network: Data Link Layer and Physical Layer Specification", 
X3т51/80-50, Xerox Corporation, Stamford, Ст., October 1980.

[40] The High Level Protocol Group, "A Network Independent File Transfer Protocol", INWG Protocol Note 86, December 1977.

[41] Whelan, D., "The Caltech Computer Science Department Network", 5052:D F:82, Caltech Computer Science Department, 1892.

[42] XEROX, "Internet Transport Protocols", XSIS 028112, Xerox Corporation, Stamford, Connecticut, December 1981. 


\section{CONTACTS}

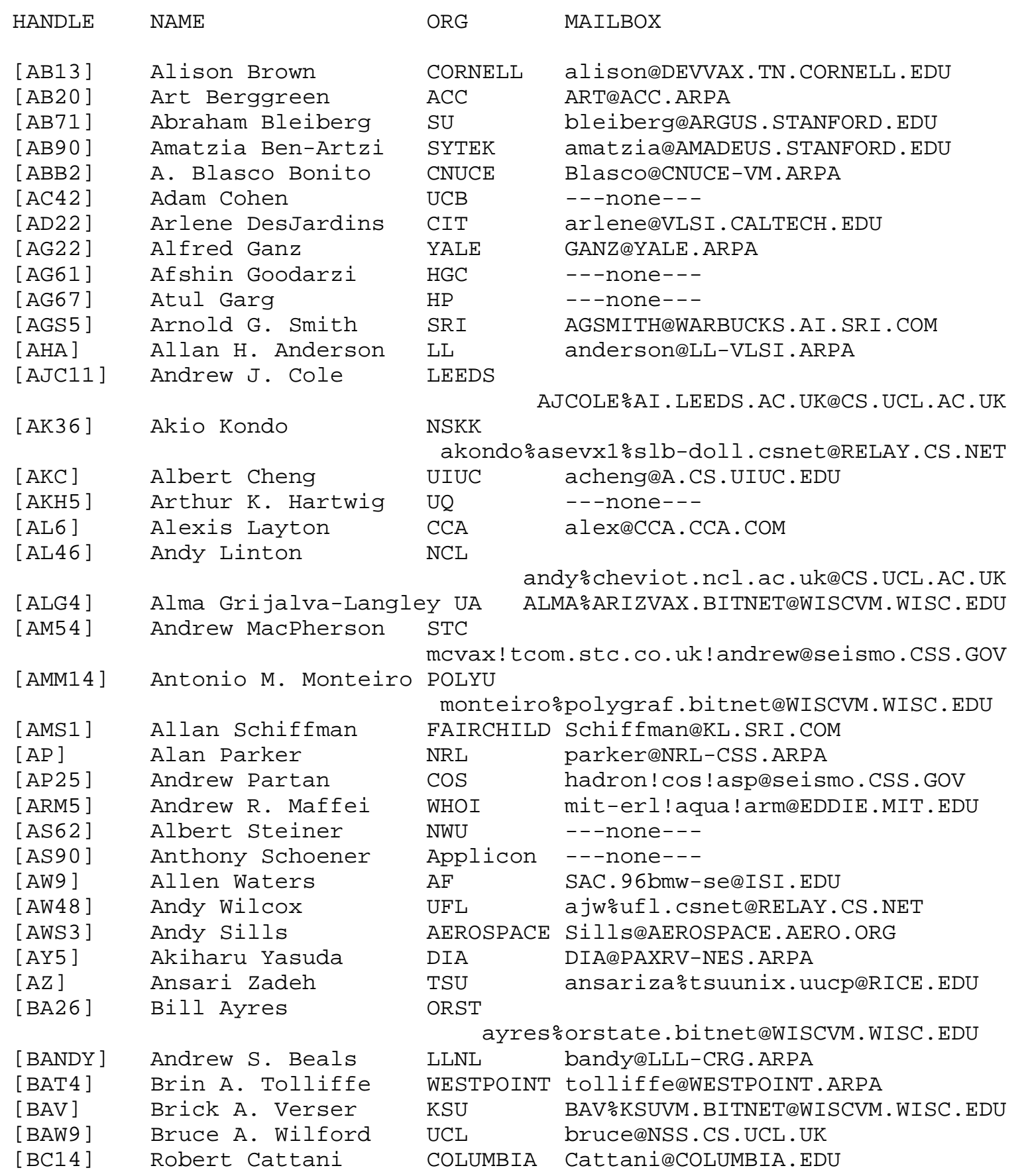




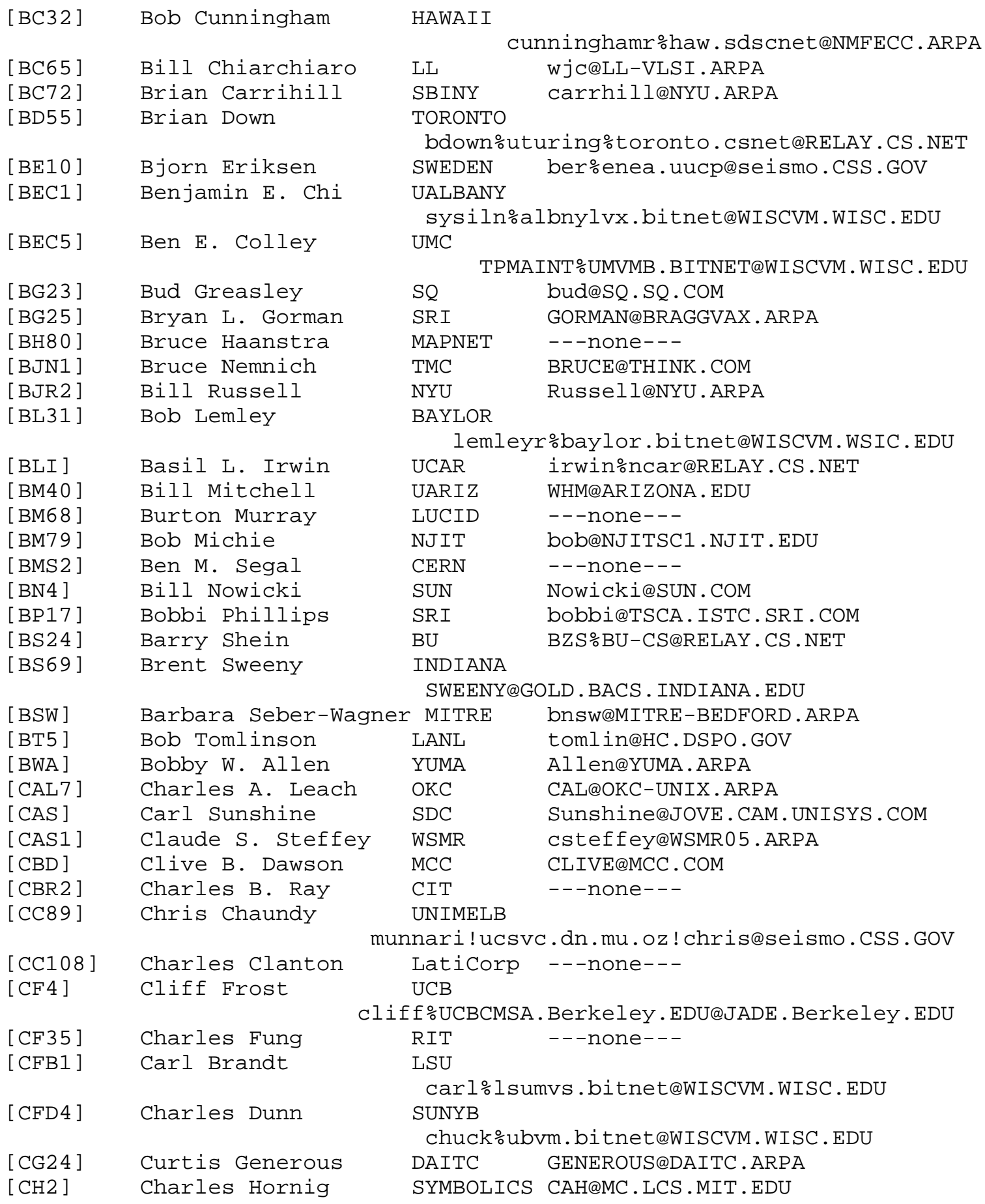




\begin{tabular}{|c|c|c|c|}
\hline [CJ38 ] & Chris Johnson & \multicolumn{2}{|c|}{$\mathrm{NU}$} \\
\hline [CJL2 ] & Carl J. Lydick & CIT & Carl@CITHEX.CALTECH.EDU \\
\hline [CJW2 ] & Cliff Weinstein & LL & cjw@LL-SST.ARPA \\
\hline [CL64] & Clifford A. Lynch & \multicolumn{2}{|c|}{$\begin{array}{l}\text { BERKELEY } \\
\text { ucdla\%ucbtopaz.cc@UCBARPA.Berkeley. EDU }\end{array}$} \\
\hline [CLH3 ] & Charles Hedrick & RUTGERS & Hedrick@RED.RUTGERS.EDU \\
\hline [CMC 6 ] & Chai M. Chow & WPAFB & chowcm@WPAFB-AMS1 . ARPA \\
\hline$[\mathrm{CMR}]$ & Craig Rogers & ISI & RogerseISI.EDU \\
\hline [CO16] & Chris Olson & FIRENET & --- none--- \\
\hline$[\mathrm{CP} 10]$ & Craig Partridge & $\mathrm{BBN}$ & craig@BBN.COM \\
\hline [CSTACY] & Christopher stacy & Palladian & CStacy@AI.AI.MIT.EDU \\
\hline [CWH3 ] & Craig W. Hunt & NBS & CRAIG@CAM-VAX.ARPA \\
\hline$[\mathrm{CYH}]$ & Chien Y. Huang & \multicolumn{2}{|c|}{$\begin{array}{l}\text { PRINCETON } \\
\quad 6026959 \% \text { PUCC.BITNET@WISCVM.WISC.EDU }\end{array}$} \\
\hline [DAT 4$]$ & Doug A. Thomae & HARRIS & --- none--- \\
\hline [DAVE] & David Roode & \multicolumn{2}{|c|}{ Intellicorp Roode@BIONET-20.ARPA } \\
\hline [DB14] & Dave Borman & CRAY & dab@UMN-REI-UC.ARPA \\
\hline [DB28 ] & Dave Bullard & \multicolumn{2}{|c|}{$\begin{array}{l}\text { CLEMSON } \\
\text { dave\%clemson.bitnet@WISCVM.WISC.EDU }\end{array}$} \\
\hline [DB35 $]$ & Danny Branis & \multicolumn{2}{|c|}{$\begin{array}{l}\text { HUJ } \\
\text { dannyISRAEL.CSNET@RELAY.CS.NET }\end{array}$} \\
\hline [DB97 ] & Dave Bergum & \multicolumn{2}{|c|}{ HONEYWELL bergum@HI-MULTICS.ARPA } \\
\hline$[\mathrm{DB} 150]$ & David Bloom & \multicolumn{2}{|c|}{ RUTGERS } \\
\hline$[\mathrm{DBJ}]$ & David B. Johnson & DRILLTECH & DBJ@RICE.EDU \\
\hline [DC99] & David Chan & CIT & chan@BEK-MC.CALTECH.EDU \\
\hline$[\mathrm{DC} 126]$ & Dick Cogger & $\mathrm{CU}$ & \\
\hline & & \multicolumn{2}{|c|}{ rhx $\%$ cornellc.bitnet@WISCVM.WISC.EDU } \\
\hline [DCMW ] & David C. M. Wood & $\mathrm{CU}$ & DCMWOOD@COLO.COLORADO.EDU \\
\hline$[\mathrm{DD} 11]$ & Don Deal & GATECH & DONQPYR.GATECH.EDU \\
\hline [DD 41$]$ & Dan DeGrossa & NSWSES & NSWSES@DDN2.ARPA \\
\hline$[\mathrm{DD} 47]$ & Diane Donaldson & USDOL & ANDIE@CVL. UMD . EDU \\
\hline$[\mathrm{DDC} 1]$ & David Clark & MIT & DClark@MIT-MULTICS.ARPA \\
\hline [DE6] & Deborah Estrin & USC & Estrin@USC-CSEB.USC.EDU \\
\hline [DEC1 $]$ & Douglas E. Comer & PURDUE & DEC@PURDUE.EDU \\
\hline$[\mathrm{DF} 71]$ & David Fordyce & TI & fordyce $\%$ t-csl@RELAY.CS.NET \\
\hline [DFH2 ] & Dan Hocking & AIRMICS & DHOCKING@ISI.EDU \\
\hline [DGH13 ] & Donald G. Hirsh & WU & wucs 1 ! wucs 2 ! don@seismo. CSS.GOV \\
\hline [DGT6] & David G. Taylor & INFERENCE & --- none--- \\
\hline [DH17] & Douglas Hirsch & $\mathrm{BBN}$ & dhirsch@CCS.BBN.COM \\
\hline [DH23 ] & David Hayes & HQDA & dshayes@SMOKE.BRL.MIL \\
\hline [DH30 ] & Doc Hayes & ARMY & $\mathrm{ns}-\mathrm{ddn} @ \mathrm{DDN} 2 . \mathrm{ARPA}$ \\
\hline$[\mathrm{DJF}]$ & David J. Farber & UDEL & Farber@HUEY. UDEL. EDU \\
\hline [DJG2 ] & Daniel J. Grim & UDEL & grimeHUEY.UDEL.EDU \\
\hline [DJV1 ] & Darrel J. Van Buer & SDC & vanbuer@ECLA.USC.EDU \\
\hline [DK2 ] & Dean B. Krafft & CORNELL & Dean@GVAX. CS. CORNELL. EDU \\
\hline [DK5 ] & Diana Kirby & SAS & --- none--- \\
\hline
\end{tabular}




\begin{tabular}{|c|c|c|c|}
\hline$[\mathrm{DK} 66]$ & Doug Konkin & \multicolumn{2}{|c|}{$\begin{array}{l}\text { ARC } \\
\text { doug\%noah. arc. cdn\%ubc. csnet@RELAY.CS.NET }\end{array}$} \\
\hline [DLM1 ] & David Mills & LINKABIT & Mills@HUEY.UDEL.EDU \\
\hline [DLM34 ] & David L. Merrifield & $\mathrm{UAF}$ & --- none--- \\
\hline [DM2 7 ] & Doug McCallum & ISC & mccallumeICO.ISC.COM \\
\hline [DM1 47$]$ & Dan Morales & HONEYWELL & --- none--- \\
\hline [DMK16] & Debra M. Kukanich & $\mathrm{ETA}$ & --- none--- \\
\hline [DMK18 ] & David M. Keirsey & HUGHES & KEIRSEY@ECLA.USC.EDU \\
\hline [DN22 ] & David Novotny & \multicolumn{2}{|c|}{$\begin{array}{l}\text { HARC } \\
\text { DMN8 672\%TAMVENUS. BITNET@WISCVM.WISC.EDU }\end{array}$} \\
\hline [DN32 ] & Dave Nordlund & \multicolumn{2}{|c|}{$\begin{array}{l}\text { UK } \\
\text { NORDLUNDUKANVM. BITNET@WISCVM.WISC.EDU }\end{array}$} \\
\hline [DO26 ] & Dennis O'Reilly & $\mathrm{UBC}$ & --- none--- \\
\hline [DO27 ] & David Oliver & ANSA & ANSA $\circ A L V E Y$. UK@CS. UCL.AC.UK \\
\hline [DP 71 ] & David Palus & $\mathrm{NEC}$ & --- none--- \\
\hline [DGT 6 ] & Dave Taylor & INFERENCE & --- none--- \\
\hline$[\mathrm{DR} 71]$ & Duane Rettig & AMDAHL & --- none--- \\
\hline$[\mathrm{DS} 85]$ & Dale Smith & \multicolumn{2}{|c|}{ dsmith\%oregon2.bitnet@WISCVM.WISC.EDU } \\
\hline$[\mathrm{DS} 160]$ & Don Scelza & PERQ & --- none--- \\
\hline$[\mathrm{DSR}]$ & Dale Russell & UNISYS & dsr@ JOVE. CAM. UNISYS. COM \\
\hline [DSW ] & Dan Whelan & CALTECH & Dan@CIT-20.CALTECH.EDU \\
\hline [DT50 ] & Daniel Trinkle & PURDUE & trinkle@PURDUE.EDU \\
\hline$[\mathrm{DTH}]$ & David T. Hsu & WEDGE & hsu@ENEEVAX. UMD . EDU \\
\hline [DW93 ] & David Watson & COGNITION & david@DANDELION.CI.COM \\
\hline [DW96] & David Walker & \multicolumn{2}{|c|}{ DHWalker\%ucivmsa.bitnet@WISCVM.WISC.EDU } \\
\hline$[\mathrm{EC} 5]$ & Ed Cain & DCEC & cain@EDN-UNIX.ARPA \\
\hline [ED38 ] & Ed DeHart & TARTAN & DEHART@TL-20B.ARPA \\
\hline [EEW6] & Ernest Woodward & NU & ernie@NORTHWESTERN.ARPA \\
\hline [EF 5 ] & Ed Franceschini & NYU & Franceschini@NYU.ARPA \\
\hline [EHH4 ] & Eddie H. Hunter & UGA & --- none--- \\
\hline [EJN1] & Eric J. Norman & WISC & EJNorman@UNIX2.MACC.WISC.EDU \\
\hline [EK18] & Edwin King & SRI & King@SPAM.ISTC.SRI.COM \\
\hline$[\mathrm{EPA}]$ & Eric Allman & BLI & eric@UCBVAX.Berkeley.EDU \\
\hline$[\mathrm{ERC} 1]$ & Eric R. Crane & ACCENT & Eric.CranedC.CS.CMU.EDU \\
\hline [ERK3 ] & Edward Kozel & SRI & Kozel@SPAM.ISTC.SRI.COM \\
\hline [EY5 ] & Elaine Yamin & ATT & --- none--- \\
\hline [EZ3 ] & Edward Zawacki & UIC & \\
\hline & & \multicolumn{2}{|c|}{ u17375\%uicvm.bitnet@WISCVM.WISC.EDU } \\
\hline$[F A S]$ & Fred Segovich & GSWD & fred@GSWD-VMS.ARPA \\
\hline$[\mathrm{FCH}]$ & Franklin C. Holtry & $\mathrm{UCD}$ & --- none--- \\
\hline [FD18 $]$ & F. de Kruijf & DUTNET & FREEK $\%$ DUTRUN. UUCP @ seismo. CSS.GOV \\
\hline [F JK2 ] & Frank J. Kastenholz & SPARTACUS & KODINSKY@MIT-MULTICS.ARPA \\
\hline [FJS3 ] & F. Jeffery Schmidt & $\mathrm{CDA}$ & JSCHMIDT.CDA@AMC-HQ. ARPA \\
\hline [FMA1 ] & Frederick M. Avolio & DECUAC & Avolio@DECUAC.DEC.COM \\
\hline [FS37] & Frank Solensky & PRIME & --- none--- \\
\hline [FW1 7] & Frederic Wendling & $\mathrm{NSF}$ & FWENDLING@NOTE.NSF . GOV \\
\hline
\end{tabular}




\begin{tabular}{|c|c|c|c|}
\hline [FWD ] & Wolfgang J. Dyner & USAREUR & --- none--- \\
\hline [GAA $]$ & Glenn A. Adams, Jr. & $\mathrm{MIT} / \mathrm{LL}$ & glenneXN.LL.MIT.EDU \\
\hline [GAL5 ] & Guillermo A. Loyola & IBM & Loyola\%ibm-sj@RELAY.CS.NET \\
\hline [GB 7 ] & Gerd Beling & DFVLR & GBELING@ISI.EDU \\
\hline [GB 43 ] & George Broomell & $\begin{array}{l}\text { UKY } \\
\text { UKT101\%UI }\end{array}$ & KCC.BITNET@WISCVM.WISC.EDU \\
\hline [GBR ] & G. Brendan Reilly & WHARTON & Reilly@WHARTON.ARPA \\
\hline$[\mathrm{GC}]$ & Graham Campbell & BNL & gc@BNL. ARPA \\
\hline$[\mathrm{GEOFF}]$ & Geoffrey Mulligan & USAF & GEOFF@USAFA.ARPA \\
\hline [GG11 ] & George Goble & PURDUE & ghg@EE.ECN.PURDUE.EDU \\
\hline [ GG 43 ] & Gary Gagnon & $\mathrm{CSC}$ & GAGNON@AF SC-HQ.ARPA \\
\hline [GGB2 ] & Geoffrey G. Baehr & TRW & geoffb@TRWIND.TRW.COM \\
\hline [GH29] & Gregory Hidley & UCSD & hidley@SDCSVAX.UCSD.EDU \\
\hline$[\mathrm{GIH}]$ & Glenn I. Hastie II & SRI & Hastie@SPAM.ISTC.SRI.COM \\
\hline [GKN1 ] & Gerard K. Newman & $\mathrm{SDSC}$ & gkn@SDSC-SDS.ARPA \\
\hline [GL41] & Gunnar Lindberg & $\mathrm{CTH}$ & --- none--- \\
\hline$[\mathrm{GLD}]$ & Geraldine L. Durant & LL & DURANT@LL.ARPA \\
\hline [GLH5 ] & Gavin L. Hamphill & DREA & Hemphill@DREA-XX.ARPA \\
\hline [GM3 4 ] & Gaylord Miyata & $\begin{array}{l}\text { Goldhill } \\
\text { Miyata\%o }\end{array}$ & .ai.mit.edu@XX.LCS.MIT.EDU \\
\hline [GP 56 ] & Gottfried Petschl & TUNET & --- none--- \\
\hline [GP L1] & Gene LeClair & Pentagon & GENE@OPTIMIS-PENT.ARPA \\
\hline [GR2 6] & Georg Richter & RU & --- none--- \\
\hline$[\mathrm{GTA}]$ & Guy T. Almes & RICE & almes@RICE.EDU \\
\hline [ GW22 ] & Grant Weiler & UTAH & Weiler@CS.UTAH.EDU \\
\hline [ GW 40 ] & Gary Wallace & $\begin{array}{l}\text { UMASS } \\
\text { gary } \% \text { uma }\end{array}$ & S. Csnet@RELAY.CS.NET \\
\hline [ GW4 9] & George Ward & Motorola & --- none--- \\
\hline [ $\mathrm{HC} 2]$ & Haesoon Cho & $\begin{array}{l}\text { KAIST } \\
\text { hscho\%ka }\end{array}$ & st. csnet@RELAY.CS.NET \\
\hline [ $\mathrm{HC} 24]$ & Ho Chen & INTEL & --- none--- \\
\hline [ HDW2 ] & Howard Wactlar & $\mathrm{CMU}$ & Howard. Wactlar@A. CS. CMU.EDU \\
\hline [HGH1] & Harry G. Heard & JVNC & HEARD@AMES-VMSB . ARPA \\
\hline [HM38 ] & Hirohide Mikami & $\begin{array}{l}\text { NTT } \\
\text { mikami }\end{array}$ & $t-20 @$ SUMEX-AIM.STANFORD.EDU \\
\hline [ HML1] & H. Morrow Long & YALE & long-morrow@YALE.ARPA \\
\hline [HN3 ] & Heinz Naef & CIBA & mcvax!cgcha! whna@seismo.CSS.GOV \\
\hline [HT12] & Henry Tam & $\begin{array}{l}\text { NYTEL } \\
\quad \text { rmay } \% c\end{array}$ & ornelld.bitnet@ JADE. Berkeley.EDU \\
\hline [HWB ] & Hans-Werner Braun & MICHIGAN & HWB@MCR. UMICH.EDU \\
\hline [HWP 2 ] & Henry W. Poor & UM & poororsmas. span@ JPL-VLSI . ARPA \\
\hline$[\mathrm{IRN}]$ & Isaac R. Nassi & ENCORE & NASS I @MULT IMAX . ARPA \\
\hline [ IW5 ] & Ira Winston & UPENN & Ira@CIS. UPENN.EDU \\
\hline$[\mathrm{JA}]$ & Jaap Akkerhuis & WCW & jaap@MOUTON.ARPA \\
\hline$[\mathrm{JA1}]$ & Jules P. Aronson & NLM & Aronson@MCS.NLM.NIH.GOV \\
\hline [ JAG3 ] & Jeff Gumpf & CWRU & G.Gumpf@CS.COLUMBIA.EDU \\
\hline [ JAJ1 7 ] & James Jokl & UVA & --- none--- \\
\hline [ JB1 13 ] & Jerome Bennett & NASA & bennett@MPP.GSFC.NASA.GOV \\
\hline
\end{tabular}




\begin{tabular}{|c|c|c|c|}
\hline [ JB188 ] & Josef Burger & WISC & bolo@SPOOL.WISC.EDU \\
\hline [ JB218] & Jim Blondeau & TEK & \\
\hline & & \multicolumn{2}{|c|}{ jbb\%tektools.tek. csnet@RELAY.CS.NET } \\
\hline [ JBC2 ] & John B. Chambers & UT & jbc@SALLY.UTEXAS.EDU \\
\hline$[\mathrm{JBP}]$ & Jon Postel & ISI & Postel@ISI.EDU \\
\hline [ JBW1 ] & Joseph Walters, Jr. & $\mathrm{BBN}$ & JWalters@CCX.BBN.COM \\
\hline$[\mathrm{JC} 11]$ & Jim Clifford & LANL & jrc@LANL.GOV \\
\hline$[\mathrm{JC} 106]$ & Joel Conklin & $\mathrm{GE}$ & Conklin@GE-CRD.ARPA \\
\hline [ JCB42 ] & Jay C. Bergeron & FACTRON & --- none--- \\
\hline [ JCH1 7 ] & Jeffrey Honig & CLARKSON & JCH@OMNIGATE.CLARKSON. EDU \\
\hline$[\mathrm{JCN} 2]$ & John C. Nunn & NBS & NUNN@NBS-VMS . ARPA \\
\hline [ JCW12] & James C. Woodard & ROCKWELL & --- none--- \\
\hline [ JD27 ] & John Doyle & CIT & doyle@CSVAX.CALTECH.EDU \\
\hline [ JDC20 ] & Jeffrey D. Case & \multicolumn{2}{|l|}{$\begin{array}{l}\text { UTK } \\
\text { jdc }\end{array}$} \\
\hline [ JDG ] & Jim Guyton & RAND & guyton@RAND-UNIX.ARPA \\
\hline [ JEE4] & Jan Ellison & GTE & --- none--- \\
\hline$[\mathrm{JEM}]$ & Jim Mathis & SRI & Mathis@KL.SRI.COM \\
\hline [ JF 7 7 ] & Jim Fallon & MACOM & JFALLON@MACOMW. ARPA \\
\hline [ JGD1 ] & Joseph G. Deck & WU & \\
\hline \multicolumn{4}{|c|}{ deckvax.weslyn\%wesleyan.bitnet@WISCVM.WISC.EDU } \\
\hline [ JH18 ] & Jean Huens & KULEUVEN & prlb2!kulcs! jean@seismo.CSS.GOV \\
\hline [ JH92 ] & Jack Hahn & \multicolumn{2}{|c|}{$\begin{array}{l}\text { UMDC } \\
\text { hahn\%umdc.bitnet@WISCVM.WISC.EDU }\end{array}$} \\
\hline [ JH141] & Juha Heinanen & \multicolumn{2}{|c|}{ FINLAND } \\
\hline & & \multicolumn{2}{|c|}{ I-TECHNICAL-CONTACTTUT. UUCP A seismo. CSS.GOV } \\
\hline [ JH1 55 ] & Jeff Hayward & \multicolumn{2}{|c|}{$\mathrm{UH}$} \\
\hline & & \multicolumn{2}{|r|}{ UCC1UHVAX1.BITNET@WISCVM.WISC.EDU } \\
\hline [ JHH 8 ] & James H. Haynes & UCSC & ucscC! haynes@UCBVAX. Berkeley.EDU \\
\hline [ JJ48 $]$ & Jeffrey Jongeward & \multicolumn{2}{|c|}{ ssc-vax!root@BEAVER.CS.WASHINGTON. EDU } \\
\hline [ JJD12 ] & Jeff Diehl & USAF & $\mathrm{XQR}-\mathrm{SPCD} \mathrm{AFCC}-4 . \mathrm{ARPA}$ \\
\hline$[\mathrm{JK} 7]$ & Jim Koda & ISI & Koda@ISI.EDU \\
\hline [ JKR1 ] & Joyce K. Reynolds & ISI & JKREYNOLDS@ISI.EDU \\
\hline [ JL15] & Jay Lepreau & $\mathrm{UTAH}$ & Lepreau@CS.UTAH.EDU \\
\hline [ JLM2 3 ] & John L. Mills & HONEYWELL & Mills@BCO-MULTICS.ARPA \\
\hline [ JLR4 ] & John Romkey & FTPSW & Romkey@BORAX.LCS.MIT.EDU \\
\hline [ JLS4 5 ] & John L. Sloan & WSU & jsloan\%wright. csnet@RELAY.CS.NET \\
\hline [ JM2 8 ] & John W. Milton & $\mathrm{DCA}$ & DCAB650@DDN1.ARPA \\
\hline [ JM60 ] & Jim McCollum & $\mathrm{DEC}$ & mCCOllum@MARLBORO.DEC.COM \\
\hline [ JM2 78 ] & Jin Mazumdar & FREDONIA & --- none--- \\
\hline [ JM2 92 ] & Jun Murai & UTOKYO & jun\% japan. csnet@RELAY.CS.NET \\
\hline [ JM303] & John Moorfoot & DEAKINET & jgm\%charlie.oz@seismo.CSS.GOV \\
\hline [ JM304 ] & Jim McClurg & Sperry & --- none--- \\
\hline [ JMA1 6 ] & James M. Adams & $\mathrm{MACOM}$ & ADAMS@MACOMW. ARPA \\
\hline [ JMR ] & John M. Rushby & SRI & Rushby@DOCKMASTER.ARPA \\
\hline [ JN4 0 ] & John Noble & VCU & --- none--- \\
\hline$[\mathrm{JN} 47]$ & Jerry Nerbovig & WELLSNET & --- none--- \\
\hline
\end{tabular}




\begin{tabular}{|c|c|c|c|}
\hline [ JNL1 ] & John Larson & XEROX & jlarson.pa@XEROX.COM \\
\hline [ JO5 4 ] & John O'Connor & NYTEL & --- none--- \\
\hline [ JOG ] & John O. Gartley & \multirow{2}{*}{\multicolumn{2}{|c|}{$\begin{array}{l}\text { ALCOA } \\
\quad \text { gartley\%atc.alcoa.com@RELAY.CS.NET }\end{array}$}} \\
\hline & & & \\
\hline$[\mathrm{JOO}]$ & James O. Ostlund & SALKNET & ostlund@SALK-ADM.SDSC.EDU \\
\hline [ JPS17 ] & John P. Stoneback & \multicolumn{2}{|c|}{$\begin{array}{l}\text { MORAVIAN } \\
\text { allegra!mc70! stonebac@seismo.CSS.GOV }\end{array}$} \\
\hline [ JR15 ] & John Rhodes & LOGNET & JRhodes@LOGNET2 . ARPA \\
\hline [ JR1 7 ] & John L. Robinson & CANADA & Robinson@DMC-CRC.ARPA \\
\hline [ JRL3 ] & John LoVerso & \multicolumn{2}{|c|}{ LoVerso\%buffalo. csnet@RELAY.CS.NET } \\
\hline [ JRR14 ] & Joe Ragland & TUCC & TUCJRR@TUCC.TUCC.EDU \\
\hline [ JS28 ] & John A. Shriver & PROTEON & JAS@PROTEON . COM \\
\hline [ JS38 ] & Joseph Sventek & LBL & JSSventek@LBL.ARPA \\
\hline [ JS81 ] & Jeff Smith & PURDUE & aat@J.CC.PURDUE.EDU \\
\hline [ JS171] & Jerry scott & TWG & --- none--- \\
\hline [ JS268 ] & J. Simonetti & SUNY & --- none--- \\
\hline [ JS283 ] & Jack Schwartz & ARPA & jschwartz@ISI.EDU \\
\hline [ JSG5 ] & Jon Goodridge & $\mathrm{BBN}$ & jsgeCCM.BBN.COM \\
\hline [ JSS 4$]$ & Jayant S. Sabnis & SRA & sabnix\%onrl.decnet@NRL.ARPA \\
\hline [ JSY2] & Jeffrey S. Yaplee & BOEING & --- none--- \\
\hline [ JTE2 ] & James T. Ellis & PSCNET & ellis@MORGUL.PSC.EDU \\
\hline [ JTN ] & John T. Nelson & $\mathrm{ADS}$ & jtn@ADS.ARPA \\
\hline [ JW4 7 ] & John Wobus & \multicolumn{2}{|l|}{$\begin{array}{l}\text { SUCNS } \\
\text { JMWobus }\end{array}$} \\
\hline [ JW136 ] & James D. White & UOKNOR & jdw@UOKUCS. UOKNOR.EDU \\
\hline [ JW1 56 ] & John Wray & RSRE & JCW2\%RSRE@CS.UCL.AC.UK \\
\hline [ JWO1 ] & James W. O'Toole & UMD & james@MIMSY . UMD . EDU \\
\hline [ JY11 ] & Joe Yancone & USARMY & Yancone@CRDEC.ARPA \\
\hline [KA4 ] & Karl Auerbach & EP ILOGUE & auerbach@CSL.SRI. COM \\
\hline [KB 60$]$ & Karl Braun & $\mathrm{DRI}$ & --- none--- \\
\hline$[\mathrm{KBC}]$ & Kevin B. Casey & \multicolumn{2}{|l|}{ Gallaudet } \\
\hline$[\mathrm{KC} 8]$ & Ken Chen & \multicolumn{2}{|c|}{ Perceptronics $\quad---$ none--- } \\
\hline [ KCM2 ] & Kelly C. McDonald & \multicolumn{2}{|c|}{ BYU } \\
\hline [KDM5 ] & Keith D. Miller & TASC & --- none--- \\
\hline [KDZ ] & Kurt D. Zeilenga & UNM & zeilenga@HC.DSPO.GOV \\
\hline [KFD ] & Ken Dove & AIDS & kfd@ADS.ARPA \\
\hline$[\mathrm{KHJ}]$ & Karen Jobes & \multicolumn{2}{|c|}{$\begin{array}{l}\text { IASNET } \\
\text { jobes\%iassns.bitnet@WISCVM.WISC.EDU }\end{array}$} \\
\hline [KL31] & Kathleen Lamb & CSM & klamb\%csm9a@COLO. COLORADO. EDU \\
\hline [ KMC3 ] & Kenneth M. Crepea & SRI & Crepea@SACFS.ARPA \\
\hline [ KMH 8 ] & Ken Hays & FSU & haysfsu.mfenet@NMFECC.ARPA \\
\hline [KO11] & Kevin $O^{\prime}$ Keefe & HAZELTINE & Hazeltine@ISI.EDU \\
\hline [KR9] & J. Keven Rohan & FORD & JJKKRR@FORD-COS1. ARPA \\
\hline [KS 62$]$ & Kathy Simpson & OSU & --- none--- \\
\hline$[\mathrm{KSL}]$ & Kirk Lougheed & CISCO & Lougheed@KL.SRI.COM \\
\hline
\end{tabular}




\begin{tabular}{|c|c|c|c|}
\hline [KTP ] & Kenneth T. Pogran & BBN & Pogran@CCQ.BBN.COM \\
\hline [KW2 ] & Keith T. Wescourt & FMC & WESCOURT@CEL.FMC.COM \\
\hline [ LAM1 ] & Louis A. Mamakos & UMD & louie@TRANTOR. UMD.EDU \\
\hline [LB16] & Liudvikas Bukys & ROCHESTER & Bukys@CS.ROCHESTER.EDU \\
\hline$[\mathrm{LCN}]$ & Lou Nelson & AEROSPACE & Lou@AEROSPACE.AERO.ORG \\
\hline$[\mathrm{LCS}]$ & Lou Schreier & SRI & Schreier@SPAM.ISTC.SRI.COM \\
\hline$[\mathrm{LFO}]$ & Luis F. Ortiz & YALE & Ortiz-Luis@YALE.ARPA \\
\hline [LJR5 ] & Louis J. Romero & MMDA & --- none--- \\
\hline [LL53 ] & Leo Lanzillo & $\mathrm{BBN}$ & leo@SH.CS.NET \\
\hline [LL56 ] & Len Lattanzi & SENTRY & LATTANZI@SUMEX-AIM.STANFORD . EDU \\
\hline [ LM62 ] & Landy Manderson & $\begin{array}{l}\text { UAB } \\
\text { usts } 034 \% \text { u }\end{array}$ & dabtucc.bitnet@WISCVM.WISC.EDU \\
\hline [ LM8 8 ] & Lee McLoughlin & ICNET & lm jmঃdoc.ic.ac.uk@CS.UCL.AC.UK \\
\hline [ LOU ] & Lou Salkind & NYU & Salkind@NYU.ARPA \\
\hline$[\mathrm{LPM}]$ & Leslie P. Michelson & UMDNJ & --- none--- \\
\hline$[\mathrm{LRB}]$ & Larry Bierma & NPRDC & Bierma@NPRDC.ARPA \\
\hline$[\mathrm{LRC} 7]$ & Larry R. Custead & $\begin{array}{l}\text { USASK } \\
\text { custea }\end{array}$ & ad\%sask.bitnet@WISCVM.WISC.EDU \\
\hline [LRR1 ] & Lawrence Rogers & Princeton & Irr@PRINCETON.EDU \\
\hline$[\mathrm{LS} 103]$ & Leon Schilmoeller & $3 \mathrm{M}$ & --- none--- \\
\hline [LT28 ] & Larry Taylor & $\begin{array}{l}\text { UIOWA } \\
\text { BPTLCTPB\% }\end{array}$ & JUIAMVS.BITNET@WISCVM.WISC.EDU \\
\hline [ LW2 6 ] & Linda Winkler & $\begin{array}{l}\text { ARGONNE } \\
\text { B32357\%AN }\end{array}$ & JLVM.BITNET@WISCVM.WISC.EDU \\
\hline$[\mathrm{LWR}]$ & Larry Robinson & LLNL & lwr@MORDOR.S1.GOV \\
\hline$[\mathrm{MA}]$ & Mike Accetta & $\mathrm{CMU}$ & MIKE.ACCETTA@A.CS.CMU.EDU \\
\hline [MA2 4 ] & Melanie Anderson & $\begin{array}{l}\text { UI } \\
\text { melanie } \% n\end{array}$ & ICsavmsa.bitnet@WISCVM.WISC.EDU \\
\hline [MA 54 ] & Manny Allegue & TRINCOLL & --- none--- \\
\hline [MAB 4 ] & Mark Brown & USC & Mark@OBERON.USC.EDU \\
\hline [MAJ1 $]$ & M.A. Johnson & CAMBRIDGE & --- none--- \\
\hline$[\mathrm{MB}]$ & Michael Brescia & $\mathrm{BBN}$ & Brescia@CCV.BBN.COM \\
\hline [MB2 6] & Mike Brzustowicz & $\mathrm{ADS}$ & mab@ADS.ARPA \\
\hline [MB31] & Michael Bereschinsky & USARMY & Bereschinsky@ISI.EDU \\
\hline [MC17] & Matt Crawford & UCHICAGO & Crawford@ANL-MCS.ARPA \\
\hline [MC 6 5 ] & Michael Corn & NYNEX & --- none--- \\
\hline [MCA1 $]$ & Mary Crocombe Akers & $\mathrm{BBN}$ & makers@BBN.COM \\
\hline$[\mathrm{MDC}]$ & Martin D. Connor & MIT AI & mdc@BHT.AI.MIT.EDU \\
\hline [MF 31] & Martin J. Fouts & NASA-AMES & fouts@AMES-NAS.ARPA \\
\hline [MF 52 2 & Michael Figg & LOCKHEED & mikefigg@AIVAX. LAD. COM \\
\hline [MG58 ] & Mike Gilbert & SLI & MBALLENTINE@ISI.EDU \\
\hline \multirow[t]{2}{*}{ [MH 82 ] } & Mark Horton & $\mathrm{ATT}$ & \\
\hline & \multicolumn{3}{|c|}{ cbosgd!cbpavo.mis.oh.att.com!mark@seismo.CSS.GOV } \\
\hline [ $\mathrm{MH} 98$ ] & Michael Hrybyk & JHU & hrybyk@HOPKINS-EECS-BRAVO.ARPA \\
\hline [MHG ] & Alma Grijalva & UARIZ & USARCCO@SIMTEL20.ARPA \\
\hline [MJM2 ] & Mike Muuss & BRL & Mike@BRL.ARPA \\
\hline$[\mathrm{MJO} 4]$ & Mike O'Connor & SPACECOM & oconnor@SCCGATE.SCC.COM \\
\hline [MK1 7 ] & Mike Karels & BERKELEY & Karels@uCBVAX.Berkeley.EDU \\
\hline
\end{tabular}




\begin{tabular}{|c|c|c|c|}
\hline [MK38 ] & Mark Kowitz & ROCKEFELLI & ¿R Mark@ROCKEFELLER. ARPA \\
\hline [MK 68 ] & Michael Kazar & $\mathrm{CMU}$ & Mike.Kazar@K.CS.CMU.EDU \\
\hline [MKP 2$]$ & Michael K. Peterson & HUGHES & scgvaxd!mkp@CSVAX. CALTECH.EDU \\
\hline [ML 62 ] & Michael Levine & CMU & Levine@A.PSY.CMU.EDU \\
\hline$[\mathrm{MLC}]$ & Mike Corrigan & $\mathrm{DDN}$ & Corrigan@DDN3.ARPA \\
\hline [MM135 ] & M. Mills & GTE & --- none--- \\
\hline [MM1 47$]$ & Mark Meyer & UN & \\
\hline [MM1 49] & Mark Miller & $\begin{array}{l}\operatorname{mark} \% \\
\text { LEHIGH } \\
\quad \text { lumm } \%\end{array}$ & $\begin{array}{l}\text { anlcdc3.bitnet@WISCVM.WISC.EDU } \\
\text { ehiibm1.bitnet@WISCVM.WISC.EDU }\end{array}$ \\
\hline [MMH5 ] & Martin Hayman & Symbolics & --- none--- \\
\hline [MMM3 ] & Michael McDonnell & USAETL & Mike@ETL.ARPA \\
\hline [MMM2 5 ] & Marc M. Meilleur & COINS & COINS@ISI.EDU \\
\hline [MO14] & Michele Olivant & JHU & Olivant@HAWAII-EMH.ARPA \\
\hline [MP 20 ] & Michel Perras & NUSC & Perras@NUSC-ADA.ARPA \\
\hline$[\mathrm{MPM}]$ & M. Preston Mullen & NRL & mullen@NRL-CSS.ARPA \\
\hline [MR2 9] & Mike Russell & BROWN & --- none--- \\
\hline [MR78 ] & Michael Rotert & LINK & ZORN $\%$ GERMANY. CSNET@RELAY. CS.NET \\
\hline [MS 9 ] & Martin Schoffstall & RPI & schoff@CSV.RPI.EDU \\
\hline [MS 22 ] & Mark Starner & Unisys & starnereBIGBURD.PRC.UNISYS.COM \\
\hline [MS101] & Michael Szymendera & CANISIUS & mikeyocanisius.edu@RELAY.CS.NET \\
\hline$[\mathrm{MS} 171]$ & Marc Shapiro & INRIA & Marc.Shapiro@C.CS.CMU.EDU \\
\hline [MS172] & Marina Simonians & RDL & --- none--- \\
\hline$[\mathrm{MSA} 1]$ & Mats Andersson & Sweden & --- none--- \\
\hline [MSM1 ] & Milo S. Medin & AMES & medin@AMES.ARPA \\
\hline [MSP 1$]$ & Mark St. Paul & NMSU & stpaul\%nmsu. csnet@RELAY.CS.NET \\
\hline [MT1 ] & Michael Tharenos & IBM & postmaster@IBM.COM \\
\hline$[\mathrm{MTR}]$ & Marshall Rose & NRTC & MROse@GREMLIN.NRTC.NORTHROP. COM \\
\hline$[\mathrm{MV} 24]$ & Mark Vasoll & $\begin{array}{l}\text { OKSTATE } \\
\text { vasoll } \% \text { a }\end{array}$ & Cs.okstate.edu@RELAY.CS.NET \\
\hline [MW49] & Mark Waldschmidt & SAIC & --- none--- \\
\hline [MWS10 ] & Michael Stalnaker & FAI & MIKE@NRL-SSD.ARPA \\
\hline$[\mathrm{NAL}]$ & Neil Lann & LLL & NAL@LLL-TIS-B.ARPA \\
\hline [NC3 ] & J. Noel Chiappa & MIT & JNC@XX.LCS.MIT.EDU \\
\hline [NG ] & Neil Gower & ROCKWELL & GOWER@ISI.EDU \\
\hline [NH2 ] & Nat Howard & IM & nrh@FLASH . BELLCORE. COM \\
\hline$[\mathrm{NIC}]$ & Net Info Center & SRI & Hostmaster@SRI-NIC.ARPA \\
\hline [NMM ] & Mike Minnich & UDELEE & MMinnich@HUEY. UDEL.EDU \\
\hline [NSE] & Nayel el-Shafei & PRIME & Shafeiomit-oz@AI.AI.MIT.EDU \\
\hline [NT12] & Neil Todd & IST & mcvax! ist! neileseismo.CSS.GOV \\
\hline [NT13] & Nigel Titley & BTRL & \\
\hline & & $\mathrm{mcV}$ & ax!btnix!titley@seismo.CSS.GOV \\
\hline [OG4 ] & $\begin{array}{l}\text { Olivier Gremont } \\
\text { mcvax! }\end{array}$ & INRIA & 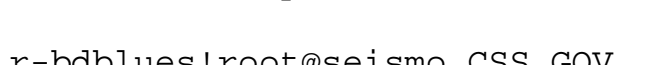 \\
\hline [PA5 ] & Philip Almquist & STANFORD & Almquist@SCORE.STANFORD.EDU \\
\hline [PAM6] & Paul McNabb & $\mathrm{RICE}$ & pam@PURDUE.EDU \\
\hline [PAP 4 ] & Philip Prindeville & FTPSW & philipp@MC.LCS.MIT.EDU \\
\hline$[\mathrm{PB} 40]$ & Phil Bowden & $\mathrm{VA}-\mathrm{TECH}$ & \\
\hline
\end{tabular}




\begin{tabular}{|c|c|c|c|}
\hline \multicolumn{4}{|c|}{ BOWDEN!VTVM1.BITNET@WISCVM.WISC.EDU } \\
\hline$[\mathrm{PB} 67]$ & Pat Boyle & UBC & boyle\%ubc.csnet@RELAY.CS.NET \\
\hline$[\mathrm{PC} 55]$ & Phyliss Charlton & MACOM & --- none--- \\
\hline [PD 39] & Pete Delaney & $\mathrm{ECRC}$ & \\
\hline \multicolumn{4}{|c|}{ pete\%ecrcvax. uucp \%germany. csnet@RELAY.CS.NET } \\
\hline [PDB5 $]$ & Patrick D. Barron & $\mathrm{CMU}$ & Pdb@SEI.CMU.EDU \\
\hline$[\mathrm{PFK}]$ & Peter F. King & NEXT & king\%next. com@RELAY.CS.NET \\
\hline [PFS2] & Paul Sass & CECOM & Sass@ISI.EDU \\
\hline [PGA1 ] & Phillip G. Apley & BITSTREAM & --- none--- \\
\hline$[\mathrm{PGM}]$ & Paul G. Milazzo & RICE & Milazzo@RICE.EDU \\
\hline [PH4 5 ] & Peter Ho & HAC & ho\%athena. hac. com@OBERSON. USC. EDU \\
\hline$[\mathrm{PK}]$ & Peter Kirstein & UCL & Kirstein@ISI.EDU \\
\hline [PK19] & Penny Karr & $\mathrm{BBN}$ & pkarr@BBN.COM \\
\hline [PK28] & Philip R. Karn, Jr. & $\mathrm{BCR}$ & Karn@FLASH.BELLCORE.COM \\
\hline [PKH1 ] & Paul Hyder & UCSB & UCSBCSL ! HYDER@UCBVAX . Berkeley . EDU \\
\hline [PLH 8 ] & Paula L. Haymon & $\mathrm{UT}$ & --- none--- \\
\hline [PM4 ] & Paul Martin & SRI & PMartin@KL.SRI.COM \\
\hline [PM37 ] & Phyllis Melvin & BOEING & phyllis@BOEING.COM \\
\hline [PM72] & Paul Mies & GMD & --- none--- \\
\hline [PMH3 ] & P. M. Henderson & WACHOVIA & --- none--- \\
\hline$[\mathrm{PML}]$ & Patrick M. Lashley & CASETEK & Holems!pat1@SUN.COM \\
\hline [PN23] & Peter Nellessen & SIEMENS & crtvax!pneSPICE.CS.CMU.EDU \\
\hline [PP 14] & Paul Pomes & UIUC & paul\%uxc@A.CS.UIUC.EDU \\
\hline$[\mathrm{PP} 36]$ & Paul Patton & HONEYWELL & --- none--- \\
\hline [PRT2 ] & Paul R. Taylor & $\begin{array}{l}\text { OSWEGO } \\
\text { rocksvax! }\end{array}$ & ! oswego! taylor@CS.ROCHESTER.EDU \\
\hline [PS 27 ] & Paal Spilling & NTA & SpillingeISI.EDU \\
\hline$[\mathrm{PSS} 1]$ & Phil S. Schwarz & DGPN & --- none--- \\
\hline [PW37] & Paul Woods & OSU & --- none--- \\
\hline [RA11] & Rick Adams & $\mathrm{CCI}$ & Rick@seismo.CSS.GOV \\
\hline [RA17] & Bob Albrightson & WASHINGTON & J BOB@WARD.CS.WASHINGTON.EDU \\
\hline [RA62 ] & Rex Aschenbrenner & $\begin{array}{l}\text { CGI } \\
\text { Rex } \div \text { CGIVB }\end{array}$ & 3CGI.CSNET@RELAY.CS.NET \\
\hline [RAJ3 ] & Richard Johnson & UCI-ICS & rajeICS.UCI.EDU \\
\hline [RAJ8 ] & Richard A. Jones & UColoB & jones@JVNCA.CSC.ORG \\
\hline [RAK12] & Richard A. Kawin & LLNL & kawin@MORDOR.S1.GOV \\
\hline [RAR2 2 ] & Robert A. Ridder & SYNTELNET & --- none--- \\
\hline [RAR2 3 ] & Richard A. Ragosa & $\mathrm{RCA}$ & --- none--- \\
\hline$[\mathrm{RB} 187]$ & Richard Baxter & YALE & baxter-richard@YALE. ARPA \\
\hline$[\mathrm{RB} 217]$ & Rafael Bracho & SPAR & RXB@KL.SRI.COM \\
\hline [RB218 ] & Randolph Bentson & $\begin{array}{l}\text { CSU } \\
\text { Bentson } \% \mathrm{C}\end{array}$ & Colostate.csnet@RELAY.CS.NET \\
\hline [RB219] & Robert Bybee & CHROMATICS & $5---$ none--- \\
\hline [RBB21 $]$ & Rick Blachley & SGI & --- none--- \\
\hline [RBN1] & Ronald Natalie, Jr. & BRL & ron@BRL.ARPA \\
\hline$[\mathrm{RBW}]$ & Richard B. Wales & UCLA & WALES@CS . UCLA. EDU \\
\hline [RC113 ] & Renee Collier & SAS & --- none--- \\
\hline [RCM9 ] & Robert C. McQueen & STEVENS & SIT.MCQUEEN@CU20B.COLUMBIA.EDU \\
\hline
\end{tabular}




\begin{tabular}{|c|c|c|c|}
\hline \multirow[t]{2}{*}[\mathrm{RD}80]{} & \multirow[t]{2}{*}{ Randal Dalhoff } & \multicolumn{2}{|c|}{ ISU } \\
\hline & & \multicolumn{2}{|c|}{ GR.RFCISUMVS.BITNET@WISCVM.WISC.EDU } \\
\hline [RD91] & Regine Dussaulx & CCVR & --- none--- \\
\hline [RDG12 ] & Robert D. Garvie & $\mathrm{CU}-\mathrm{COLO}$ & \\
\hline & & \multicolumn{2}{|c|}{ garvie\%grumpy. dnet@SPOT.COLORADO.EDU } \\
\hline [RDR4 ] & Dennis Rockwell & $\mathrm{BBN}$ & DRockwell@SH.CS.NET \\
\hline [RE22 ] & Rand Enas & $\mathrm{CDC}$ & CDC-DDN@DDN2 .ARPA \\
\hline [RER20 ] & Robert E. Rogers & CHRYSLER & --- none--- \\
\hline$[\mathrm{RF} 57]$ & Roger Fajman & $\mathrm{NIH}$ & rafonihcu.bitnet@WISCVM.WISC.EDU \\
\hline [RFD1 $]$ & Robert F. Donnelly & ARDC & rfd@ARDEC.ARPA \\
\hline [RG12] & Roger L. Gulbranson & UMINN & ROGERG@UMN-ACSS-UX.ARPA \\
\hline [RG92] & Richard Gopstein & $\mathrm{RCA}$ & Gopstein@RUTGERS.EDU \\
\hline [RH5 ] & Russell Hobby & UCDAVIS & rdhobby@UCDAVIS. UCDAVIS . EDU \\
\hline [RH6] & Robert Hinden & BBN & Hinden@CCV.BBN.COM \\
\hline [RH 60 ] & Roger Hale & MIT & Roger@LL-SST.ARPA \\
\hline [RHC3 ] & Robert H. Cole & UCL & robert@CS.UCL.AC.UK \\
\hline [RHS16] & Richard H. Sweed & RADC & SWEED@RADC-TOPS 20. ARPA \\
\hline [RJ59] & Ronald Johnson & APPLE & rljoapple.csnet@RELAY.CS.NET \\
\hline [RK51] & Richard Kisielewski & AIG & --- none--- \\
\hline [RKJ2 ] & Richard Johnsson & $\mathrm{DEC}$ & johnsson@DECWRL.DEC.COM \\
\hline [RKW6] & Robert K. Ware & CSM & --- none--- \\
\hline [RLB3 ] & Ronald L. Broersma & NOSC & Ron@NOSC.MIL \\
\hline [RLP 30$]$ & Ray L. Paulson & STPNET & --- none--- \\
\hline$[\mathrm{RLS} 6]$ & Ronald L. Smith & COINS & COINS@ISI.EDU \\
\hline [RM8 ] & Roy Marantz & RUTGERS & Marantz@RED.RUTGERS.EDU \\
\hline [RM120 ] & Richard McCarthy & \multicolumn{2}{|c|}{ BINGHAMTON } \\
\hline [RM125 ] & Ray McCorkle & NUWESNET & NSC-KEYPORT@DDN2.ARPA \\
\hline [RN25 ] & Roger Negaret & CNRS & --- none--- \\
\hline [RN29] & Ryo Nomura & $\mathrm{NTT}$ & \\
\hline & & \multicolumn{2}{|c|}{ nomura ${ }^{\circ} t t-20 @$ SUMEX-AIM.STANFORD. EDU } \\
\hline [RNM1 ] & Neil MacKenzie & RSRE & CLE $\%$ RSRE@CS.UCL.AC.UK \\
\hline [RP 8 8 ] & Russ Perry & CSUFRESNO & --- none--- \\
\hline$[\mathrm{RPP}]$ & Robert Pingree & NUSC & Pingree@NUSC.ARPA \\
\hline [RR2 ] & Raleigh Romine & TELEDYNE & romine@seismo.CSS.GOV \\
\hline [RR18 ] & Ron Reisor & UDEL & ron\%Vax3@LOUIE.UDEL.EDU \\
\hline [RR26] & William R. Reilly & USARMY & REILLY@COA. ARPA \\
\hline [RR97] & Robb Russell & DREXEL & \\
\hline & & \multicolumn{2}{|c|}{ ROBB $\% D U P R . B I T N E T @ W I S C V M . W I S C . E D U$} \\
\hline [RSD2 ] & Robert S. Dixon & \multicolumn{2}{|c|}{$\begin{array}{l}\text { OHIO } \\
\text { TSO258\%OHSTVMA.BITNETCWISCVM.WISC. EDU }\end{array}$} \\
\hline [RSM1 ] & Robert S. Miles & NRTC & RSM@NRTC.NORTHROP.COM \\
\hline$[\mathrm{RTL}]$ & Richard Lacoss & MITLL & Lacoss@XN.LL.MIT.EDU \\
\hline [RW101] & Randy Witlicki & witlickiowilliams. edu@RELAY.CS.NET & icki॰williams.edu@RELAY.CS.NET \\
\hline [RWH5 ] & Robert W. Henry & $\mathrm{UCB}$ & rwh@UCBVAX.Berkeley.EDU \\
\hline [RWT2 ] & Robert W. Tinker & DTNS & tinker@DTIX.ARPA \\
\hline$[\mathrm{SA}]$ & Scott Allen & GU & --- none--- \\
\hline
\end{tabular}




\begin{tabular}{|c|c|c|c|}
\hline [SA29] & Susan Ament & EMORY & OSSSA@EMORY.ARPA \\
\hline$[\mathrm{SAB} 17]$ & Scott A. Baird & FORMATIVE & --- none--- \\
\hline [SAK3 ] & Steven A. Kahn & JHAPL & Steve@APLVAX.ARPA \\
\hline [SB12] & Scott Bertilson & UMN & scott@UMN-REI-UC.ARPA \\
\hline [SB28 $]$ & Scott Bradner & HARVARD & sob@HARVARD . HARVARD. EDU \\
\hline [SB90] & Sean Brady & MACOM & brady@DCN9.ARPA \\
\hline [SB 98 ] & Stan Barber & BAYLOR & sob@BCM.TCM.EDU \\
\hline [SBW4 ] & Samuel Whidden & AMS & --- none--- \\
\hline$[\mathrm{SC} 54]$ & Scott Comer & ROSETTA & wert@RICE.EDU \\
\hline [SC59] & Stephen Campbell & $\begin{array}{l}\text { DARTMOUTH } \\
\text { steve } \% \text { dar }\end{array}$ & ctmouth. edu@RELAY.CS.NET \\
\hline$[\mathrm{SC} 81]$ & Sean Callaham & CIT & sean@ELXSI.CALTECH.EDU \\
\hline$[\mathrm{SD} 1]$ & Steve Dyer & $\mathrm{MMC}$ & dyer@HARVARD. HARVARD . EDU \\
\hline [SF 34 ] & Scott Fenstermacher & WMNET & \\
\hline & & scot & te $\%$ wmmvs.bitnet@WISCVM.WISC.EDU \\
\hline [SF 41$]$ & Steve Fogel & $\begin{array}{l}\text { MTCS } \\
\text { SFogel ! mt }\end{array}$ & Ecs!mtxinu@UCBARPA.Berkeley.EDU \\
\hline$[\mathrm{SFJ}]$ & Scott F. Johnston & CUBICOMP & --- none--- \\
\hline$[\mathrm{SGC}]$ & Steve Chipman & $\mathrm{BBN}$ & Chipman@F.BBN.COM \\
\hline$[\mathrm{SH} 37]$ & Sergio Heker & JVNC & heker@JVNCC.CSC.ORG \\
\hline [ SH4 7 ] & Steve Hallstrom & UW & \\
\hline \multicolumn{4}{|r|}{ steve\%uwacdc.bitnet@WISCVM.WISC.EDU } \\
\hline$[\mathrm{SH} 71]$ & Steve Herber & BGSU & rber\%andy.bgsu. edu@RELAY.CS.NET \\
\hline [SHB $]$ & Steven Blumenthal & BBN & BLUMENTHAL@VAX.BBN. COM \\
\hline [SI8] & Slawomir Ilnicki & $\mathrm{HP}$ & --- none--- \\
\hline$[\mathrm{SIP}]$ & Serge Polevitzky & SDSC & SERGE@NOSC-F 4.ARPA \\
\hline [SJS11] & Steven J. Schroeder & PENNSTATE & SJS $\%$ P SUVM.BITNET@WISCVM.WISC. EDU \\
\hline$[\mathrm{SL} 10]$ & Sandy Lerner & SPAR & sandy@SPAR-20.ARPA \\
\hline [SL55 ] & Sean Leaviseur & $\mathrm{UKC}$ & SJL。UKC.AC.UK@CS. UCL . AC.UK \\
\hline [SLH19] & Steven L. Howell & NSWCWO & --- none--- \\
\hline$[\mathrm{SM} 6]$ & Sean McLinden & DSL & McLinden@CADRE.DSL.PITTSBURGH.EDU \\
\hline [SM67 ] & Steve Miller & M2C & miller\%m2c.org@RELAY.CS.NET \\
\hline [SM96] & Scooter Morris & GENENTECH & scooter@CGL.UCSF.EDU \\
\hline [SMF 5 ] & Steven M. Feldman & TYMNET & \\
\hline \multicolumn{4}{|c|}{ hplabs!oliveb!tymix!feldman@UCBVAX.Berkeley.EDU } \\
\hline [SMK2 ] & Stephen M. King & HQEIS & KING@AFSC-HQ.ARPA \\
\hline [SMP 2 ] & Steven M. Polinsky & CUNY & \\
\hline & & & MPCUCUNYVM.BITNET@WISCVM.WISC.EDU \\
\hline [SMS1 $]$ & Steven M. Schultz & EATON & sms@ETM-WLV.EATON.COM \\
\hline [SS80 ] & Skip Schaller & UA & SKIP@SOLPL.AS.ARIZONA.EDU \\
\hline [SS110] & Stanfield Smith & NYTEL & stan\%gcylab.uucp@ITSGW. RP I. EDU \\
\hline$[\mathrm{ST} 13]$ & S. Takagi & ICOT & takagioicot.jp@RELAY.CS.NET \\
\hline$[\mathrm{SW7} 8]$ & Steve Wadle & EIKONIX & --- none--- \\
\hline$[S Y 8]$ & Shozo Yokota & FUJI & --- none--- \\
\hline [TA24] & Tohru Asami & $\mathrm{KDD}$ & --- none--- \\
\hline [TB4 $]$ & Ted Baker & FSU & tbaker@ISI.EDU \\
\hline [TB 64 ] & Tony Becker & $\mathrm{UCF}$ & tonyucf. edu@RELAY.CS.NET \\
\hline
\end{tabular}




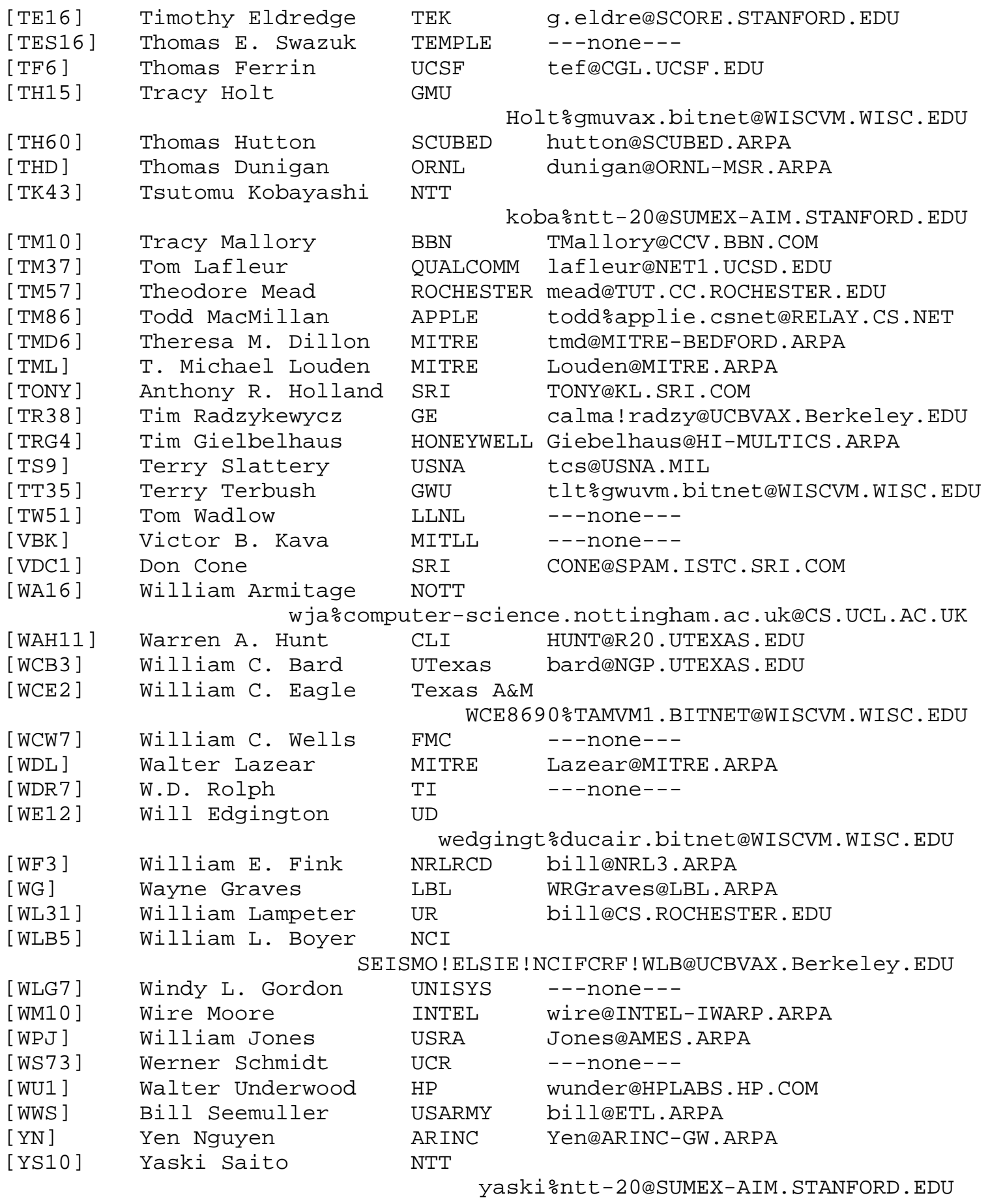


[YXD] Yves Despond

EPFL

[ZSU]

Zaw-Sing Su

despondoclsepf 51 .bitnet@WISCVM.WISC.EDU SRI ZSUQTSCA.ISTC.SRI.COM 


\section{APPENDIX A}

The network numbers in class A, B, and C network addresses are allocated among Research, Defense, Government (Non-Defense) and Commercial uses.

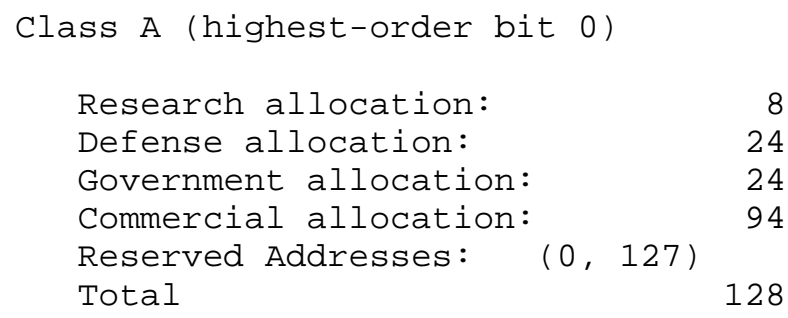

All addresses in this class are reserved for future use.

Experimental networks which later become operational need not be renumbered. Rather, the identifiers could be moved from Research to Defense, Government or Commercial status. Thus, network identifiers may change state among Research, Defense, Government and Commercial, but the number of identifiers allocated to each use must remain within the limits indicated above. To make possible this fluid assignment, the network identifier spaces are not allocated by simple partition, but 
rather by specific assignment.

Also, organizations not currently affiliated with the Internet may be assigned numbers for networks for non-connected service. If at some later time such networks are connected to the Internet (with appropriate prermissions and approvals) the networks need not be renumbered. 